\title{
THE THERMAL CONDUCTIVITY OF HIGH PURITY VANADIUM
}

Wayne Douglas Jung

Ph. D. Thesis Submitted to Iowa State University

\author{
Ames Laboratory, ERDA \\ Iowa State University \\ Ames, Iowa 50011
}

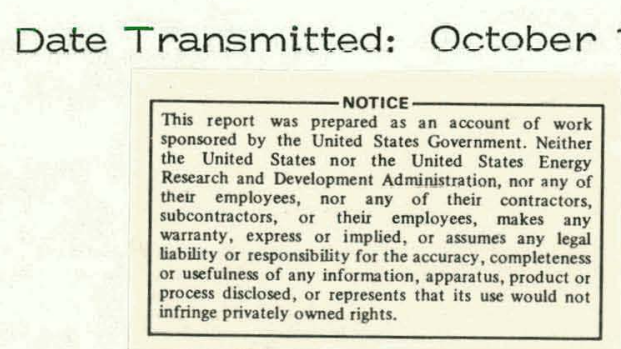

PREPARED FOR THE U.S. ENERGY RESEARCH AND DEVELOPMENT ADMINISTRATION UNDER CONTRACT NO. W-7405-eng-82 


\section{DISCLAIMER}

This report was prepared as an account of work sponsored by an agency of the United States Government. Neither the United States Government nor any agency Thereof, nor any of their employees, makes any warranty, express or implied, or assumes any legal liability or responsibility for the accuracy, completeness, or usefulness of any information, apparatus, product, or process disclosed, or represents that its use would not infringe privately owned rights. Reference herein to any specific commercial product, process, or service by trade name, trademark, manufacturer, or otherwise does not necessarily constitute or imply its endorsement, recommendation, or favoring by the United States Government or any agency thereof. The views and opinions of authors expressed herein do not necessarily state or reflect those of the United States Government or any agency thereof. 


\section{DISCLAIMER}

Portions of this document may be illegible in electronic image products. Images are produced from the best available original document. 
This report was prepared as an account of work sponsored by the United States Government. Neither the United States nor the United States Energy Research and Development Administration, nor any. of their employees, nor any of their contractors, subcontractors, or their employees, makes any warranty, express or implied, or assumes any legal liability or responsibility for the accuracy, completeness, or usefulness of any information, apparatus, product or process disclosed, or represents that its use would not inf ringe privately owned rights.

Available from: National Terhnical Information Service U. S. Department of Commerce P.O. Box 1553

Springfield, VA 22161

Price: Microfiche $\$ 2.25$ 


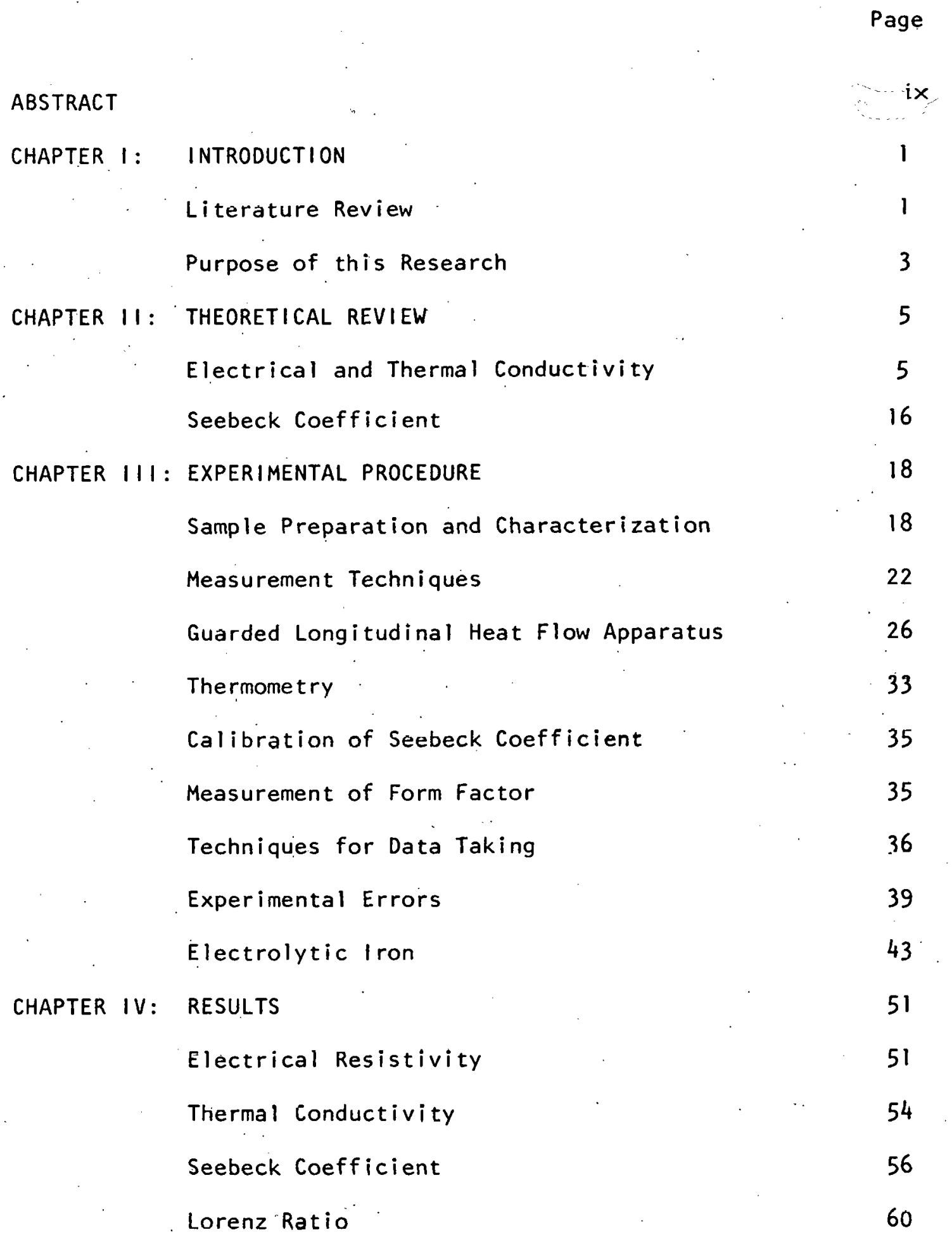


CHAPTER V: DISCUSSION . . $\quad 62$

Thermal Conductivity 62

Electrical Resistivity 66

$\begin{array}{ll}\text { Seebeck Coefficient } & 76\end{array}$

CHAPTER VI: SUMMARY. $\quad 80$

LITERATURE CITED

ACKNOWLEDGMENTS $\quad . \quad 85$

APPENDIX A: SHIELD DIFFERENTIAL TEMPERATURE CONTROLLER 86

APPEND IX B: A. C. RES ISTANCE TEMPERATURE CONTROLLER 89

APPENDIX C: THERMOCOUPLE CALIBRATION AND ERROR ANALYSIS 
Table 1. Sample characteristics 19

Table 2. SSMS analysis of sample $1 \quad 21$

Table 3. Characteristics of sample SRM 734

Table 4 . The coefficients $A$ and $B$ of the thermal
resistivity of vanadium

Table 5. Uncertainty in absolute temperature measurement

Table 6. Uncertainty in the thermocouple Seebeck coefficient and temperature gradient measurement 
Figure 1. The four probe electrical resistivity and thermal conductivity technique.

Figure 2. Sample holder wiring diagram.

Figure 3. Thermal conductivity sample holder.

Figure 4. The thermal conductivity of electrolytic iron as a function of temperature.

Figure 5. The electrical resistivity of electrolytic iron as a function of temperature.

Figure 6. The Seebeck coefficient of electrolytic iron as a function of temperature.

Figure 7. Comparison of the thermal conductivity, electrical resistivity and Seebeck coefficient of electrolytic iron to the results of NBS.

Figure 8. The electrical resistivity of vanadium as a function of temperature for four samples of high purity vanadium. The lowest purity. sample, sample l, had an electrical resistivity which was affected by impurities over the entire temperature range.

Figure 9. The log of the ideal electrical resistivity plotted as a function of the log of the temperature. Vanadium had an ldẹal electrical resistivity which varied as $\mathrm{T}^{3.4}$ from $10 \mathrm{~K}$ to $50 \mathrm{~K}$.

Figure 10. The thermal conductivity of vanadium as a function of temperature. The lowest purity sample, sample l, had a thermal conductivity which was limited by impurley scattering over the entire temperature range.

Figure 11. The log of the intrinsic thermal resistivity as a function of the log of the temperature. The intrinsic thermal resistivity varied as $\mathrm{T}^{2}$ from $10 \mathrm{~K}$ to $25 \mathrm{~K}$. 
Figure 12. The Seebeck coefficient of vanadium as a function of temperature. The Seebeck coefficient varied with sample purity at low temperatures and was positive for temperatures less than $240 \mathrm{~K}$. Above $240 \mathrm{~K}$ the Seebeck coefficient was negative.

Figure 13. The Lorenz ratio of vanadium plotted as a function of temperature. The Lorenz ratio of vanadium was less than $L_{0}$ over the entire temperature range.

Figure 14. The thermal resistlvity of vanadium times temperature vs $T^{3}$. The linear dependence shows that: the intrinsic thermal resistiv$i$ ty of vanadium had a $\mathrm{T}^{2}$ temperature dependence at low temperatures.

Figure 15. $\rho_{i}(T) / \rho_{B}(T)$ vs $T$ for vanadium, where $\rho_{B}(T)$ is the Block formula. Above $70 \mathrm{~K} \rho_{i}(T) / \rho_{B}(T)$ was constant, thus the intrinsic thermal resistivity of vanadium had the temperature dependence predicted by the Block formula.

Figure 16. $\rho_{i}(T) / T^{3}$ vs $T^{2}$ for vanadium. $\rho_{i}(T) / T^{3}$ varied linearly with temperature at low temperatures. The large intcrcepts show that at low temperature the intrinsic thermal resistivity of vanadium had a $T^{3}$ term.

Figure 17. $\rho_{i}(T) / T^{2}$ vs $T^{3}$ for vanadium. The $T^{3}=0$ intercept is non-zero showing that the intrinsic electrical resistivity of vanadium may have a $T^{2}$ term at low temperatures.

Figure 18. $W_{i}(T) / T$ vs $T$ for vanadium. The $T=0$ intercept

is zero. Hence vanadium showed no evidence of low temperature electron-electron scattering.

Figure 19. The Seebeck coefficient of vanadium plotted as TS $(T)$ vs $T^{2}$.

Figure 20. The Seebeck coefficient of vanadium plotted as TS (T) vs T. The linear slope showes that at high temperatures the Seebeck coefficient varied as $\alpha^{\prime}+\beta^{\prime} / T$. 
Figure 21. D.C. proportional temperature controller.

Figure 22. A.C. resistance temperature controller bridge circuit.

Figure 23. Block diagram of the a.c. temperature controller.

Figure 24. The d.c. amplifier circuit. 


\section{ABSTRACT}

The thermal conductivity, Seebeck coefficient and electrical resistivity of four high purity vanadium samples have been measured as functions of temperature over the temperature range $5 \mathrm{~K}$ to $300 \mathrm{~K}$. The highest purity sample had a resistance ratio $\left(\rho_{273 k} / \rho_{4.2} k\right)$ of 1524. The highest purity sample had a thermal conductivity maximum of $920 \mathrm{~W} / \mathrm{mK}$ at $9 \mathrm{~K}$ and had a thermal conductivity of $35 \mathrm{~W} / \mathrm{mK}$ at room temperature. At low temperatures the thermal resistivity was limited by the scattering of electrons by impurities and phonons. The thermal resistivity of vanadium departed from Mathiessen's rule at low temperatures. The electrical resistivity and Seebeck coefficient of high purity vanadium showed no anomalous behavior above $130 \mathrm{~K}$. The intrinsic electrical resistivity at low temperatures was due primarily to interband scattering of electrons. The Seebeck coefficient was positive from $10 \mathrm{~K}$ to $240 \mathrm{~K}$ and had a maximum which was dependent upon sample purity. 


\section{CHAPTER I: INTRODUCT ION}

\section{Literature Review}

Vanadium is a transition metal which is widely distributed over the earth's crust. It is somewhat scarce and it is difficult to purify. Vanadium was first discovered by $M$. del Rio in 1801; however, his discovery was misinterpreted as impure chromium. Later in 1830, vanadium was re-discovered by N. G. Sefstron who named the metal after the Swedish goddess Vanadis because of its multicolored compounds (1). Commercial vanadium is purified today by the calcium reduction of $\mathrm{V}_{2} \mathrm{O}_{5}(2,3)$, however the interstitial impurity content of this quality metal is still quite high. Carlson and Owen (4) have produced vanadium samples of greater than $99.9 \%$ purity by reducing $\mathrm{V}_{2} \mathrm{O}_{5}$ with aluminum and Sullivan. (5) and Lel and Sullivan (6) have purified vanadium to $99.99 \%$ purity by the fused-salt electrorefining technique at the U.S. Bureau of Mines. Vanadium has been further purified by Reed (7) with the electronbeam float-zone refining method and by Carlson et al. (8) who produced vanadium samples of less than 5 wt ppm interstitial impurity content with the electrotransport technique.

Vanadium is a b.c.c. transition metal (1). It is a type 11 superconductor with a transition temperature that varies somewhat with purity (9-12). Radebaugh and Keesom (12) have reported from specific heat measurements that the superconducting transition temperature of high purity vanadium is, $5.379 \pm 0.004 \mathrm{~K}$. Radebaugh also reported that vanadium has a Debye temperature of $382 \pm 10 \mathrm{~K}$ at $0 \mathrm{~K}$. The Debye 
temperature calculated by Radebaugh is somewhat different from the Debye temperature calculated from elastic constants measurements $(13,14)$ and earlier specific heat measurements $(9,10)$.

Various anomalies have been reported in the temperature dependence of a number of the physical properties of vanadium occurring between $180 \mathrm{~K}$ and 250K; electrical resistivity $(15-20)$, thermal expansion $(13,16,18,21)$, magnetic susceptibility $(15,22)$ and Seebeck coefficient (23). Westlake $(24,25)$ has shown that hydrogen affects the electrical resistivity of vanadium above 150K. Westlake reported that below a critical temperature $T_{c}$, hydrogen is no longer soluble in vanadium and that up to 3.5 at. percent hydrogen can precipitate in the lattice below $T_{c}$. The critical temperature $T_{c}$ varies between $180 \mathrm{~K}$ and $250 \mathrm{~K}$ and increases with temperature as the hydrogen concentration increases. Chiba and Takano (26) studied the hydrogen precipitation in vanadium with electron microscopy. Chiba reported that hydrogen precipitates into two superstructures: a cubic superstructure and a tetragonal superstructure. The cubic superstructure was reported to occur on fast cooling and the tetragonal structure occurs on slow cooling in samples which have low additional interstitial impurities. Vanadium samples which had high additional impurities were found to have a pseudo memory affect. The hydrogen precipitated in the same superstructure and the same location in the sample after suecessive heating and cooling.

The Seebeck coefficient of vanadium has been measured by Mackintosh and Sill (23) and an anomaly was observed at $218 \mathrm{~K}$. In addition the 
Seebeck coefficient was reported to have a temperature dependent hysteresis between $125 \mathrm{~K}$ and $300 \mathrm{~K}$ (the highest temperature measured). Mackintosh suggested that a structural phase change was occurring at $218 \mathrm{~K}$ and that further measurements should be taken on samples of higher purity.

The electrical resistivity of vanadium has been measured by several investigators $(15-20)$ on samples of varying impurity concentration and most authors have reported anomalous behavior between $180 \mathrm{~K}$ to $250 K$.

Rosenberg (27) and White and Woods (19) have measured the thermal conductivity of vanadium below 100K. No anomalous behavior was reported in the thermal conductivity. However, the samples were of low purity and no low temperature thermal conductivity maximum was observed.

\section{Purpose of this Research}

The purpose of this research was to measure the electrical resistivity, thermal conductivity and Seebeck coefficient of high purity vanadium. High purity samples were needed in order to investigate the intrinsic electron scattering mechanisms of vanadium. The results were compared to theoretical models of the transport properties of metals.

Earlier measurements of the transport properties of vanadium were made on samples of low purity and the electrical resistivity and. Seebeck coefficient behaved anomalously between $140 \mathrm{~K}$ to $250 \mathrm{~K}$. In this research, high purity samples were measured to test whether the 
anomalous behavior of vanadium was an intrinsic property of vanadium or whether the anomalous behavior was due to impurities. 


\section{CHAPTER II. THEORETICAL REVIEW}

\section{Electrical and Thermal Conductivity}

The thermal conductivity of a metal is the conduction of energy by electrons and phonons. The total thermal conductivity of a metal can be written as,

$$
\lambda(T)=\lambda_{e}(T)+\lambda_{g}(T)
$$

where $\lambda(T)$ is the total thermal conductivity, $\lambda_{e}(T)$ is the electronic thermal conductivity and $\lambda_{g}(T)$ is the phonon thermal conductivity. In pure elemental metals the electronic thermal conductivity is greater than the phonon thermal conductivity and the separation of the total thermal conductivity into phonon and electronic components is difficult (28). In this theoretical discussion, the total thermal conductivity will be treated as if it were due entirely to conduction by the electrons. The electrical conductivity of a metal $(\sigma)$ is the conduction of charge by electrons. The electrical resistivity of a metal $(1 / \sigma)$ can bc scparated into components,

$$
\rho(T)=\rho_{0}+\rho_{i}(T)
$$

where $\rho(T)$ is the total electrical resistivity, $\rho_{0}$ is the residual electrical resistivity and $\rho_{i}(T)$ is the intrinsic electrical resistivity. Equation 2 is Mathiessen's rule. The residual electrical resistivity is temperature independent and, for a given metal, depends only upon sample purity. The intrinsic electrical resistivity is an intrinsic 
property of a metal and varies with temperature.

The Lorenz ratio of a metal is

$$
L(T)=\frac{\lambda(T)}{\sigma(T) T}
$$

At low temperatures and at high temperatures the Lorenz ratio is a constant and has the theoretically calculated value

$$
L_{o}=\frac{\pi^{2}}{3}\left(\frac{K_{B}}{e}\right)^{2}=2.45 \times 10^{-8} v^{2} / K^{2}
$$

where $K_{B}$ is the Boltzmann constant and $e$ is the electronic charge.

The relationship

$$
\frac{\lambda}{\sigma T}=L_{0}
$$

is the Wiedemann-Franz-Lorenz (WFL) rule. It holds for most metals at low and high temperatures. However at intermediate temperatures the WFL rule breaks down.

The transport of energy and electrical charge by electrons is limited by the scattering of electrons by phonons and ionized impurities. The transport properties of metals has been reviewed by Klemens $(28-30)$, Mendelson and Rosenberg (31), Ziman (32), Wilson (33), Peierls (34) and others.

When a metal is at equilibrium the electrons are distributed in momentum space in an equilibrium distribution $f^{\circ}(\underline{k})$ where $f^{\circ}(\underline{k})$ is the 
Fermi-Dirac distribution.

$$
f^{O}(\underline{k})=\left[1+\exp \left\{\left(E(\underline{k})-E_{F}\right) / K_{B} T\right\}\right]^{-1}
$$

where $E(\underline{k})$ is the electron energy and $E_{F}$ is the Fermi energy. A temperature gradient $\nabla T$ or an electric field $\underline{F}$ perturbs the electron distribution from equilibrium. The electrons encounter collisions which tend to restore the perturbed electron distribution to equilibrium with a characteristic relaxation time $\tau(k)$. Consequently, a steady state situation develops which is described by the Boltzmann equation

$$
\left.\frac{e}{h} \underline{F} \cdot \frac{\partial f(\underline{k})}{\partial \underline{k}}+\underline{v} \cdot \nabla T \frac{\partial f(\underline{k})}{\partial T}=\frac{d f(\underline{k})}{d t}\right]_{c} .
$$

The first term on the left of equation 7 is the perturbation caused by the electric field and the second term is the perturbation caused by the temperature gradient. The collision term on the right of equation 7 is expressed in the relaxation approximation as,

$$
\begin{aligned}
\left.\frac{d f(\underline{k})}{d t}\right]_{c} & =\frac{f^{\circ}(\underline{k})-f(\underline{k})}{\tau(\underline{k})} \\
& =-\frac{g(\underline{k})}{\tau(\underline{k})}
\end{aligned}
$$

where $g(\underline{k})$ is the deviation of the electron distribution from equilibrium, and $\tau(\underline{k})$ is the relaxation time.

The electrical current density $J$ is, 


$$
\underline{J}=\frac{2}{(2 \pi)} 3 \int \text { e } \underline{v}(\underline{k}) g(\underline{k}) d \underline{k}
$$

The heat current density $\underline{Q}$ is,

$$
\begin{gathered}
\underline{Q}=\frac{2}{(2 \pi)^{3}} \int\left[E(\underline{k})-E_{F}\right] \underline{v}(\underline{k}) g(\underline{k}) d \underline{k} . \\
\text { all } \underline{k} \text { space }
\end{gathered}
$$

The electrical conductivity is defined by Ohm's law,

$$
\underline{J}=\sigma F
$$

and is calculated from equation 9 and 8 when $\nabla T$ is zero (30). For an isotropic (cubic) metal

$$
\sigma=\frac{e^{2}}{12 \pi^{3}} \int \frac{\tau_{e}(\underline{k}) v^{2} \cdot d G}{\left|\nabla_{k} E(\underline{k})\right|}
$$

where $d G$ is an integration over the area of the Fermi surface and $v$ is the electron velocity at the Fermi energy.

The thermal conductivity is defined by

$$
\underline{Q}=-\lambda \nabla T
$$

and is calculated from equation 10 and 8 when the current density, $\mathrm{J}$, is zero. For an isotropic metal at low temperatures $\left(K_{B} T \ll E_{F}\right)$ the thermal conductivity is (30) 


$$
\lambda=\frac{K_{B} T}{36 \pi} \int \frac{\tau_{\lambda}(\underline{k}) v^{2} \cdot d G}{\left|\nabla_{k} \bar{\varepsilon}(\underline{k})\right|} .
$$

The WFL rule, equation 5 , holds if the relaxation times for electrical ( $\left.\tau_{e}\right)$ and thermal $\left(\tau_{\lambda}\right)$ conduction are equal. The electronic and thermal relaxation times are equal at low temperatures where impurity scattering dominates and at high temperatures where nearly elastic electron-phonon scattering dominates. At intermediate temperatures the WFL rule breaks down owing to inelastic electron-phonon scattering. Klemens $(28-30,35)$ discusses the difference in relaxation times for electrical and thermal conduction. An electric field $\underline{F}$ perturbs the electron distribution such that,

$$
g(\underline{k}) \propto \underline{k} \cdot \underline{F} \frac{d f^{0}}{d \varepsilon}
$$

where

$$
\varepsilon=E(\underline{k})-E_{F} .
$$

The deviation $g(\underline{k})$ of the electron distribution for electrical conduction is equivalent to a displacement of the Fermi surface in $\underline{k}$ space. One side of the Fermi surface has an excess number of electrons while the 
other side has a deficiency of electrons. Consequently a scattering process can restore equilibrium by moving an electron from the excess side of the Fermi surface to the deficient side. Hence large angle scattering is more effective in restoring equilibrium than small angle scattering. The effective relaxation time for electrical conduction is (28)

$$
\frac{1}{\tau_{e}}=\frac{1}{\tau_{0}}(1-\bar{\mu})
$$

where $\tau_{0}$ is the mean free time of an electron between collisions and $\bar{\mu}$ is the average cosine of the scattering angle.

A temperature gradient $\nabla T$ perturbs the electron distribution such that

$$
g(\underline{k}) \propto \underline{k} \cdot \nabla T \varepsilon \frac{d f^{O}}{d \varepsilon} .
$$

The deviation $g(\underline{k})$ for thermal conduction is equivalent to increasing the electron energy on one side of the Fermi surface and decreasing it on the opposite side. There are two ways in which scattering can restore equilibrium; scattering processes which change the direction of the electron but conserve the electron energy (elastic scattering) and scattering which changes the electron energy but not necessarily 
the electron's direction (inelastic scattering). Klemens called the elastic scattering process "horizontal" and the inelastic scattering process "vertical". Most electron-phonon scattering processes are inelastic at low temperatures. For horizontal scattering the effective thermal relaxation time is the relaxation time discussed in equation 17 , and the WFL rule holds. For vertical processes the effective relaxation time is $1 / \tau_{0}$, and the WFL rule breaks down (28).

At temperatures much less than the Debye temperature of a metal, the phonons are of long wavelength and an electron-phonon interaction causes little change to the electron momentum or energy. Thus at low temperatures the electron-phonon interaction is ineffective in restoring the perturbed electron distribution to equilibrium and consequently the thermal and electrical conductivity of a pure metal increase without bound as the temperature approaches zero. The electron distribution is restored to equilibrium by ionized impurities and defects in the crystal lattice. At low temperatures the electron mean free path, $\ell$, where,

$$
l=v(\underline{k}) \tau(\underline{k})
$$

is dependent upon the concentration of impurities and defects in the lattice and is independent of temperature. Thus the electrical 
conductivity of metal at low temperature is (32)

$$
\sigma=\frac{e^{2}}{12 \pi^{3} \hbar} \int \ell d G
$$

and $i t$ is independent of temperature for impurity scattering.

The thermal conductivity of a metal is also limited by impurity scattering and is

$$
\lambda=\frac{K_{B}^{2} T}{36 \pi \hbar} \int l d G
$$

At low temperatures, when impurity electron scattering dominates, the thermal conductivity of a metal varies linearly with temperature, and the WFL rule is valid.

The scattering of electrons by phonons becomes important as the temperature is increased and the phonons populate to high frequencies. The general form of an electron phonon interaction is,

$$
\underline{k} \pm \underline{q}+\underline{k}^{\prime}+\underline{B}
$$


where $\underline{k}$ is the initial electron wavevector, $q$ is a phonon (either emitted or absorbed), $\underline{k}^{\prime}$ is the final electron wavevector and $\underline{B}$ is a reciprocal lattice vector. Normal scattering of electrons occurs when $\underline{B}=0$ (electron-phonon momentum is conserved) and Umklapp scattering or $U$-processes occur when $\underline{B} \neq 0$ (electron-phonon momentum is not conserved). The normal scattering of phonons occurs at all temperatures, however as shown by Peierls $(34)$; U-processes occur when the phonon wavevector is greater than or equal to $q^{\prime}$. wherc

$$
q^{\prime}=8-2 k_{F}
$$

and $k_{F}$ is the electron Fermi wavevector. Consequently U-processes occur at temperatures sufficiently high where phonons are populated to wavevectors equal to or greater than $q^{\prime}$. Klemens and Jackson (36) have shown that U-processes occur at low temperatures in metals which have Fermi surfaces which touch the Brillouin zone boundary. U-processes are an important scattering mechanism since they move an electron through a large angle across the Fermi surface in a single step (28).

The intrinsic electrical resistivity. of a metal was first solved by Bloch (37) and the calculation has been reviewed by Wilson (33), Jones (38), Ziman (32) and others. The transport equation for electrical resistivity has also been solved by a variational technique discussed by Kohler $(39,40)$ and Sondheimer (41). 
The assumptions of the Bloch model are, normal electron-phonon scattering, a Debye phonon spectrum, a spherical Fermi surface and electric conduction in one parabolic band. Bloch showed that

$$
\rho_{i}(T)=4 \rho_{\theta}\left(\frac{T}{\theta}\right)^{5} J_{5}\left(\frac{\theta}{T}\right)
$$

where $\rho_{\theta}$ is a constant, $\theta$ is the Debye characteristic temperature and $J_{5}\left(\frac{\theta}{T}\right)$ is the Debye integral for $N=5$.

$$
J_{N}\left(\frac{\theta}{T}\right)=\int_{0}^{\theta / T} \frac{e^{z} z^{N} d z}{\left(e^{z}-1\right)^{2}}
$$

At low temperatures $(T<\theta / 20) J_{5}(\theta / T)$ is a constant and

$$
\rho_{i}(T)=497.6 \cdot \rho_{\theta}\left(\frac{T}{\theta}\right)^{5}
$$

At high temperatures $(T>\theta) J_{5}(\theta / T)$ varies as $1 / 4(\theta / T)^{4}$. Thus at high temperatures

$$
\rho_{i}(T)=\rho_{\theta}(T / \theta)
$$

The intrinsic thermal resistivity $W_{i}(T)$ of a metal was derived by Wilson (42) with the same assumptions made by Bloch. Wilson showed that,

$$
\begin{aligned}
& W_{i}(T)=1 / \lambda_{i}(T) \\
& =\frac{4 \rho_{\theta}}{L_{0}^{T}}\left(\frac{T}{\theta}\right)^{5}\left[\left\{1+\frac{3}{4 \pi^{2}}\left(\frac{2}{N_{a}}\right)^{2 / 3}\left(\frac{\theta}{T}\right)^{2}\right\} J_{5}\left(\frac{\theta}{T}\right)-\frac{1}{2 \pi^{2}} J_{7}\left(\frac{\theta}{T}\right)\right]
\end{aligned}
$$

where $N_{a}$ is the number of conduction electrons per atom. The first term 
in equation 28 is the thermal resistance due to horizontal electron scattering, the second term is the thermal resistance due to vertical electron scattering and the third term is a correlation term since vertical and horizontal scattering do not act independently. At low temperatures $(T<\theta / 20)$ the second term in equation 28 dominates and

$$
w_{i}(T) \propto T^{2}
$$

At high temperature $(T>\theta)$ the first term dominates and

$$
W_{i}(T)=\text { constant }
$$

At intermediate temperatures the Wilson theory predicts a minimum in the thermal resistivity at $\theta / 5$. Ziman (43) has shown that the minimum is caused by the neglect of U-processes. U-processes tend to place more emphasis on horizontal scattering and raise the intrinsic thermal resistivity at high and intermediate temperatures.

The addition of $U$-processes to the electrical resistivity and thermal resistivity is complicated because U-processes depend upon the detailed shape of the Fermi surface. Ziman (43) has shown that U-processes increase the electrical resistivity at high and intermediate temperatures above the value predicted by the Bloch model.

$$
\rho_{i}(T)=\rho_{B}(T)+\rho_{U}(T)
$$

where $\rho_{B}(T)$ is the Bloch electrical resistivity and $\rho_{U}(T)$ is an additional resistivity caused by U-processes. As discussed earlier, 
U-processes can occur when the phonon momentum is greater than $q^{\prime}$ where $q^{\prime}$ is defined in equation 23. Thus $Z$ iman (32) has suggested that

$$
\rho_{u}(T) \propto \exp (\theta / B T)
$$

where

$$
B=D / q^{\prime}
$$

$D$ is the Debye radius. However, Ziman (32) has pointed out that an exponential behavior of $\rho_{u}(T)$ would be difficult to observe.

\section{Seebeck Coefficient}

The Seebeck coefficient of a metal can be written as the sum of two terms (44).

$$
S(T)=S_{d}(T)+S_{g}(T)
$$

where $S_{d}(T)$ is the diffusion Seebeck coefficient due to the diffusion of electrons in a temperature gradient and $S_{g}(T)$ is the phonon-drag Seebeck coefficient. The phonon drag term is a second order effect due to the perturbation of the phonon distribution in a temperature gradient.

The diffusion Seebeck coefficient can be calculated from the transport equations 9 and 10 . The Seebeck coefficient is related to the Peltier coefficient $(\pi)$ by the Kelvin relation,

$$
S=\pi / T \text {. }
$$

The Peltier coefficient is defined by, 


$$
\pi=Q / J \text { when } \nabla T=0 .
$$

Thus the diffusion Seebeck coefficient is (32) for $K_{B} T \ll E_{F}$,

$$
S_{d}(T)=\frac{\pi^{2} \cdot \frac{K_{B}{ }^{2} T}{3}}{e}\left[\frac{\partial \ln \sigma(E)}{\partial E}\right]_{E=E_{F}}
$$

where $\sigma(E)$ is the electrical conductivity calculated for electrons of energy $E$. The electrical conductivity can be expressed in terms of the electron mean free path $\ell$ equation 20 , thus (32)

$$
S_{d}(T)=\frac{\pi^{2}}{3} \frac{K_{B}{ }^{2} T}{e}\left[\frac{\partial \ln \ell}{\partial E}+\frac{\partial \ln G}{\partial E}\right]_{E=E_{F}}
$$

where.G is the area of the Fermi surface. The sign of the diffusion Seebeck coefficient depends upon the sign of the second term in equation 38 (32).

The derivative of the Fermi surface with respect to energy can be of either sign depending upon whether or not the Fermi surface is extending beyond the Rrillouin zone boundary.

For most metals, at low temperatures, both terms in equation 38 are independent of temperature (44) and

$$
S_{d}(T) \propto T .
$$




\section{CHAPTER 111. EXPERIMENTAL PROCEDURE}

\section{Sample Preparation and Characterization}

The four vanadium samples measured in this research were prepared at the Ames Laboratory by F. A. Schmidt by the electrotransport technique. The details of the apparatus have been discussed by Peterson and Schmidt (45) and Peterson et al (46). Samples are purified by electrotransport by passing large electrical currents through cylindrical samples, causing resistive heating and impurity migration. Prior to electrotransport the samples are heated to $1400^{\circ} \mathrm{C}$ in a vacuum of better than $5 \times 10^{-10}$ Torr in order to drive off gaseous impurities. The sample chamber is then sealed and pressurized with purified helium gas to a pressure of $13 \mathrm{~cm}$. of $\mathrm{Hg}$. A current density of $2150 \cdot \mathrm{A} / \mathrm{cm}^{2}$ is passed through the sample for 210 to $220 \mathrm{~h}$. The high current density heats the sample to $1650^{\circ} \mathrm{C}$ and causes impurity migration. The helium pressure reduces the sublimation rate of vanadium. The electrotransport purification process is useful in reducing the nonmetallic elements $c, 0$ and $N$. The high temperature degassing prior to electrotransport reduces the hydrogen concentration to less than 1 wt. ppm. 'Several impurities, notably silicon, do not migrate well during electrotransport (8). Consequently it is necessary to electrotransport a base material which is as pure as possible. The starting material for this investigation was double-electrorefined vanadium from the U.S. Bureau of Mines at

ISchmidt, F. A., Ames Laboratory, Ames, lowa, Private Communication. 
Boulder City.

Four samples of vanadium were measured in this research. The samples were cylindrical with an average diameter of $0.25 \mathrm{~cm}$ and lengths varying from $2.5 \mathrm{~cm}$ to $4 \mathrm{~cm}$. The sample characteristics are listed in Table 1. All four samples were polycrystaline.

Table 1. Sample characteristics

\begin{tabular}{cccccc}
\hline Sample & $\begin{array}{c}\text { Length } \\
(\mathrm{cm})\end{array}$ & $\begin{array}{c}\text { Diameter } \begin{array}{c}\rho 273 \mathrm{~K} \\
(\mathrm{~cm})\end{array} \\
(\mu \Omega \mathrm{cm})\end{array}$ & $\begin{array}{c}\text { Resistance } \\
\text { Ratio } \\
\rho 273 \mathrm{~K} / 04.2 \mathrm{~K}\end{array}$ & $\begin{array}{c}\text { Impurity Concentrationa } \\
0+\mathrm{C}+\mathrm{N} \\
(\mathrm{at} . \mathrm{ppm})\end{array}$ \\
\hline 1 & 2.5 & 0.263 & 19.61 & 37.6 & $1230^{\mathrm{b}}$ \\
2 & 3.97 & 0.260 & 18.72 & 81.5 & 570 \\
3 & 3.65 & 0.205 & 18.69 & 785 & 55 \\
4 & 4.3 & 0.241 & 18.90 & 1524 & 28 \\
\hline
\end{tabular}

${ }^{a}$ Estimates based on resistance ratio from Carlson (1). The resistance ratios reported above were corrected to $\rho_{298} / \rho_{4.2}$ for comparison with ( 1 ).

b. Impurity concentration is an extrapolation of the results of Carlson (1).

At low temperatures the electrical resistivity of a metal is constant and results from the scattering of electrons by impurities. At room temperature the electrical resistivity of a pure metal is due to the scattering of electrons by phonons. Thus the ratio of the 
electrical resistivity of a metal at $273 \mathrm{~K}$ to the electrical resistivity at $4.2 \mathrm{~K}\left(\rho_{273 K^{/ \rho}} \rho_{4.2 K}\right)$ is a convenient measure of the purity of a metal. The samples measured in this research had residual resistance ratios (RRR) that varied from 38 to 1524 .

The major impurities present in vanadium affecting the low temperature electrical resistivity are $0, N, C$ and $S i$. The Si concentration of the samples was low due to the high purlty of the base material that was electrotransported. $\mathrm{O}, \mathrm{N}$ and $\mathrm{C}$ impurities were reduced by the electrotransport. Reed (7) and Carlson et al. (8) have analyzed the impurity content of $\mathrm{O}, \mathrm{N}$ and $\mathrm{C}$ as a function of RRR. The impurity. concentration results of Reed were slightly higher than those of Carlson. Carlson found that the total impurity concentration of $\mathrm{C}+\mathrm{O}+\mathrm{N}$ was proportional to the inverse of the resistance ratio. By comparing the resistance ratio of the four samples measured in this research with the results of Carlson, the total impurity concentration of $0+N+C$ was estimated. The results of this calculation are listed in Table 1.

The lowest purity sample, sample 1, was analyzed by spark source mass spectrometry (SSMS) for impurities by R. Conzemius at Ames Laboratory. Prior to SSMS the sample was electropolished. SSMS is sensitive to surface contamination and consequently is a poor method for measuring 0 and $C$. The SSMS analysis was conducted primarily to measure the impurity concentration of other metals. The results of the analysis are listed in Table 2. The predominant impurities in Sample 1 in at. ppm are: $\mathrm{Cl}-30, \mathrm{~W}-23, \mathrm{Cu}-22, \mathrm{Cr}-10$ and $\mathrm{Fe}-5$. The Si concentration was 
Table 2. SSMS Analysis of Sample $1^{a, b}$

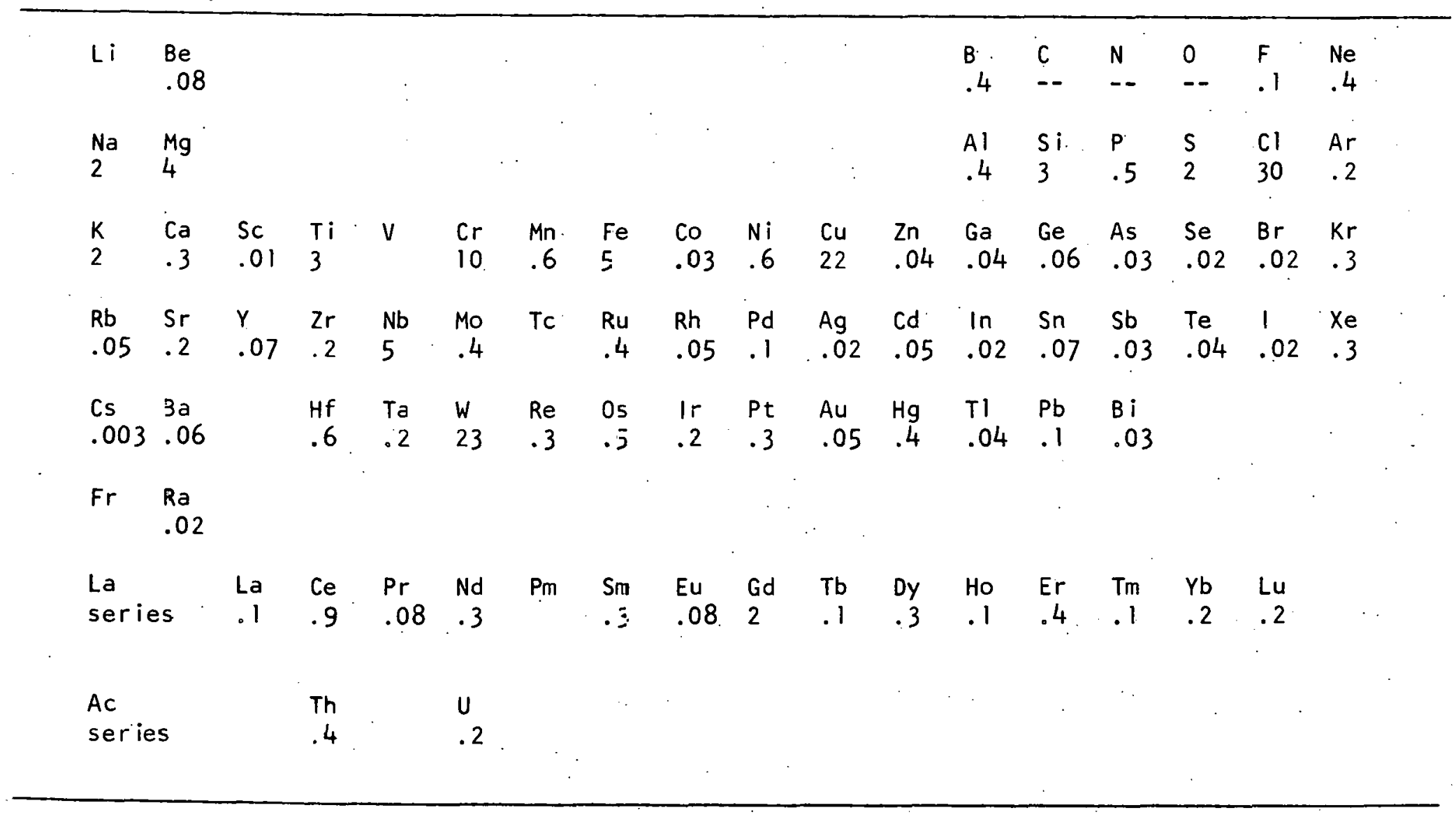

Analys is done by R. Conzemius at the Ames Laboratory, Ames, lowa.

balues are in atomic pom. 
$3 \mathrm{ppm}$. The total impurity concentration of elements (neglecting. $0, \mathrm{~N}$ and c) was $100 \mathrm{ppm}$.

Sample 3 was also SSMS analyzed for impurities. The measurement was made with the gold probe SSMS technique (47). The sample was. analyzed at fourteen locations spaced $1 \mathrm{~mm}$ apart to check for impurity gradients along the length of the sample. The major impurities in sample 3 in at. ppm are: $\mathrm{Cr}+\mathrm{VH}-100, \mathrm{~W}-12, \mathrm{Fe}-13, \mathrm{Cl}-14$ and $\mathrm{Mg}-8$. There was no evidence of an impurity gradient in sample 3 . The large concentration of chromium , plus vanadium hydride is most likely $V H$ due to surface hydrocarbön contamination and is not representative of the bulk material.'

\section{Measurement Techniques}

The sample holder is a guarded longitudinal heat flow apparatus. It is immersed in either liquid helium or liquid nitrogen in a crystat of conventional design. The temperature range of the apparatus is $4.2 \mathrm{~K}$ to $300 \mathrm{~K}$.

The electrical resistivity and thermal conductivity measurements were made with the four probe technique. The Seebeck coefficient was determined by measuring the Seebeck coefficient of the sample relative to the Seebeck coefficient of copper voltage leads. The Seebeck coefficient of the copper voltage leads was calibrated against pure lead.

Figure $I$ is a schematic of the experimental technique. The samples are cylindrical with cross-sectional area $A$. The distance between the

\footnotetext{
1 Conzemlus, R. J., Airies Laboratory, Ames, lowa, Private Commun icationn.
} 


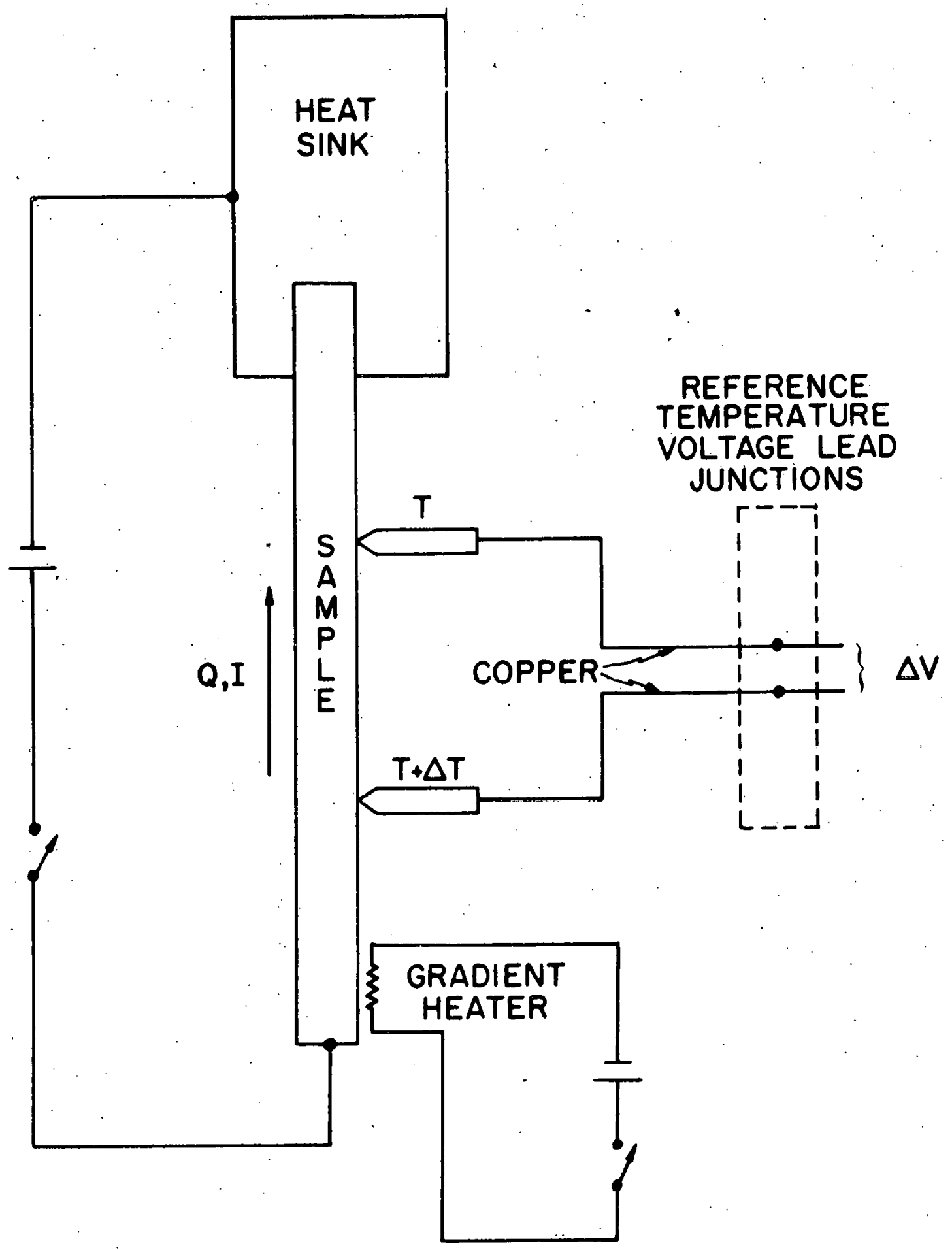

Figure 1. The four probe electrical resistivity and thermal conductivity technique. 
voltage and thermometer probes is $\ell$. The temperature of the "cold" probe is $T$ and the temperature of the "hot" probe is $T+\Delta T$ when heat $Q$ is flowing through the sample. $T_{R}$ is a reference temperature (either liquid helium or liquid nitrogen temperature) where the copper sample voltage leads make junctions with the leads of a potentiometer. $V$ is the voltage measured when electrical current 1 or heat current $Q$ passes through the sample.

Electrical resistivity measurements are made by passing electrical current 1 through the sample when the sample is isothermal; that is, $\Delta T=0$. The resistivity $\rho(T)$, is

$$
\rho(T)=\frac{A}{\ell} \frac{\Delta V}{T}
$$

Thermal conductivity measurements are made when heat $Q$ passes. through the sample and $\mathrm{I}=0$. The temperature difference $\Delta \mathrm{T}$ will vary depending upon the magnitude of the thermal conductivity $\lambda(T)$ of the sample (48). The heat current is,

$$
Q=\frac{A}{\ell} \int_{T} \cdot \lambda\left(T^{\prime}\right) \mathrm{d} l^{\prime} .
$$

This equation can be solved by expanding the thermal conductivity to first order

$$
\lambda\left(T^{\prime}\right)=B+C T^{\prime}
$$

This approximation is good for metals at low or high temperatures. It is only valid at intermediate temperatures when $\Delta T$ is small. 
Thus

$$
\begin{aligned}
Q & =\frac{A}{l}[B+C(T+\Delta T / 2)] \Delta T \\
& =\frac{A}{l} \lambda(T+1 / 2 \Delta T) \Delta T
\end{aligned}
$$

and the thermal conductivity is

$$
\lambda(T+1 / 2 \Delta T)=\frac{\ell}{A} \frac{Q}{\Delta T} \text { for small } \Delta T
$$

Seebeck coefficient measurements are also made by passing heat current through the sample when $\mathrm{I}=0$. The Seebeck voltage $\Delta V$ is

$$
\Delta V=-\int_{T_{r}}^{T} S_{C}\left(T^{\prime}\right) d T^{i}-\int_{T}^{T+\Delta T} S\left(T^{\prime}\right) d T^{\prime}-\int_{T+\Delta T}^{T} S_{C}\left(T^{\prime}\right) d T^{\prime}
$$

$$
=-\int_{T}^{T+\Delta T} S\left(T^{\prime}\right) d T^{\prime}+\int_{T}^{T+\Delta T} S_{c}\left(T^{\prime}\right) d T^{\prime}
$$

where $S\left(T^{\prime}\right)$ is the Seebeck coefficient of the sample and $S_{c}\left(T^{\prime}\right)$ is the Seebeck coefficient of copper.

The linear approximation is again made for $S\left(T^{\prime}\right)$. Hence,

$$
S(T+1 / 2 \Delta T)=\frac{1}{\Delta T} \int_{T}^{T+\Delta T} S_{c}\left(T^{\prime}\right) d T^{\prime}-\frac{\Delta V}{\Delta T}
$$


In this research the thermal conductivity and Seebeck coefficient were measured simultaneously during the same run. The electrical resistivity measurements were made in a separate run, but with the same thermometer and voltage probe spacings that were used during the thermal conductivity runs.

\section{Guarded Longitudinal Heat Flow Apparatus}

The measurement of electrical resistivity is most difficult at low temperatures where the electrical resistance of a metal is minimal. At low temperatures the residual resistances of the vanadium samples measured in this research were as low as $10^{-6} \Omega$. Consequently it was necessary to pass currents as large as I A through the samples in order to produce sample voltages of $1 \mu V$. The electrical resistivity is measured when the sample is isothermal. Thus it is important to have large electrical current leads anchored to the sample in order to prevent Joule heating in the current leads which would heat the sample. The measurement of electrical resistivity is incompatible with the measurement of thermal conductivity, since in thermal conductivity measurements, it is necessary to thermally insulate the sample gradient heater from its environment. Thermal conductivity measurements are difficult for two reasons. First, it is necessary to accurately measure the ambient temperature of the sample and the samplc temperature gradient. Second, all heat leaks from the sample must be minimized.

The thermometers were two chromel vs Au-0.03\% Fe thermocouples. One thermocouple measured the temperature of the "cold" thermometer clamp. 
The second measured the temperature gradient between the "cold" and "hot" thermometer clamps. The thermocouples were anchored to the sample as shown in Figure 2 , and were referenced to the refrigerant bath.

The heat losses from the sample heater, voltage and thermocouple leads were

$$
Q_{L}=\underset{\substack{\text { all } \\ \text { leads }}}{\sum} \frac{A}{l} \lambda \Delta T .
$$

Where $A / \ell=$ lead cross-sectional area/lead length

$$
\begin{aligned}
\lambda & =\text { lead thermal conductivity } \\
\Delta T & =\text { temperature gradient over the length of the lead. }
\end{aligned}
$$

The lead heat losses were reduced by choosing leads of low thermal conductivity and small cross-sectional area: The gradient heater leads were \#36 and \#32 manganin, the sample voltage leads were \#40 copper and the thermocouple leads were \#36 Au-0.03\% Fe and \#36 chromel.

The heat loss from radiation was

$$
Q_{R}^{\prime}=4 \pi \sigma \varepsilon a T^{3} \Delta T
$$

$$
\text { Where } \quad \begin{aligned}
\varepsilon & =\text { sample emissivity } \\
a & =\text { sample surface area } \\
\sigma & =5.6686 \times 10^{-8} \mathrm{~W} / \mathrm{m}^{2} \mathrm{~K}^{4} \\
\Delta T & =\text { temperature difference between the sample and its environ- }
\end{aligned}
$$

ment. 


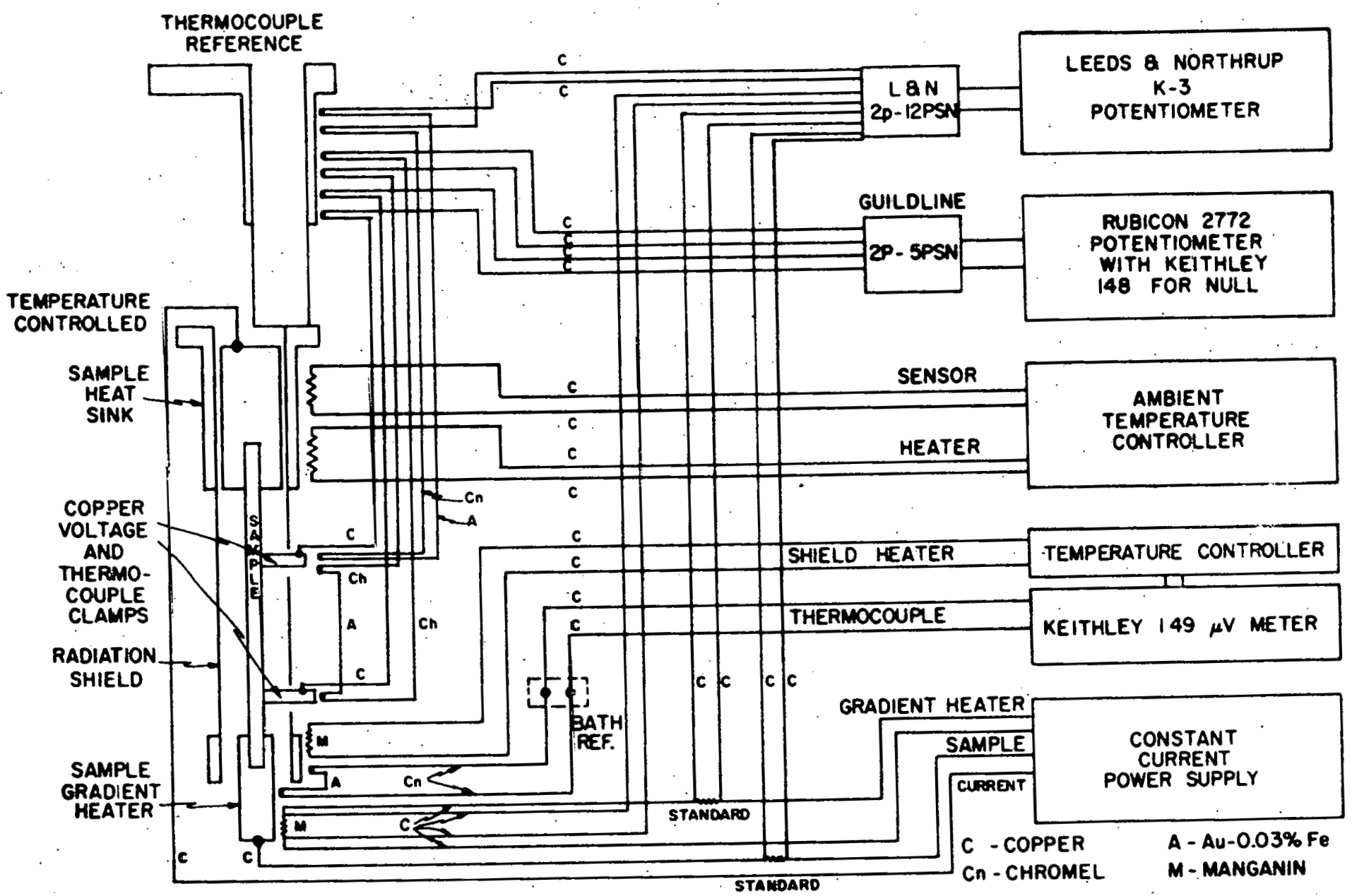

Figure 2. Sample holder wiring diagram. 
The heat losses were reduced further by surrounding the sample with a stainless-steel radiation shield as shown in Figure 3 . The shield had a gradient heater mounted on the free end which maintained a temperature gradient along the shield equal to the temperature gradient along the sample. The sample gradient heater leads were thermally anchored at junctions located on the shield heater. The thermocouple and sample voltage leads were thermally anchored on the shield on two small copper rings soldered to the shield.

When the sample and shield have thermal conductivities with the same temperature dependence the temperature gradients along the shield and sample are perfectly matched. This occurs at high temperatures where the thermal conductivity of a metal is constant. Since the sample heater, voltage and thermocouple leads were anchored on the shield in positions corresponding to their location on the sample, the temperature gradient along the leads was zero, consequently the power lost through the sample leads was zero. In addition the radiated heat loss from the sample was reduced by the radiation shield. To further reduce radiated heat loss, the entire cavity between the sample and radiation shield and between the radiation shield and first copper isothermal shield wasfilled with "Fiberfrax" insulation."

The shield temperature gradient was maintained with a temperature controller. An Au-0.03\% Fe vs chromel differential thermocouple was

\footnotetext{
'Carborundium Co., Niagara Falls, N.Y. "Fiberfrax" insulation.
} 


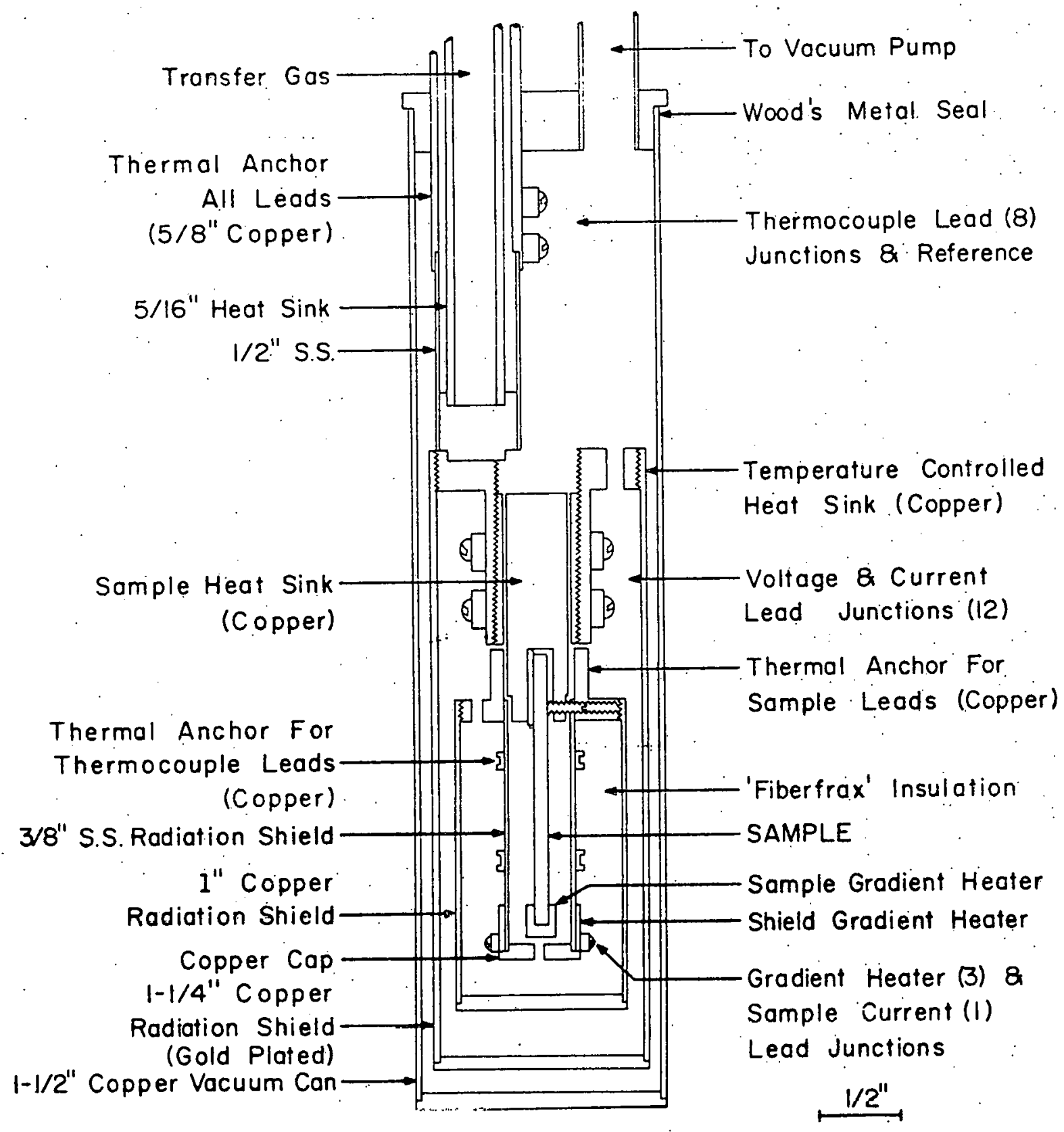

Figure 3. Thermal conductivity sample holder. 
mounted with junctions at the sample gradient heater and at the shield gradient heater. The thermocouple Seebeck voltage was monitered by a Keithley 149 micro-voltmeter as shown in Figure 2 . The $\pm 10 \mathrm{~V}$ output of the Keithley 149 wasmonitered by a temperature controller discussed in Appendix A, which held the shield heater at the same temperature as the sample gradient heater by regulating the shield heater current.

The sample temperature and voltage probes were small copper clamps tined with indium solder, which pass through a 0.1 in. slot machined along the length of the radiation shield. The thermocouple leads were thermally anchored to the copper clamps with Stycast ${ }^{\prime} 2850^{\circ} \mathrm{FT}$ epoxy with \#24LV catalyst. The copper voltage leads were soldered with cadmi um solder to the copper clamps.

The sample thermocouple and voltage leads were thermally anchored to the radiation shield with stycast epoxy as discussed above. They werethermally anchored a second time on the copper heat sink as shown in Figure 3. The copper heat sink had twelve copper posts which were the junctions for the sample heater leads with the leads that passed out of the holder. The thermocouple and sample voltage lead junctions were made at eight copper posts which were in thermal contact with the refrigerant bath.

The sample was in thermal contact with the refrigerant bath through a transfer gas chamber. The sample heat sink was temperature controlled with an a.c. resistance bridge temperature controller discussed in Appendix B. The temperature controller sensor was a $1000 \Omega, 2 \mathrm{~W}$ carbon

\footnotetext{
'Emerson and Cuming Inc., Northbrook, 111., "STYCAST"' Epoxy.
} 
resistor which was thermally anchored to the heat sink. The temperature controller held the temperature of the heat sink constant to better than $1 \mathrm{mk}$ at low temperatures.

The sample holder had three radiation. shields. One was the stainlesssteel shield discussed above. The other two were copper shields which were threaded to fit on the sample heat sink as shown in Figure 3 . The entire sample holder assembly was contained in a 1.5 in. diameter copper vacuum can which was soldered with "Woods" metal to a flange in thermal contact with the refrigerent bath. The vacuum system waspumped with a standard oil diffusion pump to pressures lower than $5 \times 10^{-5}$ Torr.

Figure 2 is a block diagram of the sample holder wiring and electronics. The sample current for electrical resistivity measurements and the gradient heater current for thermal conductivity and Seebeck coefficient measurements were supplied by a constant current power supply (49). The power. supply had two independent outputs which supplied currents of $0.1 \mathrm{~A}$ to $2 \mathrm{~A}$ and $0.1 \mathrm{~mA}$ to $63 \mathrm{~mA}$. The current regulation was to $0.02 \%$.

A Leeds and Northrup K-3 potentiometer measured the sample absolute thermocouple EMF, the sample gradient heater EMF, the voltage across a precision resistor in series with the sample gradient heater during thermal conductivity runs and the voltage across a precision resistor in series with the sample during electrical resistivity runs. The two standard resistors were needed to measure the gradient heater current during thermal conductivity runs and the sample current during electrical resistivity runs. 
Thermometry

In order to reduce heat losses from the sample the temperature of the "cold" thermometer clamp and the sample temperature gradient were measured with thermocouples rather than with resistance thermometers. The resistance thermometers commerically available, either germanium or platinum are physically large with respect to the sample. Consequently at high temperatures the thermometers radiate considerable energy which is difficult to shield for large thermometers. In addition the thermocouples span a wide range of temperature. In order to take data from $4 \mathrm{~K}$ to $300 \mathrm{~K}$ two sets of resistance thermometers would be needed while one set of thermocouples is sufficient.

- The Au-0.03\% Fe vs chromel absolute thermocouple was referenced to the refrigerent bath. The bath temperaturewas measured by measuring the bath vapor pressure with a mercury column manometer. The resolution of the manometer was $0.5 \mathrm{~mm}$ and this permitted the bath temperature to be measured to $2 \mathrm{mK}$. The helium bath temperature wascalculated from the 1958 helium temperature scale, the nitrogen bath temperature wascalculated from the data of Corrunccini (50).

The thermocouples were calibrated after they were mounted in the sample holder. From $4.2 \mathrm{~K}$ to $80 \mathrm{~K}$ the calibration was made with two germanium resistance thermometers purchased from cryo-Cal Inc.!. These thermometers were calibrated by Marvin Anderson at the Ames Laboratory.

'Cryo-Cal Inc., Riviera Beach, Florida. 
The absolute thermocouple and the differential thermocouple were calibrated independently from $4.2 \mathrm{~K}$ to $80 \mathrm{~K}$. Two small copper thermometer holders were thermally anchored to the two thermocouple thermometer clamps. The "cold" germanium thermometer was in thermal contact with the sample-holder heat-sink and the heat sink temperature was held constant with the ambient temperature controller. The "hot" germanium thermometer was in thermal contact with the shield gradient heater and its temperature could be raised above the "cold" thermometer with the shield heater. The Seebeck $\operatorname{EMF}\left(V_{T}\right)$ of the absolute thermocouple as a function of temperature $(T)$ and bath temperature $\left(T_{B}\right)$ was measured. These data were fit to polynomials with the least squares technique.

The Seebeck EMF $\left(V_{\Delta T}\right)$ of the differential thermocouple was measured as a function of "cold" temperalure $(T)$ and temperature gradient $(\Delta T)$. The relative Seebeck coefficient $\left(V_{\Delta T} / \Delta T\right)$ was then fitted to polynomals. Above $80 \mathrm{~K}$ only the absolute thermocouple was calibrated. The calibration was made with a platinum resistance thermometer from Leeds and Northrup Corp. The thermometer was calibrated by NBS in 1967. The calibration of the absolute thermocouple above $80 \mathrm{~K}$ was made in the same manner as the calibration made below $80 \mathrm{~K}$. The differential thermocouple calibration above $80 \mathrm{~K}$ was made by calculating the relative Seebeck coefficient of Au-0.03\% Fe vs chromel from the fit of the absolute thermocouple.

$$
S_{R}(T)=-\frac{d V}{d T}
$$


The fitting of the thermocouples and an analysis of the temperature errors is discussed in Appendix $C$.

\section{Calibration of Seebeck Coefficient}

A cylindrical sample of pure lead was prepared in a graphite crucible In an R-F furnice. This sample was the standard for the Seebeck coefficient calibration of the copper voltage leads.

The lead sample was mounted in the sample holder and the relative Seebeck coefficient of copper vs lead was measured.

$$
\begin{aligned}
S_{R}(T) & =-\frac{\Delta V}{\Delta T} \\
& =S_{P b}(T)-S_{C}(T)
\end{aligned}
$$

where $S_{P b}(T)$ is the Seebeck coefficient of lead and $S_{C}(T)$ is the Seebeck coefficient of copper. The absolute Seebeck coefficient of the copper leads was calculated from equation (50) using the absolute Seebeck coefficient data of lead as measured by Christian et al. (51). The copper Seebeck coefficient data were fitted to polynumials by the leastsquares method.

\section{Measurement of Form Factor}

The electrical resistivity of each sample was measured in a special sample holder by the four probe technique at room temperature and at $273 \mathrm{~K}$. The voltage probe separation and sample cross-sectional area were measured with a traveling microscope. 
The form factor (voltage probe separation/cross-sectional area) of the sample mounted in the thermal conductivity holder was calculated by comparing the electrical resistance of the sample after it had been mounted in the thermal conductivity holder with the previously measured electrical resistivity.

$$
\frac{1}{F}=\frac{\rho_{296 K} K}{R_{296 K}}=\frac{\rho_{273 K} K}{R_{273 K}}
$$

The resistance of the sample in the thermal conductivity holder at $273 \mathrm{~K}$ was measured by immersing the sample holder in an ice bath.

\section{Techniques for Data Taking}

The sample was mounted in the sample holder. A \#20 Cu wire was soldered with indium." solder to the sample gradient heater and to a iunction on the shield gradient heater. A complete electrical circuit was thus provided for the sample current during electricial resistivity runs. The sample form factor was measured in the manner just described.

Electrical resistivity data were taken as follows. The ambient temperature of the sample was regulated with the a.c. temperature controller. When the sample reached thermal equilibrium the sample current was turned on. It required from several seconds at $5 \mathrm{~K}$ to several hours at an ambient temperature of $250 \mathrm{~K}$ for the sample to reach thermal equilibrium. The absolute thermocouple voltage $V_{T}$ and bath pressure $P_{B}$ were recorded. The sample voltage $V_{S}$ and the voltage across the standard resistor $V_{S T D}$ were recorded for both forward and reverse 
sample current directions. The resistivity of the sample was

$$
\rho=\frac{1}{F} \frac{V_{S}(F)+V_{S}(R)}{V_{S T D}(F)+V_{S T D}(R)}{ }^{R_{S T D}}
$$

where $R_{\text {STD }}=$ resistance of the standard resistor in series with the sample.

Thermal conductivity and Seebeck coefficient measurements were. made after the electrical resistivity run was completed. The \#20 Cu wire was removed from the sample in order to thermally insulate the sample gradient heater from the radiation shield. Thermal conductivity and Seebeck coefficient measurements were made as follows. The ambient temperature of the sample was regulated with the a.c. temperature controller and the sample was allowed to come to thermal equilibrium. The differential thermocouple offset voltage $V^{\prime}{ }_{\Delta T}$ ' the sample Seebeck offset voltage $V_{S}^{\prime}$ and the bath pressure $P_{B}$ were recorded. The offset voltage of the differential thermocouple for the shield-gradient heater was bucked by adjusting the zero suppress on the Keithley 149 micro-voltmeter. The shield-gradient heater temperature controller and the sample gradient heater were turned on. When the sample reached the steady state heat flow $\left(\frac{d T}{d t}=0\right)$ condition the thermal conductivity and Seebeck coefficient were measured. The absolute thermocouple voltage $V_{T}$, the differential thermorouple voltage $V_{\Delta T}$, the sample Seebeck voltage $V_{S}$, the gradient heater voltage $V_{H}$ and the gradient heater current voltage $v_{1}$ across standard resistor $R_{S T D}$ were recorded for forward and reverse directions 
of the gradient-heater current. The temperature of the "cold" thermocouple (T) was calculated from $V_{T}$ and $P_{B}$ from the thermocouple fit. The sample temperature difference $(\Delta T)$ was calculated from $T$ and $V T^{-} V_{T}{ }^{\prime}$ from the differential thermocouple fit. The thermal conductivity of the sample was

$$
\lambda(T+1 / 2 \Delta T)=F \frac{\left(V_{H}(F)+V_{H}(R)\right)\left(V_{1}(F)+V_{1}(R)\right)}{4 R_{S T D}} .
$$

The sample Seebeck coefficient was

$$
S(T+1 / 2 \Delta T)=\frac{1}{\Delta T} \int_{T}^{T+\Delta T} S_{C}\left(T^{\prime}\right) d T^{\prime}-\frac{V_{S}-V_{S}{ }^{\prime}}{\Delta T}
$$

At intermediate temperatures the thermal conductivity is a rapidly varying function of temperature and the linear solution to equation 41 is not valid. In this temperature region several measurements of sample temperature difference $\Delta T$, sample "cold" thermometer temperature $T$ and gradient heater power $Q$ were made for one ambient temperature setting. The temperature gradients were varied from $0.1 \mathrm{~K}$ to $\mathrm{l} \mathrm{K}$.

Equation 41 was solved by expanding the thermal conductivity as

$$
\lambda(T)=K_{1}+K_{2} T+K_{3} T^{2}+\cdots \cdot+K_{n} T^{n-1}
$$

where $n=$ number of measurements of $\Delta T$ and $T$ vs $Q$ at constant ambient temperature. The solution to the heat flow equation 41 in terms of the expansion 55 is 


$$
Q=\frac{1}{F} \sum_{i=1}^{n} \frac{K_{i}}{i}\left[(T+\Delta T)^{i}-T^{i}\right] .
$$

The $n$ coefficients $K_{i}$ were calculated from the $n$ data points $T$ and $\Delta T$ vs $Q$ with least squares. Thus at the temperature

$$
T^{\prime}=\frac{1}{n} \sum_{i=1}^{n}\left[T_{i}+1 / 2 \Delta T_{i}\right]
$$

the thermal conductivity is

$$
\lambda\left(T^{\prime}\right)=\sum_{i=1}^{n} K_{i}\left(T^{\prime}\right)^{i-1} .
$$

The above general expansion equals the linear expansions when $n=1$.

\section{Experimental Errors}

Our major experimental uncertainity in the determination of electrical resistivity and thermal conductivity was the measurement of the sample form factor. The sample diameter and voltage probe separation were measured with a traveling microscope with a precision of $0.01 \mathrm{~mm}$. The sample voltage probe separation was typically $1 \mathrm{~cm}$ and the sample diameter was typically $0.25 \mathrm{~cm}$. Thus the imprecision in measuring the sample form factor was $0.8 \%$.

The vanadium samples had the bamboo structure typical of electrotransported metals. Consequently the samples had cross-sectional areas which were non-uniform over the length of the sample. The worst 
sample was sample 2 which had a cross-sectional area which varied as much as $3 \%$ between the thermometer probes.

The least squares fitting and error analysis of the thermocouple cal ibration is discussed in Appendix $C$. The temperature measurement uncertainty was large for temperatures between $70 \mathrm{~K}$ to $80 \mathrm{~K}$ and for temperatures greater than $280 \mathrm{~K}$. Over the rest of the temperature range the temperature uncertainty was less than $60 \mathrm{mK}$.

The sample temperature difference measurement had an imprecision which varied with temperature as discussed in Appendix $C$. The error in temperature difference measurement was largest at low temperatures where small temperature differences were needed. The sample temperature differences in this research varied from $0.1 \mathrm{~K}$ to $0.5 \mathrm{~K}$ for temperatures between $5 \mathrm{~K}$ to $30 \mathrm{~K}, 0.25 \mathrm{~K}$ to $\mathrm{I} \mathrm{K}$ for temperatures between $30 \mathrm{~K}$. to $100 \mathrm{~K}$ and $0.5 \mathrm{~K}$ to $3 \mathrm{~K}$ for temperatures between $100 \mathrm{~K}$ to $300 \mathrm{~K}$. Consequently at low temperatures the error in the temperature difference measurement was as large as $0.5 \%$.

As discussed in Appendlx $C$, the callbration of the differential thermocouple from $80 \mathrm{~K}$ to $90 \mathrm{~K}$ was in error due to the difficulty in fitting the differential thermocouple from $80 \mathrm{~K}$ to $90 \mathrm{~K}$. Over the temperature range $80 \mathrm{~K}$ to $90 \mathrm{~K}$ the error in the temperature difference measurement was large and no data were taken in this temperature range. 
The imprecision in thermal conductivity measurement varied with temperature due to the imprecision in temperature measurement, temperature difference measurement and heat current measurement. The error in the form factor measurement was constant over the entire temperature range. Bolef et al. (13) have shown that the thermal expansion of vanadium was less than $0.15 \%$ from $273 \mathrm{~K}$ to $5 \mathrm{~K}$,

The uncertainty in the heat current was due to heat losses from the sample through the sample electrical leads and from radiation. The heat losses from the sample were reduced by the radiation shield surrounding the sample. When the shield and sample temperature gradients were perfectly matched the sample heat losses due to the leads werezero and the sample heat current was the Joule heating in the sample gradient heater. The gradient heater power measurement imprecision wasless than $0.05 \%$

The imprecision in the thermal conductivity measurements due to the form factor error and temperature gradient error was $1 \%$ at low temperatures and $0.8 \%$ at high temperatures. The error in the sample temperature measurement ( $1 \%$ at $5 \mathrm{~K}, 0.3 \%$ at $10 \mathrm{~K}$ ) introduced an additional imprecision in the thermal conductivity measurement at low temperatures. The magnitude of the uncertainty depended upon the slope of the sample's thermal conductivity.

The systematic errors in this research weredue to the non-uniform sample cross-sectional areas and the sample heat losses. The systematic errors were analyzed by measuring the thermal conductivity of a sample 
of electrolytic iron, SRM 734. The systematic errors wereless than $3 \%$

The imprecision in electrical resistivity measurement wasdue to the uncertainty in the sample form factor $(0.8 \%)$ and the uncertainty in measuring the sample resistance. At low temperatures where the resistivity wasminimal, the electrical resistivity errors were the greatest. The sample voltage was measured with a precision of $0.005 \mu \mathrm{V}$ and the sample current was measured with a precision of $0.5 \mathrm{~mA}$. At high temperatures where the sample resistance was greater than $5 \times 10^{-5} \Omega$ the electrical resistivity data have an imprecision determined primarily by the imprecision in measuring the sample form factor $(0.8 \%)$. At low temperatures the sample resistances were as low as $5 \times 10^{-7} \Omega$. Consequently the imprecision in electrical resistivity measurements at low temperatures was $1.3 \%$ for the highest purity vanadium sample, sample 4.

The Seebeck coefficient imprecision wasdue to the uncertainty in measuring the sample temperature gradient and the uncertainty in meașuring the sample Seebeck EMF. Below $10 \mathrm{~K}$ vanadium had a Seebeck coefficient of less than $0.1 \mu \mathrm{V} / \mathrm{K}$. The Seebeck voltage, $V_{S}$ was measured with a precision of $0.005 \mu \mathrm{V}$. Since the Seebeck coefficient was measured simultaneously with the thermal conductivity measurements, the temperature gradients were less than $0.5 \mathrm{~K}$ for $\mathrm{T}<10 \mathrm{~K}$. Consequently the error in the Seebeck coefficient below $10 \mathrm{k}$ was greater than $10 \%$. Above $10 \mathrm{~K}$ the Seebeck coefficient rose to approximately $3 \mu \mathrm{V} / \mathrm{K}$ at $50 \mathrm{~K}$. Hence the uncertainty in the Seebeck coefficient for temperatures above $20 \mathrm{~K}$ 
wasless than $3 \%$. At high temperatures the Seebeck coefficient was again small (less than $l \mu V / K$ ). However at high temperatures the sample temperature gradients were large (greater than $2 \mathrm{~K}$ ). Thus the imprecision in the Seebeck coefficient at high temperatures was also about $3 \%$.

\section{Electrolytic Iron}

A sample of electrolytic iron, SRM 734 was obtained from the National Bureau of Standards (NBS). This material is a thermal conductivity, electrical resistivity and Seebeck coefficient standard. The transport properties of SRM 734 were measured to test the expeimental uncertainty of the apparatus.

The sample received from NBS was cylindrical with a length of $12 \mathrm{in.}$ and a diameter of $0.21 \mathrm{in.} \mathrm{A} \mathrm{smaller} \mathrm{sample} \mathrm{was} \mathrm{prepared} \mathrm{from} \mathrm{the} \mathrm{bulk}$ by cutting a $3.5 \mathrm{~cm}$ piece from the rod and grinding the sample to a diameter of $0.25 \mathrm{~cm}$. The sample was electropolished and annealed. The annealing was conducted in a programable furnice which had a pressure of less than $5 \times 10^{-5}$ torr. The vacuum system was a standard oil. diffusion pump. The sample was heated to $1000^{\circ} \mathrm{C}$ at a heating rate of $100^{\circ} \mathrm{C} / \mathrm{h}$. The sample was held at $1000^{\circ} \mathrm{C}$ for two hours and then cooled to $775^{\circ} \mathrm{C}$ in one hour. The sample was held at $775^{\circ} \mathrm{C}$ for four hours and then cooled to room temperature at a cooling rate of $200^{\circ} \mathrm{C} / \mathrm{h}$. After the anneal the sample was again electropolished. The characteristics of the annealed sample are listed in Table 3. 
Table 3. Characteristics of sample SRM 734

\begin{tabular}{lcccc}
\hline Length & Diameter & $\begin{array}{c}\text { Resistivity } \\
(\mathrm{T}=273 \mathrm{~K})\end{array}$ & $\begin{array}{c}\text { Residual } \\
\text { Resistivity }\end{array}$ & $\begin{array}{c}\text { Resistance Ratio } \\
\rho_{273} / \rho_{4.2}\end{array}$ \\
\hline $31 / 2 \mathrm{~cm}$ & $0.2492 \mathrm{~cm}$ & $9.022 \mu \Omega \mathrm{cm}$ & $0.3709 \mu \Omega \mathrm{cm}$ & 24.3 \\
\hline
\end{tabular}

Hust and Sparks $(52,53)$ reported that samples annealed at NBS for two hours at $1000^{\circ} \mathrm{C}$ had resistance ratios of $23.33 \pm 0.24$. The resistance ratio of our sample was $4 \%$ higher than the resistance ratio reported by NBS. NBS reported, however, that samples annealed for $21 / 2$ days at $1000^{\circ} \mathrm{C}$ were $2.6 \%$ higher than the resistance ratio of the sample measured in this research. No explanation of the variation of resistance ratio with different anneal temperatures has been made. However, Hust 1 has found that samples of SRM 734 annealed at $1000^{\circ} \mathrm{C}$ for two hours, and subsequently annealed at $800^{\circ} \mathrm{C}$ for, four hours have resistance ratios which are the same.

The thermal conductivity of sample SRM 734 is shown in Figure 4 along with the results of NBS. Figures 5 and 6 are plots of the electrical resistivity and Seebeck coefficient compared to NBS. Figure 7 is a plot of the per cent deviation of our measurements from the results of NBS of all three transport properties.

\footnotetext{
IHust, J. G., NBS, Boulder, Colorado, Private Communication.
} 


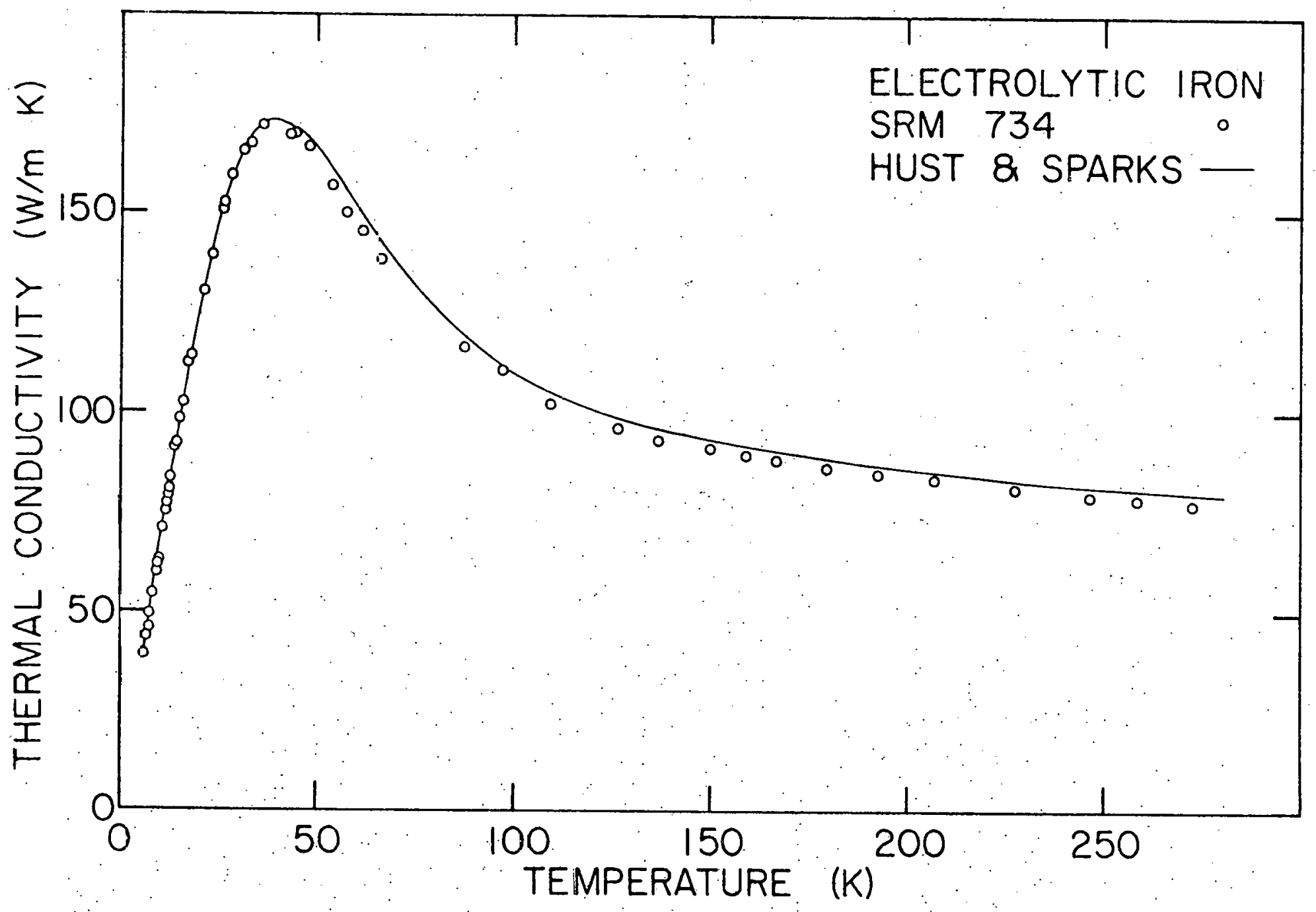

Figure 4. The thermal conductivity of electrolytic iron as a function of temperature. 


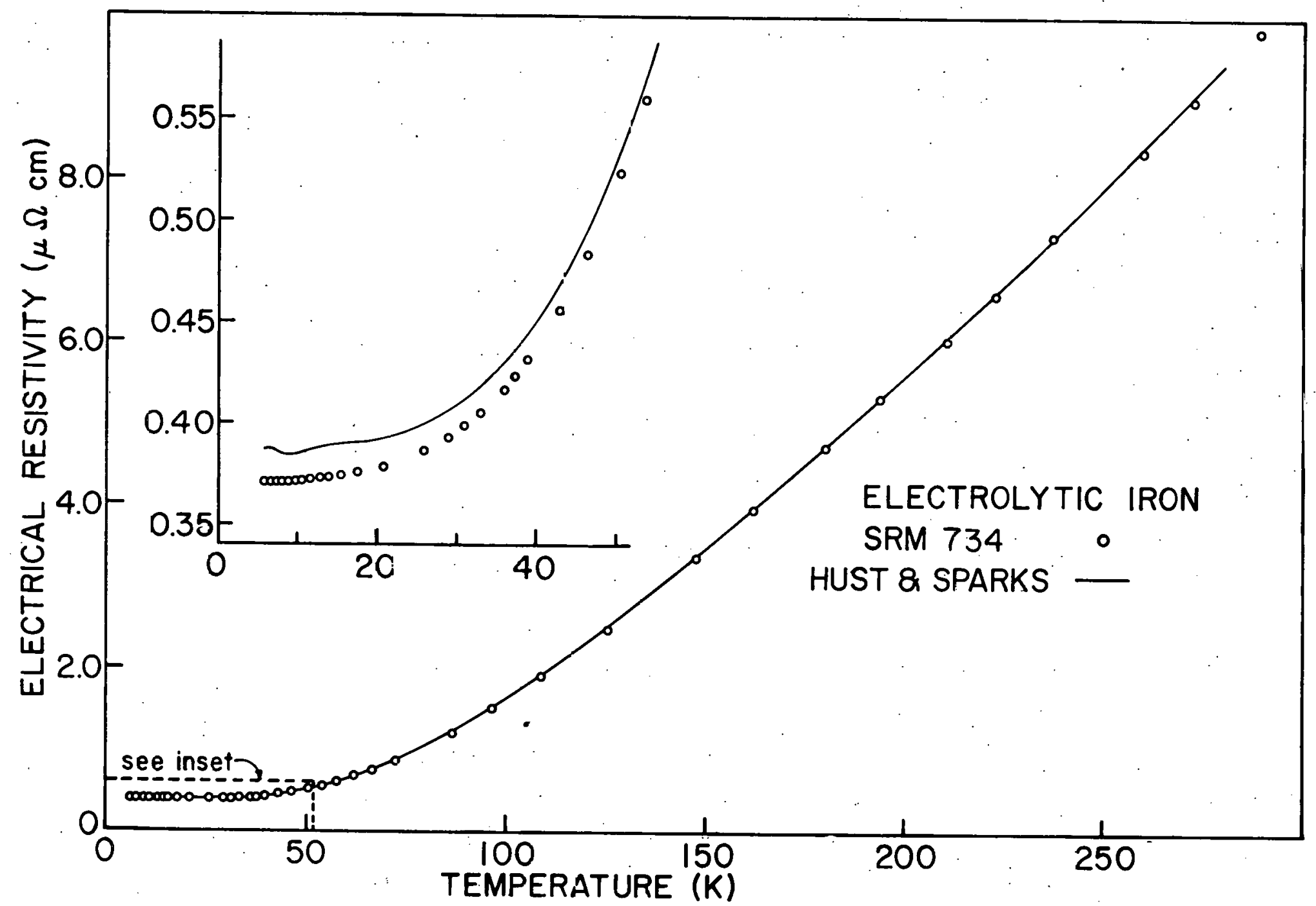

s

Figure 5. The electrical resistivity of electrolytic iron as a function of temperature. 


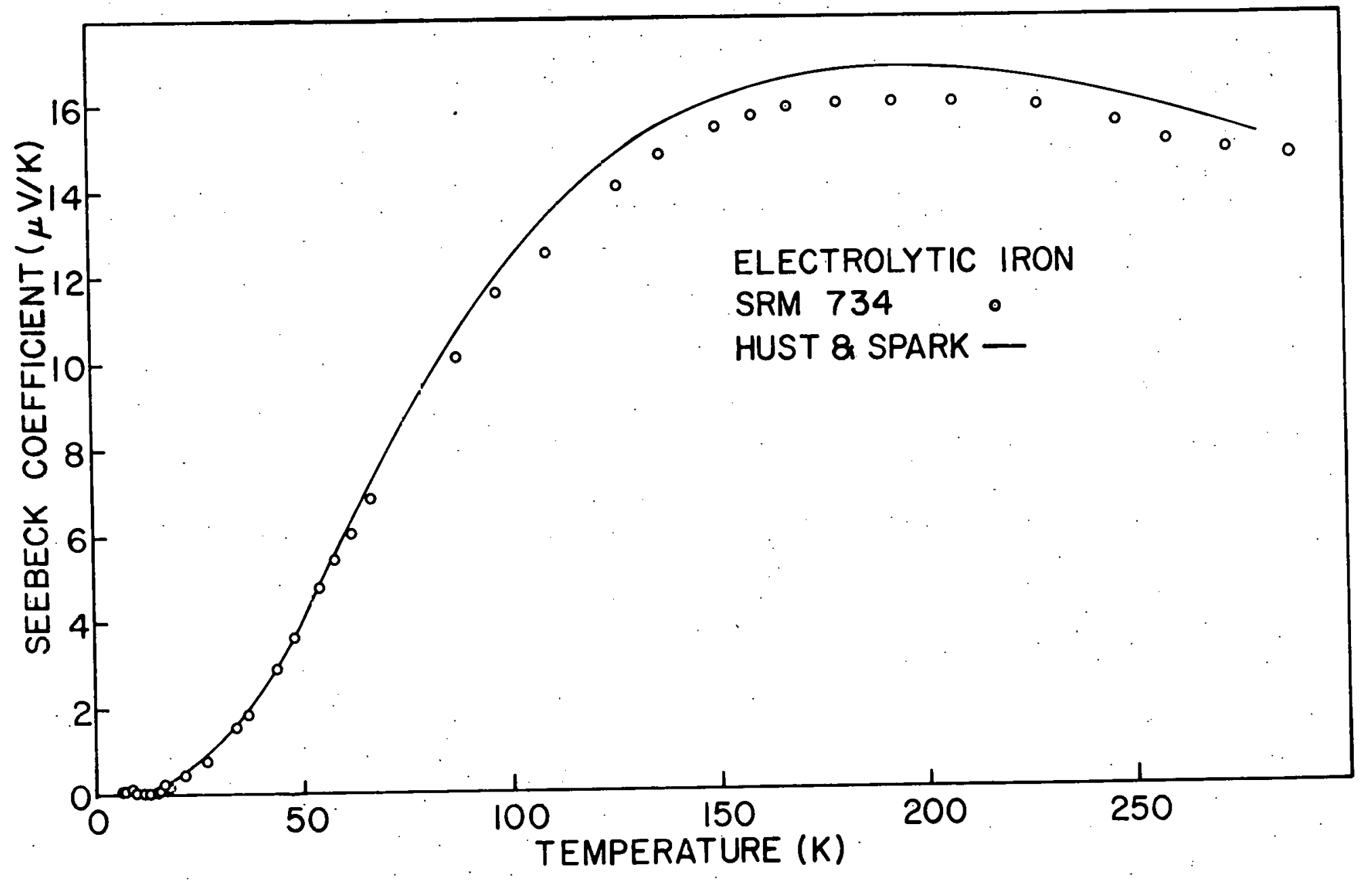

Figure 6. The Seebeck coefficient of electrolytic iron as a function of temperature. 


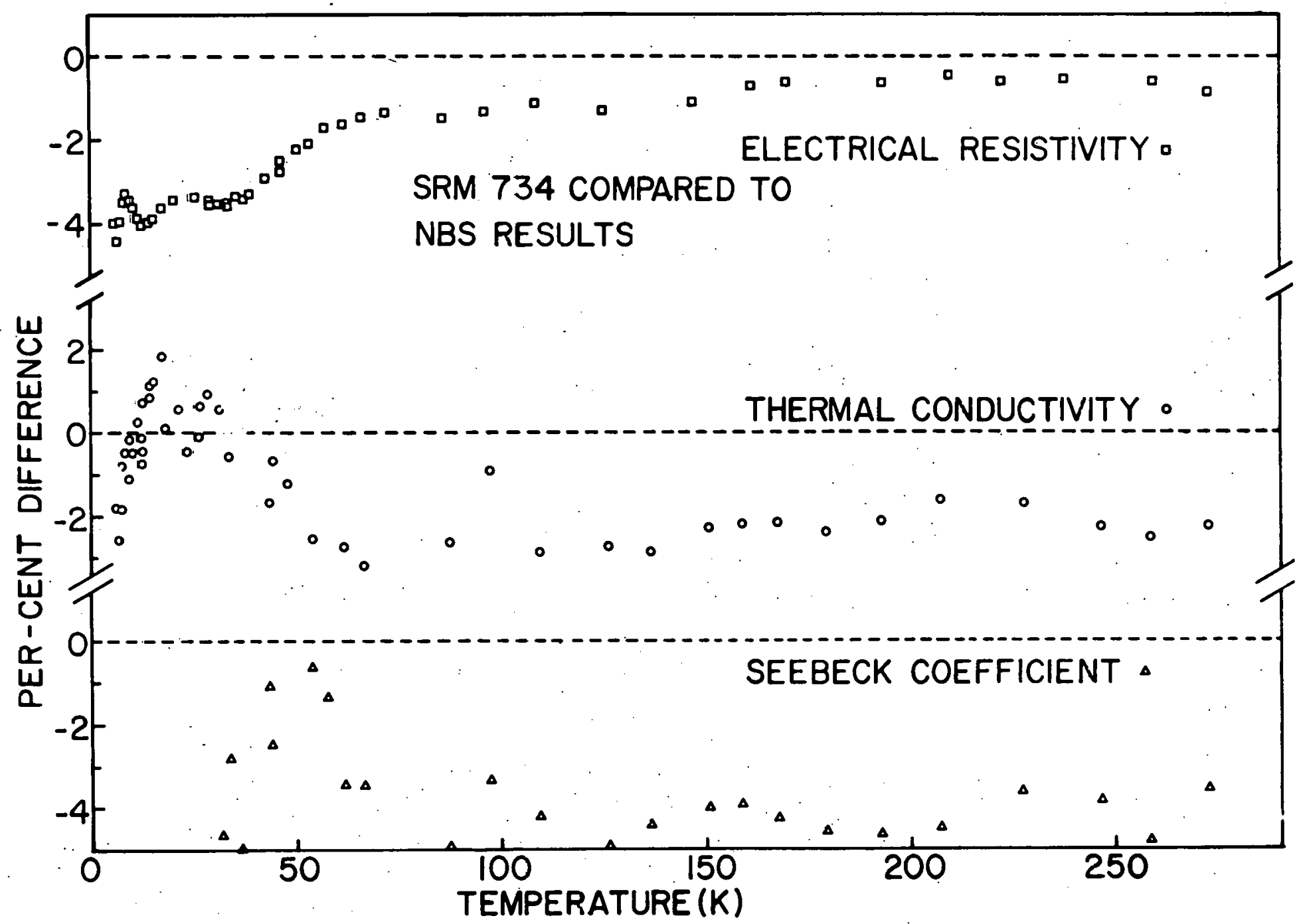

Figure 7. Comparison of the the-mal conductivity, electrical resistivity and Seebeck coefficient of electrolytic iron to the results of NBS. 
Above $160 \mathrm{~K}$ the electrical resistivity of SRM 734 was in good agreement with the result of NBS. At low temperatures however our measurements were $4 \%$ less than the NBS results. In addition the structure present in the low temperature electrical resistivity reported by NBS was not present in our sample. The imprecision in the electrical resistivity data was $0.8 \%$ over the entire temperature range. The difference in our data with respect to NBS was due to variations in SRM 734 samples. We know of no temperature dependent systematic error which could cause our electrical resistivity data to deviate $4 \%$ at low temperatures.

The thermal conductivity measurement of SRM 734 was in good agreement with NBS for temperatures less than $40 \mathrm{~K}$. The agreement between the thermal conductivity data at low temperatures was puzzling since the electrical resistivity data indicates the sample was different from the samples measured at NBS. Above $40 \mathrm{~K}$ however our thermal conductivity data was $3 \%$ less than the NBS results. This disagreement was larger than the imprecision of our apparatus in this temperature range. Consequently a systematic error of approximately $3 \%$ was present in the thermal conductivity measurements for temperatures above $40 \mathrm{~K}$.

The Seebeck coefficient, shown in Figure 6 , was $4 \%$ less than the results of NBS for temperatures greater than $40 \mathrm{~K}$. Below $30 \mathrm{~K}$ the errors in Seebeck coefficient exceeded $10 \%$. Since the Seebeck coefficient measurement and thermal conductivity measurement both depend upon the measurement of temperature gradient, the temperature dependent systematic error in this research above $40 \mathrm{~K}$ was most likely an uncertainty in measuring the sample temperature difference. The systematic uncertainty 
in the temperature difference measurement was due to a systematic uncertainty in measuring the Seebeck coefficient of chromel.vs Au-0.03\% Fe above $40 \mathrm{~K}$. 
CHAPTER IV: RESULTS

Electrical Resistivity

Figure 8 is a plot of the electrical resistivity of vanadium as a function of temperature from $5.4 \mathrm{~K}$ to $290 \mathrm{~K}$. The three highest purity samples had electrical resistivities which were equal above $130 \mathrm{~K}$. We observed no resistivity anomaly at intermediate temperatures in agreement with Westlake (25) who reported that hydrogen increased the electrical resistivity of vanadium above $190 \mathrm{~K}$. The hydrogen content in our samples was less than 1 wt. ppm. The highest purity vanadium sample, sample, 4 had a residual resistivity of $1.3 \times 10^{-8} \Omega \mathrm{cm}$. We observed with our electrical resistivity measurements that the superconducting transition temperature of vanadium was $5.4 \mathrm{~K}$ in agreement with the results of Radebaugh and Keesom (12). The lowest purity sample, sample 1, had an electrical resistivity which was larger than the other four samples over the entire temperature range.

Matthiessen's rule was applied to the electrical resistivity in order Lu separace the residual and ideal (intrinsic) electrical resistivities.

$$
\rho_{i}(T)=\rho_{i}(T)-\rho_{0}
$$

where $\rho_{0}$ is the residual electrical resistivity, $\rho(T)$ is the total resistivity and $\rho_{i}(T)$ is the ideal resistivity. The residual resistivity for each sample was the resistivity measured at the lowest temperature. The temperature dependence of the ideal electrical resistivity was calculated by plotting $\log \left(p_{i}\right)$ vs $\log (T)$ as shown in Figure 9. As seen 


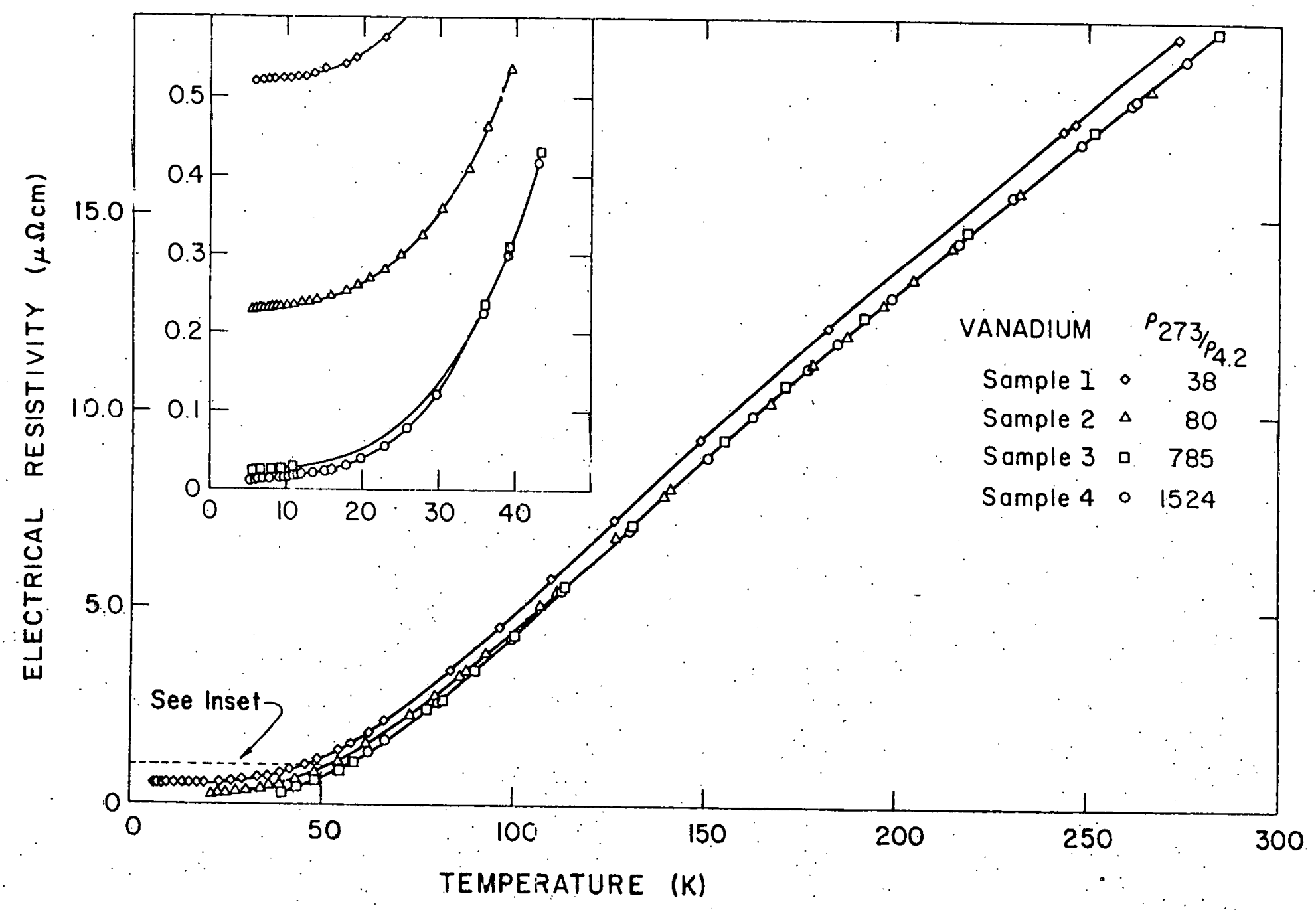

Figure 8. The electrical resistivity of vanadium as a function of temperature for four samples of high purity vanadium. The lowest purity sample, sample 1 , had an electrical resistivity which was affected by impurities over the entire temperature range. 


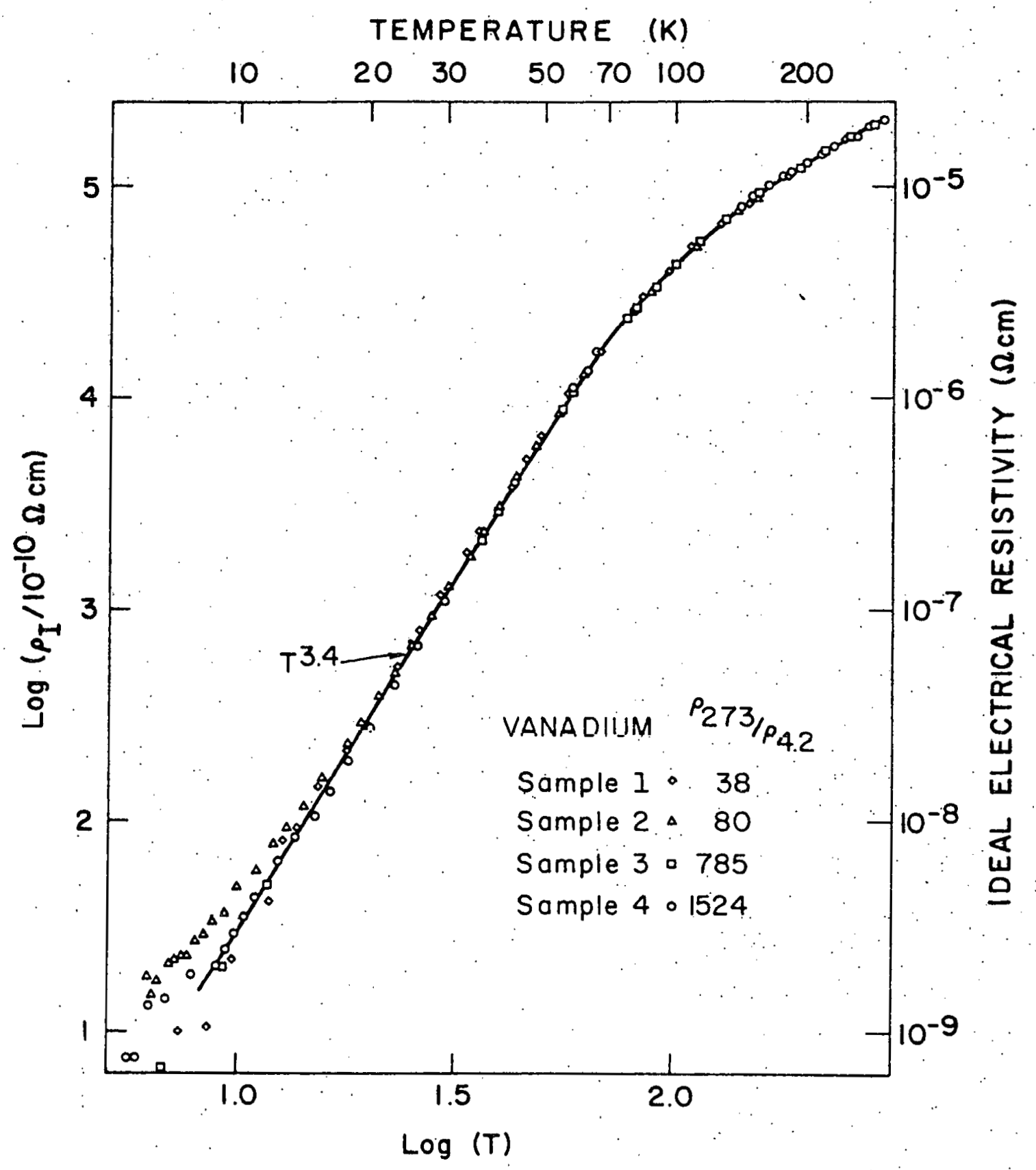

Figure 9. The log of the ideal electrical resistivity plotted as a - function of the log of the temperature. Vanadium had an ideal electrical. resistivity which varied as $T^{3.4}$ from $10 \mathrm{~K}$ to $50 \mathrm{~K}$. 
in Figure 9, the ideal electrical resistivity of vanadium had a $T^{3,4}$ temperature dependence between $10 \mathrm{~K}$ and $70 \mathrm{~K}$. Our results were in close agreement with White and Woods (19) who found that the ideal electrical resistivity of vanadium varied as $\mathrm{T}^{3.35}$ at low temperatures. The lowest purity sample, sample 1 , had an ideal electrical resistivity which was in agreement with the other four samples over the entire temperature range. Thus the excess electrical resistivity of this sample, as seen in Figure 8 , was due to point impurity scattering, which affected the total electrical resistivity of sample 1 over the entire temperature range.

\section{Thermal Conductivity}

The thermal conductivity of vanadium is plotted as a function of temperature in Figure 10. Also plotted in Figure 10 are the results of White and Woods (19). As seen in Figure 10, the thermal conductivity of vanadium approached a constant at room temperature and had a large maximum at low temperatures. The highest purity sample, sample 4, had a thermal conductivity maximum of $920 \mathrm{~W} / \mathrm{m} \mathrm{K}$ at $9 \mathrm{~K}$. The three highest purity samples all have equal thermal conductivities above $60 \mathrm{~K}$ and all three samples had thermal conductivities of $35 \mathrm{~W} / \mathrm{m} \mathrm{K}$ at room temperature. The thermal conductivity maximum of our highest purity sample was over 500 times greater than the thermal conductivity of a much less pure sample reported by White and Woods. As seen in Figure 10, the thermal conductivity of our lowest purity sample, sample 1 , and the thermal conductivity results of White and Woods were less than the thermal conductivities of the other three higher purity samples over the entire temperature range. The 


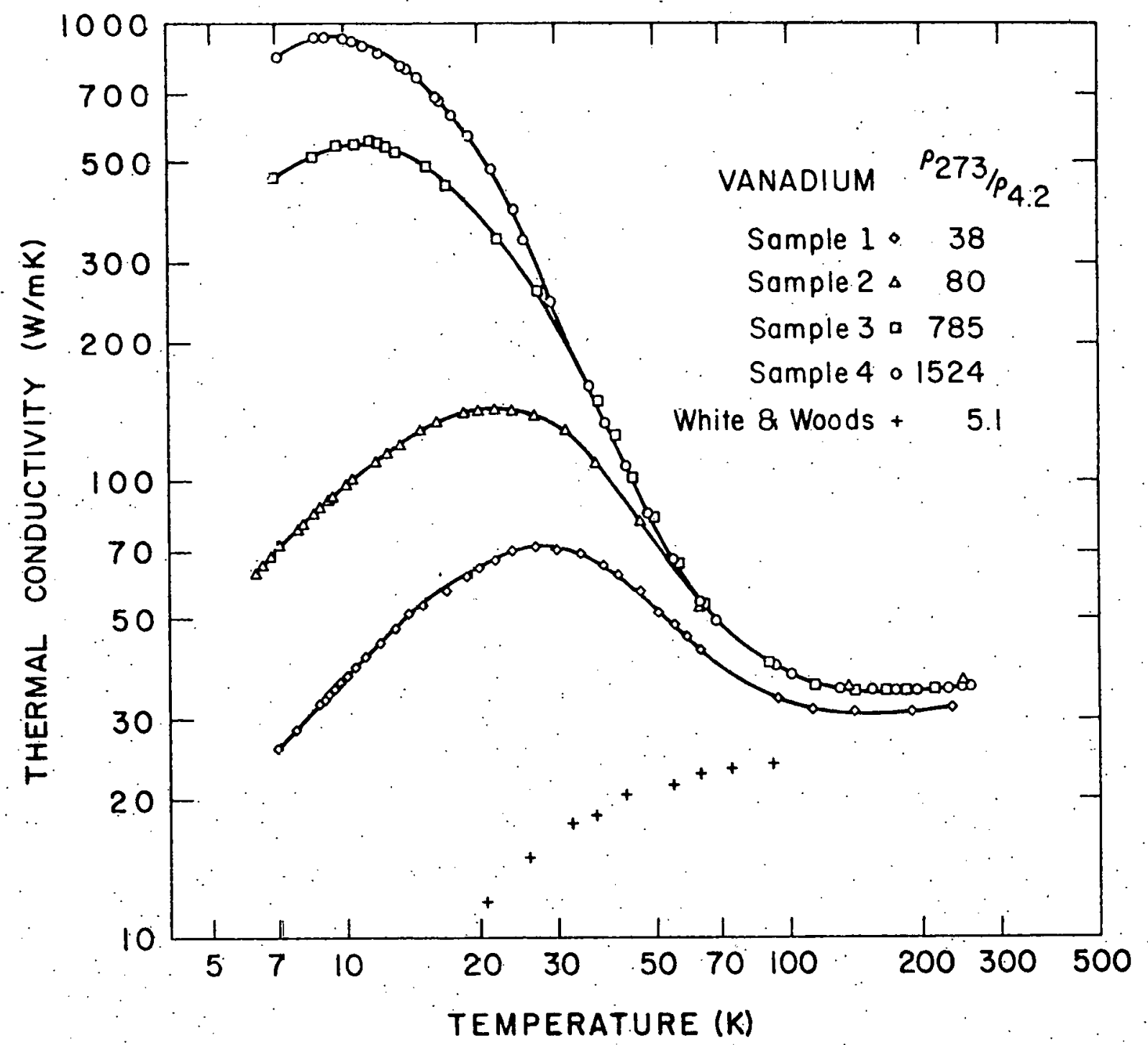

Figure 10. The thermal conductivity of vanadium as a function of temperature. The lowest purity sample, sample 1 , had a thermal. conductivity which was limited by impurity scattering over the entire temperature range. 
The difference in thermal conductivity of the lowest purity sample was due to point impurity scattering.

The thermal analogue of Matthiessen's rule was applied to the thermal resistivity.

$$
W(T)=A / T+W_{i}(T)
$$

where $W_{i}(T)$ is the intrinsic thermal resistivity and $A / T$ is the thermal resistivity due to point impurity scattering. The constant $A$ was calculated from the slope of the low temperature thermal conductivity and the intrinsic thermal resistivity was calculated from equation 60 . The temperature dependence of the intrinsic thermal resistivity was evaluated by plotting $\log w_{i}$ vs $\log T$ as shown in Figure 11 . As seen in Figure 11 , the intrinsic thermal resistivity of vanadium varied as $\mathrm{T}^{2}$ for temperatures less than $25 \%$. Our measurements of the temperature dependence of the thermal resistivity are less than the value reported by white and Woods who found that the intrinsic thermal resistivity of vanadium. varied as $\mathrm{T}^{2.4}$ at low temperatures.

\section{Seebeck Coefficient}

The Seebeck coefficient of vanadium is plotted as a function of temperature in Figure 12. The Seebeck coefficient was positive from $10 \mathrm{~K}$ to $240 \mathrm{~K}$ and had a maximum at $70 \mathrm{~K}$. The Seebeck coefficient at temperatures less than $170 \mathrm{~K}$ varied with sample purity and decreased to zero as the temperature approached zero. Our Seebeck coefficient measurements below $10 \mathrm{~K}$ were not sufficiently precise to observe a discontinuous 


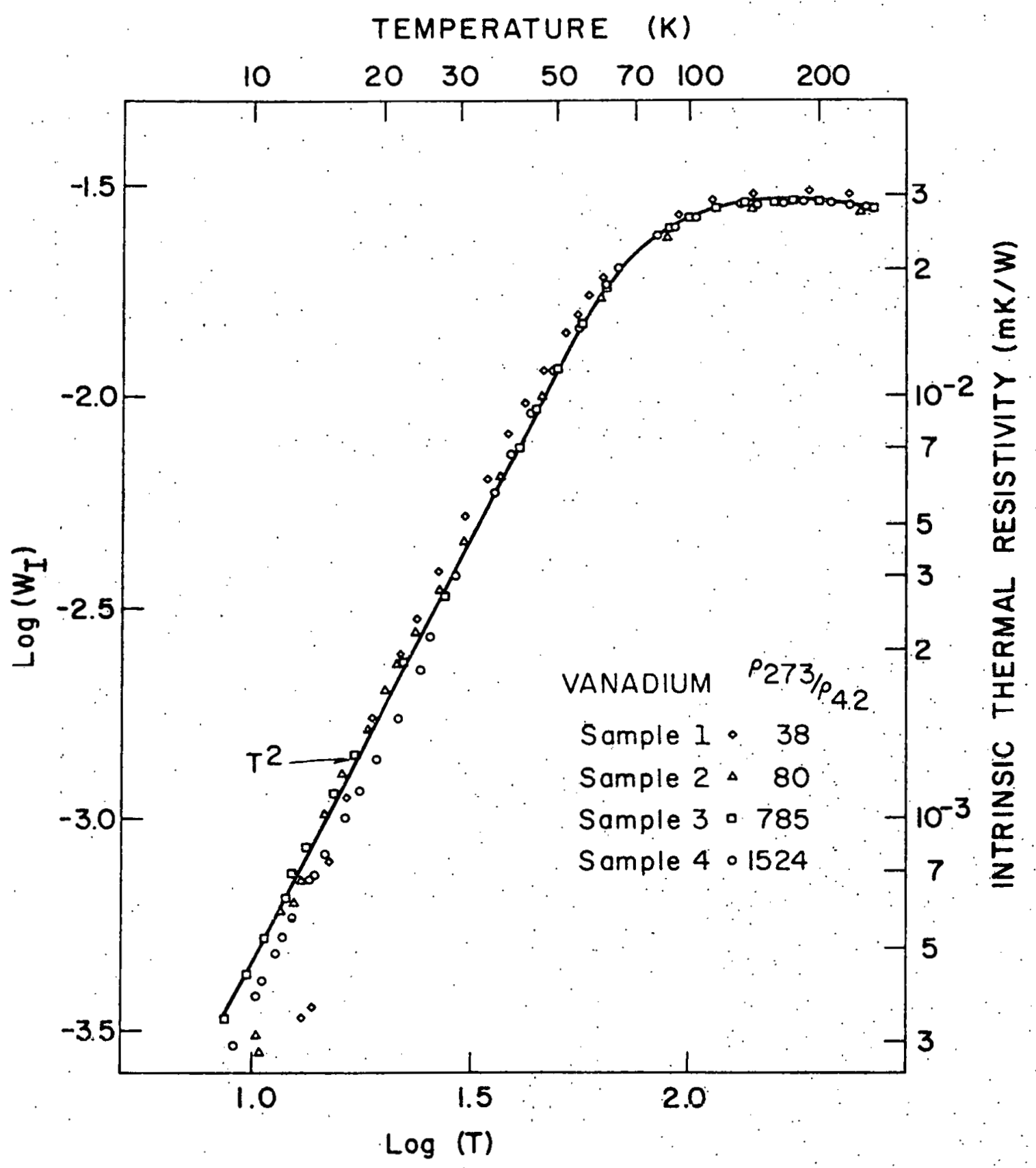

Figure 11: The log of the intrinsic thermal resistivity as a function of the log of the temperature. The intrinsic thermal resistivity varled as $\mathrm{T}^{2}$ from $10 \mathrm{~K}$ to $25 \mathrm{~K}$. 


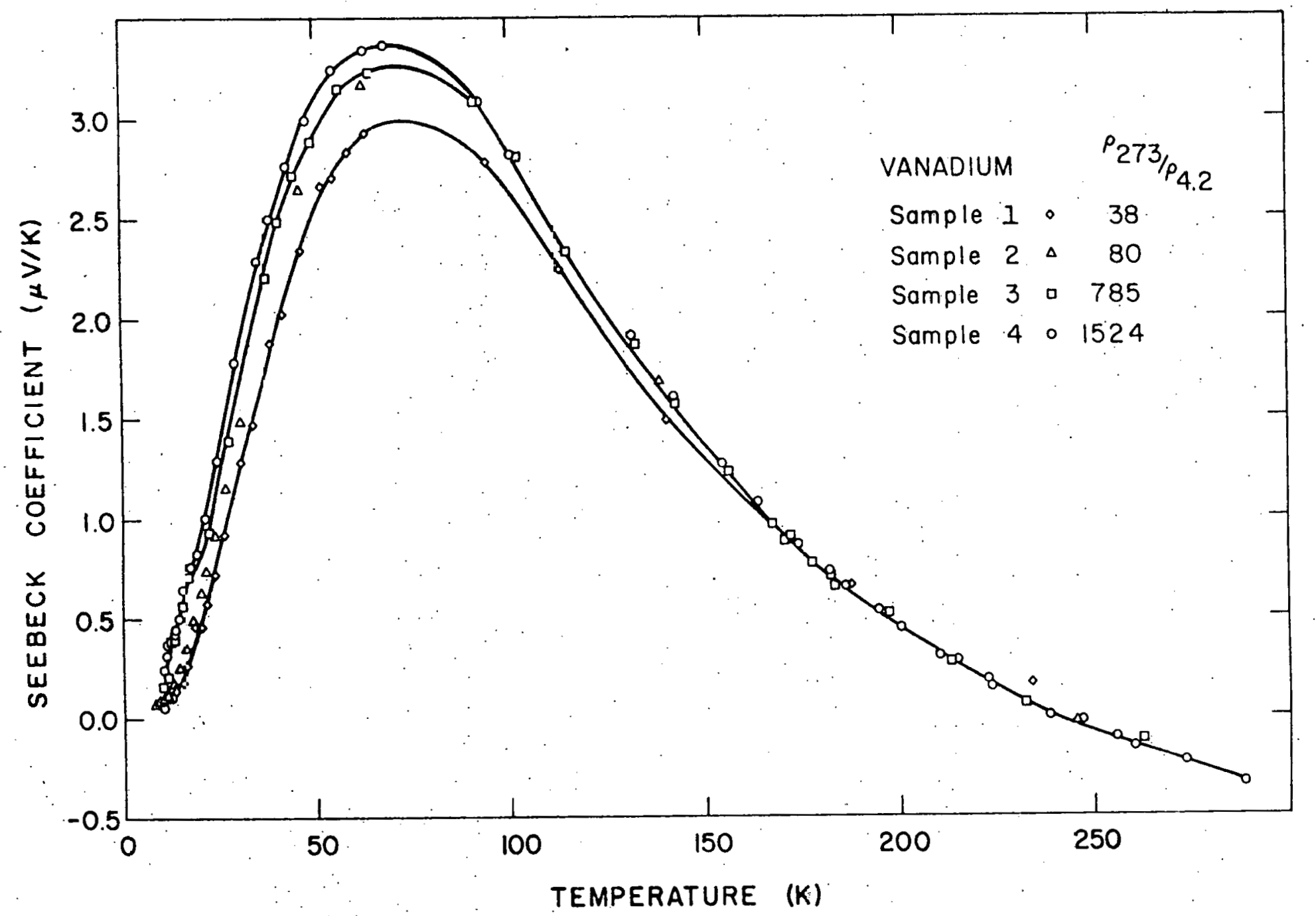

.

Figure 12. The Seebeck coefficient of vanadium as a function of temperature, The Seebeck coefficient varied with sample purity at low temperatures and was positive for temperatures less than $240 \mathrm{~K}$. Above $240 \mathrm{~K}$ the Seebeck coefficient was negative. 
change at the superconducting transition temperature. The highest purity sample, sample 4 , had a Seebeck coefficient maximum of $3.4 \mu \mathrm{V} / \mathrm{K}$ at $70 \mathrm{~K}$. Above $240 \mathrm{~K}$ the Seebeck coefficient of vanadium was negative.

Our Seebeck coefficient results were in agreement with the results of Mackintosh and Sill (23) below $140 \mathrm{~K}$. However above $140 \mathrm{~K}$, our results were in considerable disagreement with the results of Mackintosh and Sill. Mackintosh and Sill reported a temperature dependent hysteresis in the Seebeck coefficient for temperatures above $140 \mathrm{~K}$, depending upon whether the sample was being heated or cooled. In addition it was reported that the Seebeck coefficient decreased anomalously at $217 \mathrm{~K}$ when the sample was being heated and that no discontinuity occurred when the sample was being cooled. Mackintosh and Sill also reported that the Seebeck coefficient had a relative minimum between $240 \mathrm{~K}$ and $260 \mathrm{~K}$ for both heating and cooling and that the Seebeck coefficient rose with temperature for temperatures above $260 \mathrm{~K}$.

We measured the Seebeck coefficient of vanadium from $80 \mathrm{~K}$ to $290 \mathrm{~K}$ by both increasing and decreasing the ambient temperature. No temperature dependent hysteresis was observed, The heating rates were about $200^{\circ} \mathrm{C} / \mathrm{h}$ while the cooling rate was about $50^{\circ} \mathrm{C} / \mathrm{h}$. Our measurements were made at constant ambient temperature and for temperatures above $140 \mathrm{~K}$, we waited at least two hours per data point for the sample to come to thermal equilibrium.

The anomaly reported by Mackintosh and Sill, and possibly the hysteresis also, was most likely due to the presence of hydrogen in 
their sample. No measurement of the hydrogen concentration was reported. However it was reported that gaseous impurities might be present in the ir sample.

\section{Lorenz Ratio}

The Lorenz ratio of vanadium is plotted in Figure 13. The Lorenz ratio did not depend upon the sample form factor measurement because the same thermometer and voltage probe spacing was used in both electrical resistivity and thermal conductivity measurements.

The Lorenz ratio was less than the Sommerfeld value $\left(L_{0}=2.45 \times 10^{-8}\right.$ $v^{2} / k^{2}$ ) over the entire temperature range. At low temperatures the electrical conductivity and thermal conductivity are limited by impurity scattering and as seen in Figure 13 the Lorenz ratio approached $L_{0}$ at low temperatures.

Sample 1 was anomalous at both high and low temperatures. As seen in Figure 13, the Lorenz ratio of sample i was less than the Lorenz ratio of the other three samples at high temperatures. In addition at low temperatures, sample 1 had a Lorenz ratio which was less than the Sommerfeld value. As seen in Figure 13 the Lorenz ratio of vanadium had a minimum at intermediate temperatures owing to inelastic electronphonon interations. The minimum of the highest purity sample occurred at $30 \mathrm{~K}$ and the minima of the other samples occurred at higher temperatures. 


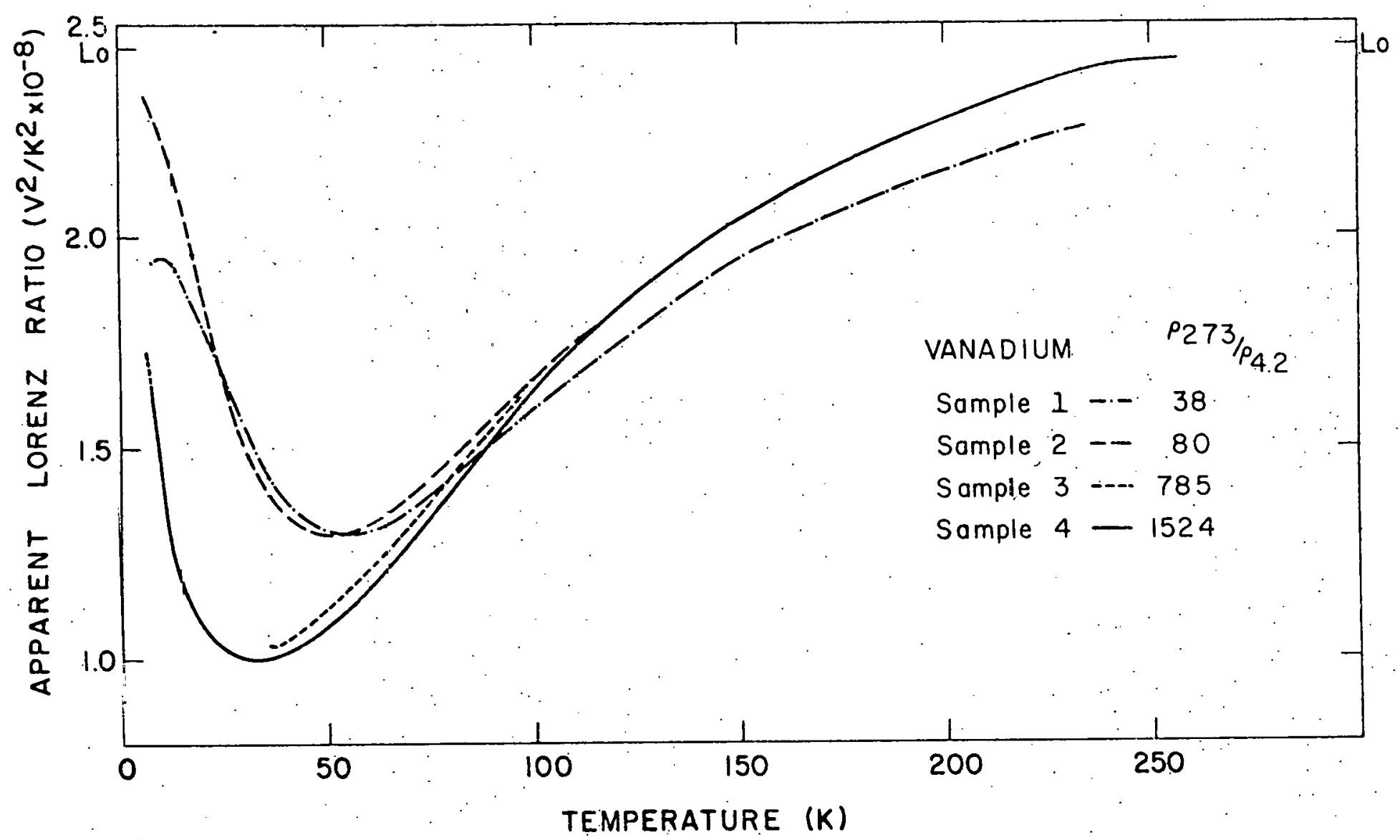

Figure 13. The Lorenz ratio of vanadium plotted as a function of temperature. The Lorenz ratio of vanadium was less than $L_{0}$ over the entire temperature range. 


\section{CHAPTER V:' DISCUSSION}

\section{Thermal Conductivity}

At low temperatures the thermal conductivity of a metal is limited by electron impurity and electron-phonon interactions. The two scattering mechanisms can be separated by applying the thermal analog of Matthiessen's rule to the thermal resistivity.

$$
W(T)=A / T+B T^{2}
$$

where $A / T$ is the thermal resistivity due to electron scattering by point defects and $\mathrm{BT}^{2}$ is the intrinsic thermal resistivity due to electron scattering by phonons: At low temperatures the thermal conductivity of a reasonably pure metal has a maximum at the temperature

$$
\dot{T}=[A / 2 B]^{1 / 3} \text {. }
$$

Figure 14 is a plot of $T W(T)$ vs $T^{3}$ for the four vanadium samples. The $T^{3}=0$ intercept in Figure 14 is the coefficient $A$ and the slope is the coefficient B. The coefficients A and B are listed in Table 4.

As seen in Figure 14 and Table 4, the thermal resistivity of vanadium varied as $A / T+B T^{2}$. Thus at low temperatures the thermal conductivity of vanadium was 1 imited by the scattering of electrons by crystal defects and by normal electron-phonon interactions. However, Matthiessen's rule was not obeyed. The intrinsic thermal resistivity, $B T^{2}$, varied with sample purity, B decreased as the sample purity increased. 


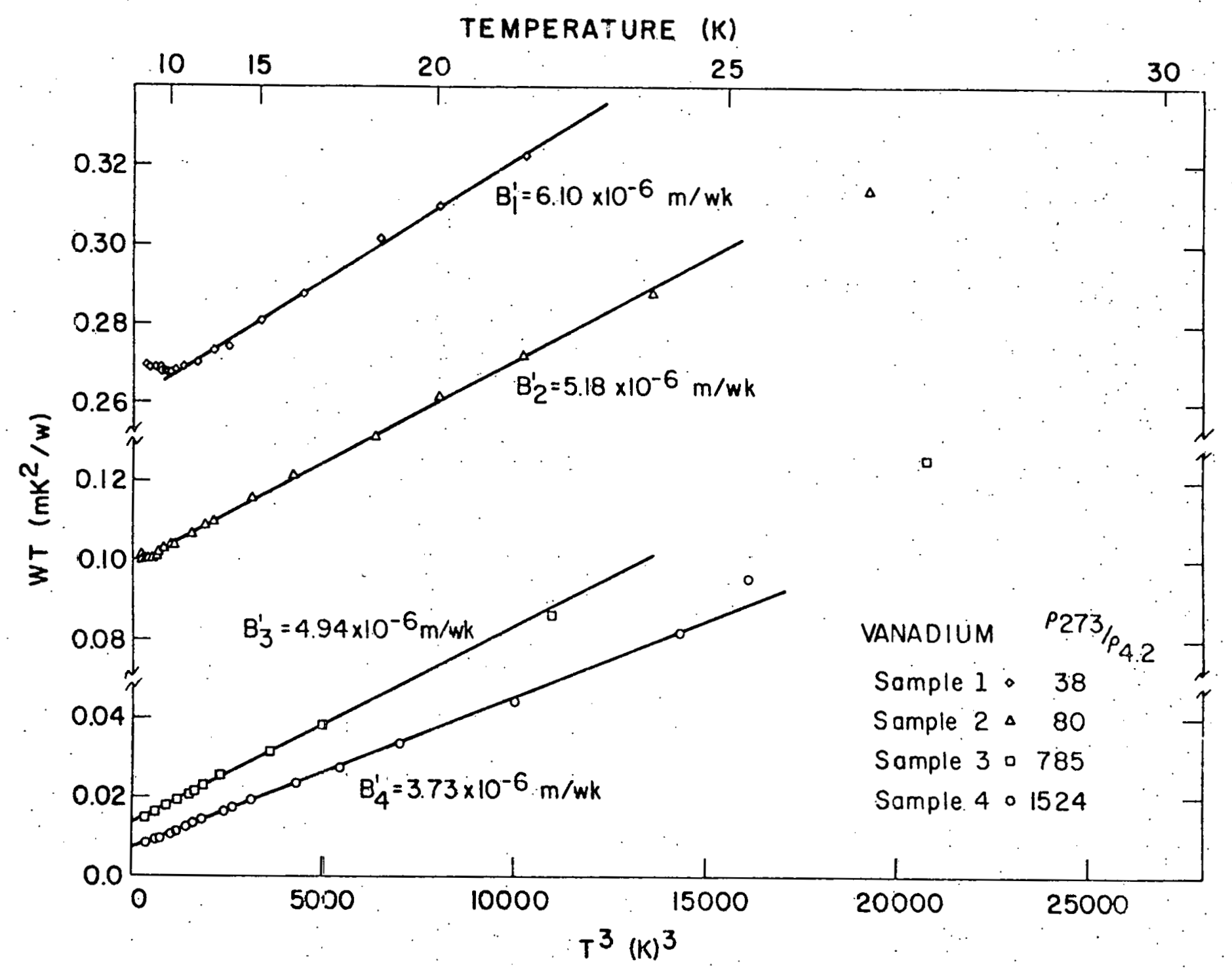

$\stackrel{a}{\omega}$

Figure 14. The thermal resistivity of vanadium times temperature vs. $\mathrm{T}^{3}$. The linear dependence shows that the intrinsic thermal resistivity of vanadium had a $T^{2}$ temperature dependence at low temperatures. 
Table 4. The coefficients $A$ and $B$ of the thermal resistivity of vanadium

\begin{tabular}{ccc}
\hline Sample & $\begin{array}{c}\mathrm{A} \\
\left(\mathrm{mK}^{2} / \mathrm{W}\right)\end{array}$ & $\begin{array}{c}\mathrm{B} \\
\left(10^{-6} \mathrm{~m} / \mathrm{WK}\right)\end{array}$ \\
\hline 1 & 0.269 & 6.10 \\
3 & 0.101 & 5.18 \\
4 & 0.014 & 4.94 \\
& 0.007 & 3.73 \\
\hline
\end{tabular}

A similar variation of $B$ with sample purity has been observed in other metals and most recently in potassium by Newrock and Maxfield (54).

Departures from Matthiessen's rule have been discussed by Bass (55). The thermal resistivity of a metal departs from Mathiessen's rule because the steady state electron distribution for electrons scattered by impurities $\left(f_{i m p}^{o}\right)$ is not equal to the steady state electron distribution for electrons scattered by phonons $\left(f_{\mathrm{Ph}}^{\mathrm{O}}\right)$. The actual steady state electron distribution is a compromise between $f_{i m p}^{0}$ and $f_{P h}^{\circ}$. Consequently the relaxation time for electrons scattered by phonons is affected by the sample purity. Since the intrinsic thermal resistivity depends upon the electron phonon relaxation time, the intrinsic thermal resistivity can also vary with sample purity and Matthiesson's rule is not obeyed.

The magnitude of the low temperature intrinsic thermal resistivity for normal electron-phonon scattering has been calculated by Klemens $(56,57)$. Klemens showed that, 


$$
W_{i}(T)=C\left(\frac{T}{\theta}\right)^{2} \quad W_{i}(\infty)
$$

for temperatures less than $\theta / 20, \theta$ is the Debye temperature and $W_{i}(\infty)$ is the constant thermal resistivity at high temperatures. C is a constant which depends upon the effective number of conduction electrons per atom in the conduction band $\left(\mathrm{N}_{\mathrm{a}}\right)$

$$
C=64\left(N_{a}\right)^{2 / 3}
$$

The highest purity vanadium sample had an intrinsic thermal resistivity which varied as $\mathrm{BT}^{2}$. Thus for the highest purity vanadium sample,

$$
C=B \theta^{2} / W_{i}(\infty)
$$

The Debye temperature of vanadium was $382 \mathrm{~K}$ as determined by Radebaugh and Keesom (12) and $W_{i}(\infty)$ was $2.829 \times 10^{-2} \mathrm{mK} / \mathrm{W}$. Thus for sample 4 , $C=19.2$. The value of $B$ in equation 65 for an ideally pure vanadium crystal would have been less than the value of $B$ for sample 4 since $B$ was impurity dependent. Hence 19.2 is an upper limit for $C$.

Vanadium has a conduction electron configuration of $3 d^{3} 4 s^{2}$. Thus vanadium has two $S$ band conduction electrons per atom and hence should have a valiue of $C$ near 100.

Klemens (57) has shown that low values of $C$ are due to several factors. One reason is that $U$-processes raise the intrinsic thermal 
resistivityat high temperatures above the value predicted by the Wilson theory. Another reason for low values of $\mathrm{C}$ is that the phonon distribution may depart from the Debye model owing to dispersion. In vanadium, the low value of $C$ is most likely due to both dispersion and U-processes. It is difficult to determine which property is affecting the thermal resistivity solely from an analysis of the thermal resistivity.

\section{Electrical Resistivity}

At low temperatures the intrinsic electrical resistivity of a metal should vary as $\mathrm{T}^{5}$ for normal electron-phonon interactions. As discussed earlier, the intrinsic electrical resistivity of vanadium varied as $\mathrm{T}^{3.4}$. At low temperatures the intrinsic electrical resistivity of vanadium was considerably larger than the intrinsic electrical resistivity predicted by the Bloch model. In Figure 15, the intrinsic electrical resistivity of vanadium was compared to the Bloch model by plotting

$$
\rho_{i}(T) / \rho_{B}(T)=\rho_{i}(T) / \rho_{\theta}\left(\frac{T}{\theta}\right)^{5} J_{5}\left(\frac{\theta}{T}\right)
$$

as a function of tempetalure. The ratio in equation 66 was normalized to 1 at high temperatures by setting

$$
\rho_{\theta}=\rho_{i}\left(T_{h}\right) /\left(\frac{T}{\theta}\right)^{5} J_{5}\left(\frac{\theta}{T_{h}}\right) \text {. }
$$

The constant $\rho_{\theta}$ was $1.163 \times 10^{-4} \Omega \mathrm{cm}$ at $297.2 \mathrm{~K}$. The integral $\mathrm{J}_{5}(\theta / \mathrm{T})$ was evaluated by numerically integrating equation 25 with the trapezoidal technique. The Debye temperature was $382 \mathrm{~K}$, 


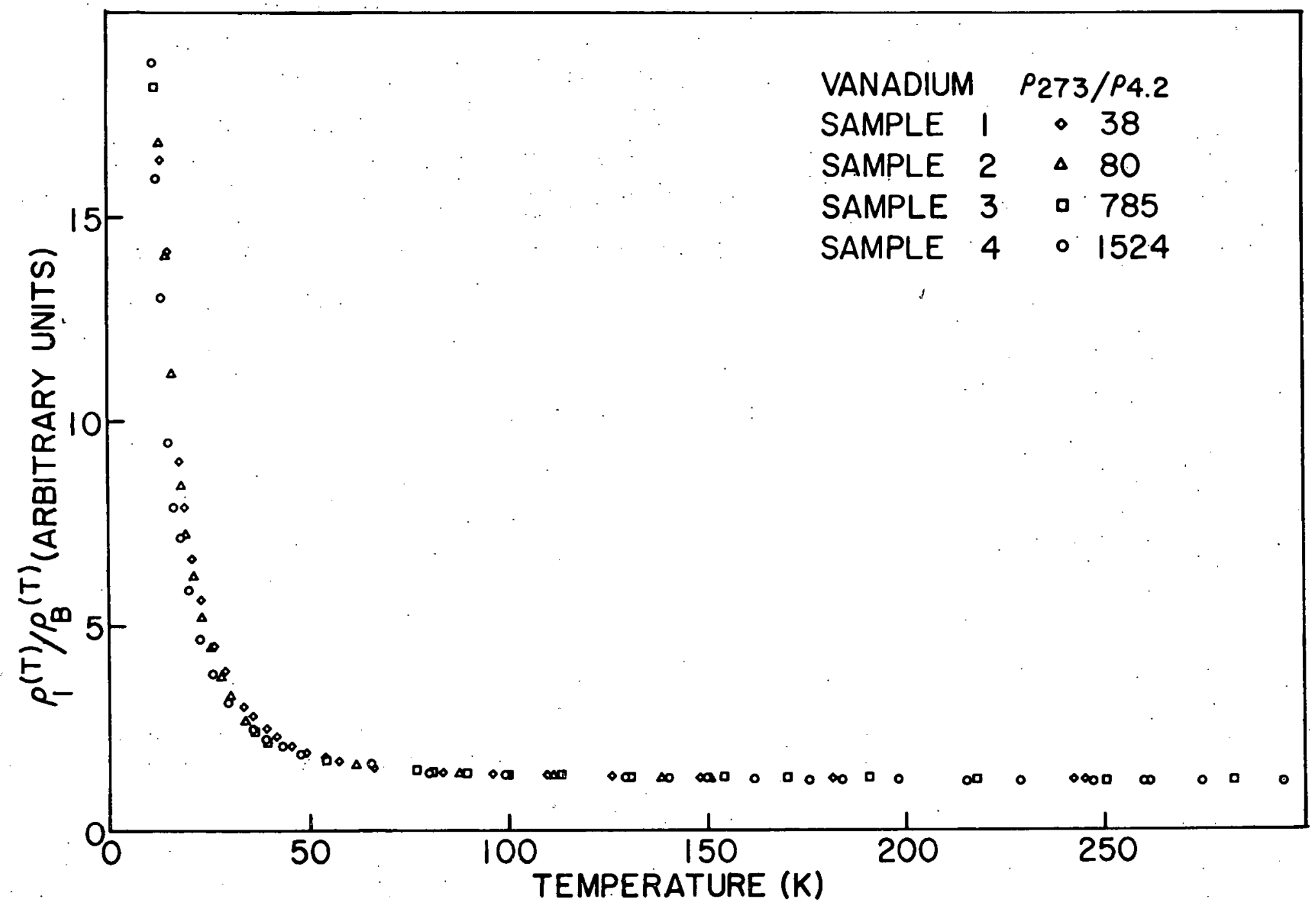

Figure 15. $\rho_{i}(T) / \rho_{B}(T)$ vs $T$ for vanadium, where $\rho_{B}(T)$ is the Block formula. $A b o v e: 70 \mathrm{~K} \rho_{i}(T) / \rho_{B}(T)$ was constant, thus the intrinsic thermal resistivity of vanadium had the temperature dependence predicted by the Block formula. 
As seen in Figure 15, the temperature dependence of $\rho_{i}(T)$ was in good agreement with the Bloch model above $70 \mathrm{~K}$, however at temperatures less than $70 \mathrm{~K}$ the intrinsic electrical resistivity was much greater than the value predicted b.y the Bloch model.

Mott (58) and Mott and Wills (59) have shown that conduction can occur in a transition metal in both the $s$ and $d$ bands.

$$
\sigma=\frac{n_{s} e^{2}}{m_{s}^{*}} \tau_{s}+\frac{n_{d} e^{2}}{m_{d}^{*}} \tau_{d}
$$

where $\mathrm{m}_{\mathrm{s}}^{*}$ and $\mathrm{m}^{*} \mathrm{~d}$ are the electron effective masses of the $\mathrm{s}$ and $\mathrm{d}$ bands and $\tau_{s}$ and $\tau_{d}$ are the $s$ and $d$ band relaxation times. Mott argued that in transition metals the conductivity of $s$ band electrons is much larger than the conductivity of the d band electrons owing to the smaller effective mass of the $s$ band electrons.

Wilson (60) has shown that phonons can scatter electrons from the $s$ to the d band, consequently raising the intrinsic electrical resistivity of a transition metal above the Bloch theory. The d-band acts as a trap into which s electrons are scattered. Wilson has shown that $s$ to $d$ band transitions occur if the phonon wavevector $q$ is larger than the difference in Fermi wavevectors of the $s$ and $d$ band conduction electrons.

$$
q_{M}>\left|\underline{k}_{d}-\underline{k}_{s}\right|
$$

Wilson showed that the intrinsic electrical resistivity of a transition metal was, 


$$
\rho_{i}(T)=\rho_{s s}(T)+\rho_{s d}(T)
$$

where $\rho_{S S}(T)$ is the electrical resistivity of $s$ band electrons (Bloch formula) and $\rho_{s d}(T)$ is the additional electrical resistivity caused by $s$ to $d$ band scattering.

$$
\rho_{s d}(T)=\rho_{s d}^{\prime}\left(\frac{T}{\theta}\right)^{3} \cdot \int_{\theta_{M}^{\prime} / T}^{\theta / T} \frac{e^{z} z^{3} d z}{\left(e^{z}-1\right)^{2}}
$$

where $\rho_{s d}^{\prime}$ is a constant and $\theta_{M}$ is the temperature given by the equation

$$
K_{B} \cdot \theta_{M}=A \omega_{M} \cdot
$$

where $\omega_{M}$ is the frequency of the phonon $q_{M}$. Thus the intrinsic electrical resistivity of a transition metal is

$$
\rho_{i}(T)-4 \rho_{\theta}\left(\frac{T}{\theta}\right)^{5} J_{5}\left(\frac{\theta}{T}\right)+\rho_{s d}^{1}\left(\frac{T}{\theta}\right)^{3}\left(J_{3}\left(\frac{\theta}{T}\right)-J_{3}\left(\frac{\theta M}{T}\right)\right)
$$

At very low temperatures, $J_{3}\left(\frac{\theta}{T}\right) \simeq J_{3}\left(\frac{\theta_{M}}{T}\right)$ and equation 74 reduces to the Bloch equation. At intermediate temperatures equation 74 is larger than the Bloch equation.

Recent band calculations of vanadium by Yasui et al. (61) and Papaconstantopoulos et al. (62) have shown that there are regions of overlap of the bands of vanadium at the Fermi energy. Thus interband transitions can occur for very small q vectors.

If it is assumed that above $5 \mathrm{~K}$, interband transitions are occurring, then at low temperatures the intrinsic thermal resistivity should have a 
$T^{3}$ temperature dependence in addition to the $T^{5}$ temperature dependence. Thus,

$$
\rho_{i}(T)=\rho_{B} T^{5}+\rho_{S \cdot d} T^{3}
$$

where $\rho_{B} T^{5}$ is the electrical resistivity caused by scattering in the $s$ band and $\rho_{s d} T^{3}$ is the electrical resistivity caused by interband scattering. Figure 16 is a plot of $\rho_{i}(T) / T^{3}$ vs $T^{2}$ for three vanadium samples. The intercept in Figure 16 is the coefficient of the $T^{3}$ term of $\rho_{i}(T)$ and the slope is the coefficient of the $T^{5}$ term. As seen in Figure 16 the intercept was non-zero. Thus vanadium had an intrinsic electrical resistivity which varied with temperature as $\rho_{B} T^{5}+\rho_{S d} T^{3}$. At low temperatures $\rho_{O}$ is very nearly equal to $\rho(T)$, thus the scatter at low temperatures in Figure 16 is due to subtracting two nearly equal terms to evaluate $p_{i}(T)$.

As seen in Figure 16 the slope is nearly zero for all three samples. Thus at low temperatures interband scattering is the dominant electron scattering mechanism affecting the electrical resistivity of vanadium. For sample 4 , the cocfficient $\mu_{\text {sd }}$ was $0.274 \times 10^{-11} \mathrm{~s} \mathrm{~cm} / \mathrm{K}^{3}$ and the coefficient $\rho_{B}$ was $0.170 \times 10^{-14} \Omega \mathrm{cm} / \mathrm{K}^{5}$. At $10 \mathrm{~K}$ the interband scattering electrical resistivity was 1600 times as large as the $5-5$ band resistivity. At $20 \mathrm{~K}$ the $\mathrm{s}$ to $\mathrm{d}$ band electrical resistivity is 400 times as large as the $5-s$ band resistivity.

The intrinsic thermal resistivity of a metal with both electronphonon interactions and $s$ to $d$ band scattering is, (63) 


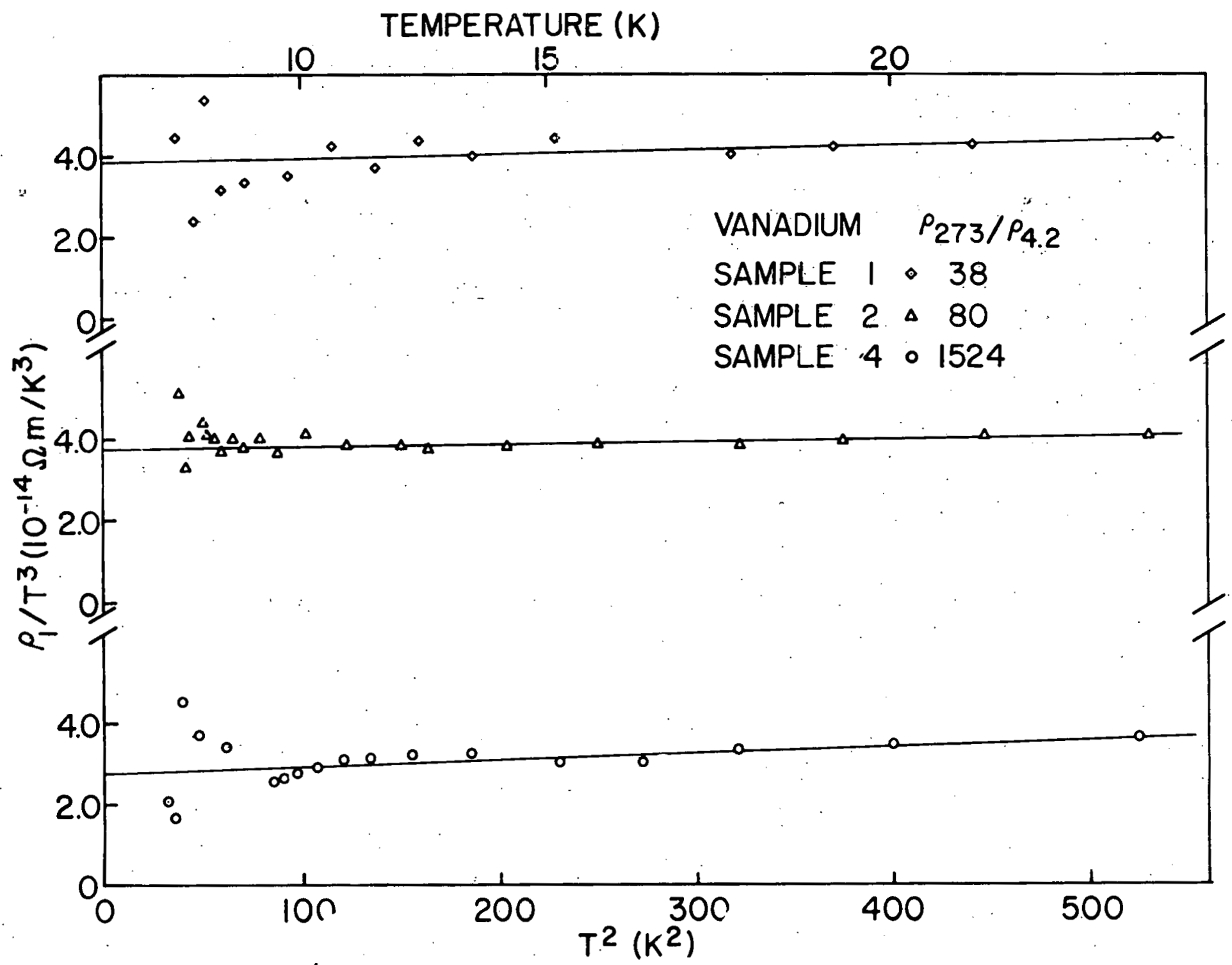

Figure 16. $\rho_{i}(T) / T^{3}$ vs $T^{2}$ for vanadium. $\rho_{i}(T) / T^{3}$ varied linearly with temperature at low temperatures. The large intercepts show that at low temperature the intrinsic thermal resistivity of vanadium had a T3 term. 


$$
W_{i}(T)=W_{S S}(T)+W_{S d}(T)
$$

where $W_{S S}(T)$ is the thermal resistivitycaused by scattering in the $S$ band (Wilson model) and $W_{s d}(T)$ is the additional thermal resistivity due to interband electron scattering. The relaxation times for $s$ to $d$ band electron scattering are the same for electrical and thermal conduction $(33,63)$, thus $W_{s d}(T)$ and $\rho_{s d} T^{3}$ are related by the WFL rule.

$$
W_{s d}(T)=\frac{\rho_{s d}}{L_{0}} T^{2} .
$$

The thermal resistivity caused by interband electron scattering has the same temperature dependence as the thermal resistivity caused by normal electron-phonon interactions (63). Thus at low temperatures,

$$
W_{i}(T)=(B+D) T^{2}
$$

where $B$ is the thermal resistivity caused by normal s band electronphonon interactions and $D$ is the thermal resistivity caused by interband electron scattering.

For sample $4, D=1.12 \times 10^{-6} \mathrm{~m} / \mathrm{WK}$. Thus at low temperatures, interband electron scattering contributed $30 \%$ of the total thermal resistivity.

We have also analyzed the low temperature electrical resistivity fur possible electron-electron scattering. Baber and Wills (64) showed that electron-electron scattering causes the electrical resistivity to 
vary as $T^{2}$ in addition to the $T^{5}$ teinperature dependence for electronphonon interactions. Thus,

$$
\rho_{i}(T)=\rho_{e} T^{2}+\rho_{B} T^{5}
$$

where $\rho_{e} e^{T^{2}}$ is the electrical resistivity due to electron-electron scattering.

The intrinsic thermal resistivity for electron-electron interactions varieslinearly with temperature (33). Thus the intrinsic thermal resistivity of a metal with both electron-electron scattering and normal electron-phonon interactions at low temperatures is

$$
W_{i}(T)=W_{e} T+B T^{2}
$$

where $W_{e e}{ }^{\top}$ is the thermal resistivity due to electron-electron scattering. Herring (65) has shown that the Lorenz ratio for electron-electron scattering is

$$
L_{e}=\frac{\rho}{W_{e e}}
$$

where

$$
L_{e}=1.58 \times 10^{-8} \mathrm{v}^{2} / \mathrm{K}^{2}
$$

Figure 17 is a plot of $\rho_{i}(T) / T^{2}$ vs $T^{3}$ and Figure 18 is a plot of $W_{i}(T) / T$ vs $T$. If electron-electron scattering were causing the additional electrical resistivity at low temperatures then the intercepts of Figures 17 and 18 should be related by the electron-electron WFL rule. As seen in Figure 18, the intercept is zero, thus the thermal resistivity 


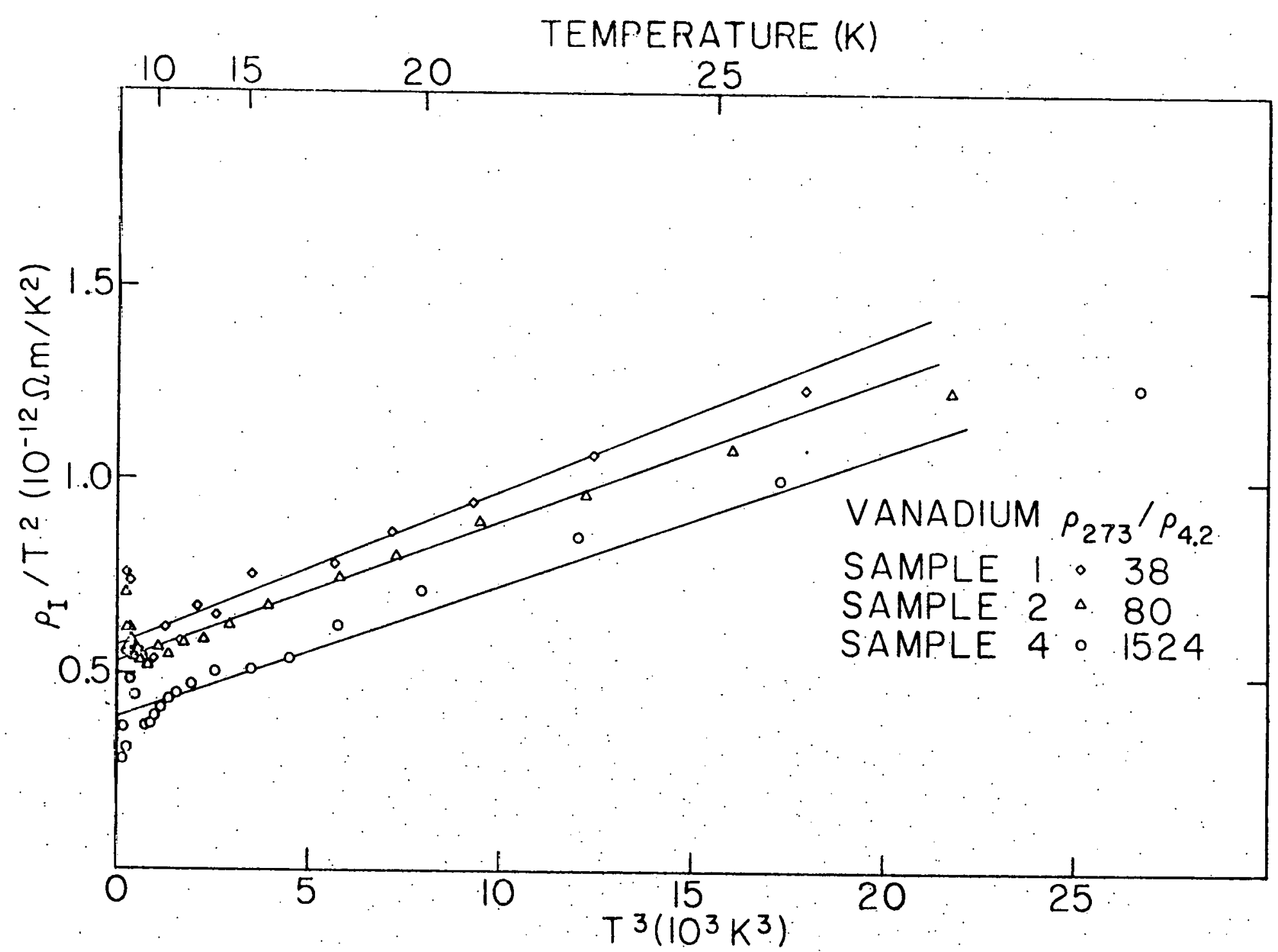

Figure 17. $\rho_{\mathrm{j}}(\mathrm{T}) / \mathrm{T}^{2}$ vs $\mathrm{T}^{3}$ for vanadium. The $\mathrm{T}^{3}=0$ intercept is non-zero showing that the intrinsic electrical resistivity of vanadiun may have a $T^{2}$ term at low temperatures. 


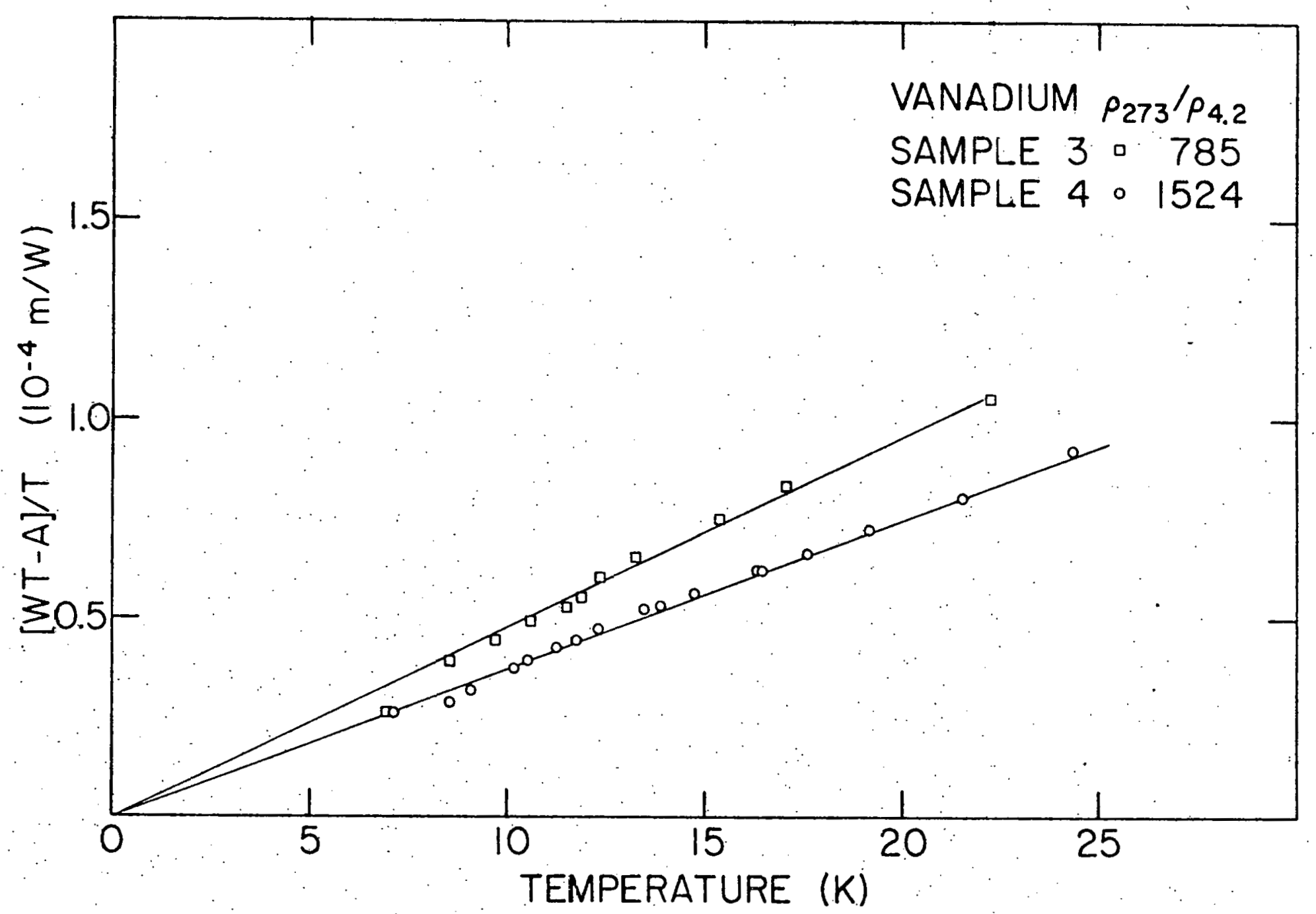

Figure 18. $W_{i}(T) / T$ vs $T$ for vanadium. The $T=0$ intercept is zero. Hence vanadium showed no evidence of low temperature electron-electron scattering. 
showed no evidence of electron-electron interactions at low temperatures. Hence, the excess electrical resistivity of vanadium at low temperatures was not due to electron-electron interactions.

\section{Seebeck Coefficient}

The Seebeck coefficient of a metal has two terms (44),

$$
S(T)=S_{d}(T)+S_{g}(T)
$$

$S_{d}(T)$ is the diffusion Seebeck coefficient and $S_{g}(T)$ is the phonon drag Seebeck coefficient. As discussed earlier the diffusion Seebeck coefficient varies linearly with temperature and has a sign which depends upon the curvature of the Fermi surface in $\underline{k}$ space.

Hana and Sondheimer (66) have shown that the phonon drag Seebeck coefficient resulting from normal phonon-electron interactions has a $\mathrm{T}^{3}$ temperature dependence at low temperatures and a $T^{-1}$ temperature dependence at high temperatures. Bailyn (67) has shown that U-processes give rise to a similar temperature dependence at low and at high temperatures, however, normal phonon-electron processes cause the Seebeck coefficient to be negative and $U$-processes cause the Seebeck coefficient to be positive. At low temperatures, $\theta / 10$, the Seebeck coefficient of a metal should vary as

$$
S(T)=\alpha T+\beta T^{3}
$$

At high temperatures the Seebeck coefficient should vary as 


$$
S(T)=\alpha T+\beta T / T
$$

We have attempted to separate the Seebeck coefficient of vanadium into diffusion and phonon components at low temperatures. The attempt was unsuccessful owing to the large degree of scatter in the Seebeck coefficient data at low temperatures.

Figure 19 is a plot of TS(T) vs $T^{2}$ at high temperatures. In Figure 19 the $T^{2}=0$ intercept was the phonon-drag Seebeck coefficient and the slope was the diffusion Seebeck coefficient. As seen in Figure 19 the plot. was non-linear at high temperatures. MacDonald (44) has pointed out that it is difficult to correctly identify the diffusion and phonontrag Seebeck coefficient in many metals because of competing electron scattering mechanisms. We have found that between $130 \mathrm{~K}$ and $230 \mathrm{~K}$ the Seebeck coefficient of vanadium varied as

$$
S(T)=\alpha^{\prime}+\beta^{\prime} / T .
$$

Figure 20 is a plot of TS(T) vs T. As seen in. Figure 20 TS $(T)$ varied linearly with temperature, $\alpha^{\prime}$ is the slope in Figure 20 and $\beta^{\prime}$ is the $T=0$ intercept. The $\beta ! / T$ term in $S(T)$ was positive at high temperatures owing to electron-phonon U-processes. As discussed earlier the high thermal resistivity at high temperatures also indicates that electronphonon U-processes are important at high temperatures. We are uncertain of our interpretation of $\beta^{1 / T}$ however, since we are unable to explain the origin of the constant term, $\alpha^{\prime}$, in $S(T)$. 


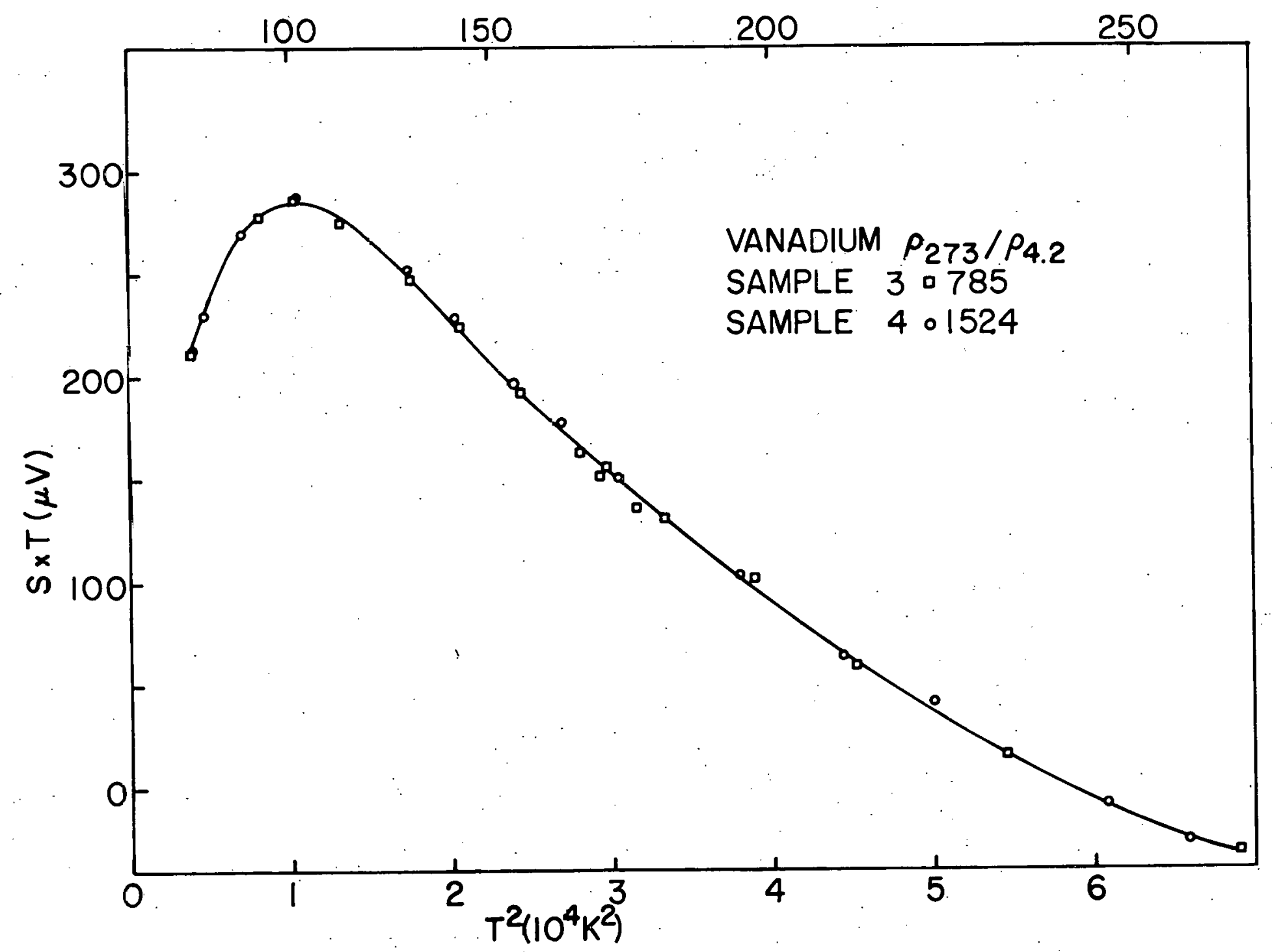

$\infty$

Figure 19. The Seebeck coefficient of vanadium plotted as $T S(T)$ vs $T^{2}$, 


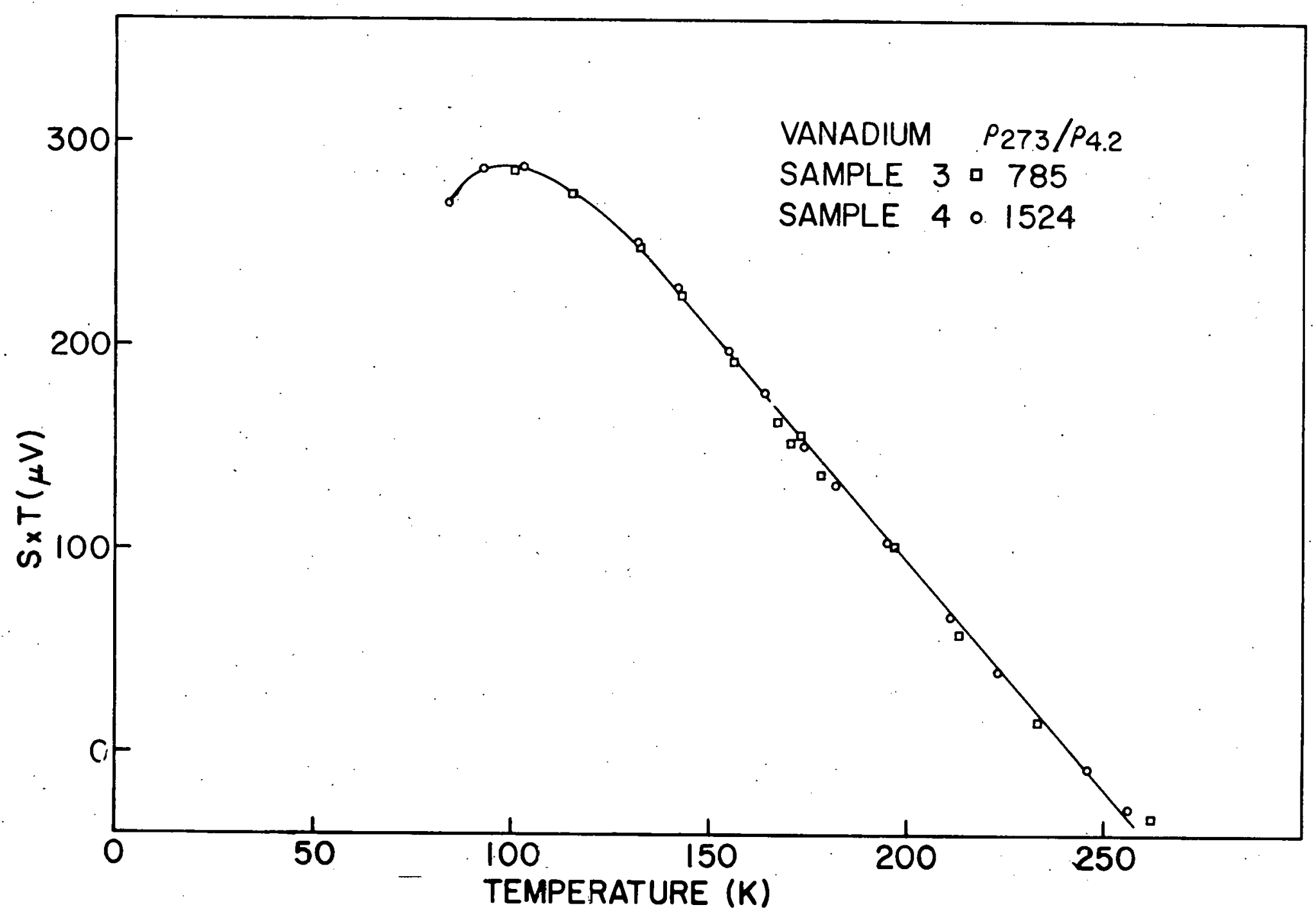

Figure 20. The Seebeck coefficient of vanadium plotted as TS(T) Vs $T$. The linear slope showes that at high temperatures the Seebeck coefficient varied as $\alpha^{\prime}+\beta^{\prime} / T$. 
CHAPTER VI: SUMMARY

Anomalous behavior has been reported in many of the physical properties of vanadium between $180 \mathrm{~K}$ and $250 \mathrm{~K}$. Westlake (24) has shown that the anomalous behavior of the electrical resistivity of vanadium was due to hydrogen in vanadium. We have shown that the electrical resistivity, thermal conductivity and Seebeck coefficient of high purity, hydrogen free vanadium had no anomalous behavior.

At low temperatures the electrical conductivity and thermal conductivity of vanadium were limited by impurity electron scattering and electron-phonon interactions. In addition to the normal s-band electron-phonon interactions, the electrical and thermal conductivities were limited by interband scattering. At low temperatures the electrical resistivity of vanadium was primarily due to interband scattering and interband scalterlng contributed as much as $30 \%$ of the low temperature thermal resistivity. We have observed at low temperatures that Matthiessen's rule was not obeyed.

At high temperatures (above 100K) the thermal resistivily was larger than the value predicted by the wilson theory. In addition we observed a large positive Seebeck coefficient at high temperatures which was due to phonon-drag. The high thermal resistivity at high temperatures and the positive Seebeck coefficient indicated that above $100 \mathrm{~K}$ electron-phonon U-processes were dominant. 


\section{LITERATURE CITED}

1. Carlson, 0, N. and Stevens, E. R., in Kirk-0thmer Encyclopedia of Chemical Technology, edited by A. Standen, John wi ley and Sons, New York, 1970), vol. 21, p. 157.

2. McKechnie, R. K. and Seybolt, A. U., J. Electrochem. Soc. 97, 311 (1970).

3. Beard, A. P. and Crooks,. D. D., J. Electrochem. Soc. 101, 597 (1954).

4. Carlson, 0. N. and Owen, C. V., J. Electrochem. Soc. 108, 88 (1961).

5. Sullivan, T. A., J. Metals 17, 45 (1965).

6. Lei, K. P. V. and Sullivan, T. A., J. Less-Common Metals 14, 145 (1966).

7. Reed, R. E., J. Vac. Sci. Technol. I, S105 (1970).

8. Carlson, 0. N., Schmidt, F. A. and Alexander, D. G., Met. Trans. 3, 1249 (1972).

9. Worley, R. D., Zemansky, M. W. and Boorse, Phys. Kev. 9y, 447 (1955).

10. Corak, W. S., Goodman, B. B., Satterthwaite, C. B. and Wexler, A., Phys. Rev. 102, 656 (1956).

11. Wexler, $\Lambda$. and Corak, W. S., Phys, Rev. $\underline{85}, 85$ (1952).

12. Radebaugh, R. and Keesom, P. H., Phys. Rev. 149, 209 (1966).

13. Bolef, D. I., Smith, R. E. and Miller, J. G., Phys. Rev. B 3,4100 (1971).

14. Alers, G. A., Phys. Rev. 119, 1532 (1360).

15. Burger, J. P. and Taylor, M. A., Phys. Rev. Lett. 6, 185 (1961).

16. Loomis, B. A. and Carlson, 0. N. in Reactive Metals, edited by W. R. Clough, (Interscience Publishers, New York, 1958), vol. 2, P. 227. 
17. Rastoker, W. and Yamamoto, A. S., Trans. Am. Soc. Metals 47, 1002 (1955).

18. Smirnov, Y. M. and Finkel, V. A., Soviet Phys. JETP 22, 756 (1966).

19. White, G. K. and Woods, S. B., Phil. Trans. R. Soc. 251, 273 (1959).

20. Suzuki, Haruhiko, Minomura, S. and Miyahara, S., J. phys. Soc. Japan 21, 2089 (1966).

21. Bolef, D. I., de Klerk,' J. and Brandt, G. B., Bull. Am. Phys. Soc. I. 236 (1962).

22. Suzuki, Haruhiba and Miyahara, S., J. phys. Soc. Japan 20, 2102 (1965).

23. Mackintosh, A. R. and Sill, L., J. Phys. Chem Sol ids 24, 501 (1963).

24. Westlake, D. G., Trans. metall. Soc. A.I.M.E. 239, 1341 (1967).

25. Westlake, D. G., Phill. Mag. 16, 905 (1967).

26. Chiba, T. and Takano, S., J. phys. Soc. Japan 31, 1113 (1971).

27. Rosenberg, H. M., Trans. Roy. Soc. A 247, 441 (1955).

28. Klemens, P. G., in Thermal Conductivity, edited by R.P. Tye, (Academic Press, New York, 1969), vol. 1, p. 1.

29. Klemens, P. G., in Handbuch der Physik, edited by S. Flügge, (Springer-Verlag, Berlin; 1956), vol.14, p. 198.

30. Klemens, P. L., in Solid State Physics, edited by F. Seitz and D. Turnbul1, (Academic Press, New York, 1958), vol. 7, P. 1.

31. Mendelson, K., and Rosenberg, H. M., in Solid State Physics, edited by F. Seitz and D. Turnbull, (Academic Press, New York, 1961), vol. 12, p. 223.

32. Ziman, J. M., Electrons and Phonons, (Oxford University Press, London, 1960).

33. Wilson, A. H., The Theory of Metals, (Cambridge University Press, London, 1953).

34. Peierls, R. Quantum Theory of Solids, (Clarendon Press, Oxford, 1955). 
35. Klemens, P. G., Aust. J. Phys. Z, 70 (1954).

36. Klemens, P. G. and Jackson, J. L., Physica 30, 2031 (1964).

37. Bloch, F., Z. Phys. 59, 208 (1930).

38. Jones, H. in Handbuch der Physik, edited by S. Flügge (SpringerVerlag, Berlin, 1956) vol. 19, p. 227.

39. Kohler, M. Z. Phys. 124, 772 (1948).

40. Kohler, M. Z. Phys. 125, 679 (1949).

41. Sondheimer, E. H., Proc. Roy. Soc. A 203, 75 (1950).

42. Wilson, A. H., Proc. Camb. Phil. Soc. 33, 371 (1937).

43. Zimon, J. M., Proc. Roy. Soc. A 226, 436 (1954).

44. MacDonald, D. K. C., Thermoelectricity: an introduction to the principles, (John Wiley and Sons, Inc., New York, 1962).

45. Peterson, D. T. and Schmidt, F. A., J. Less-Common Metals 24, 223 (1971).

46. Peterson, D. T., Schmidt, F. A. and Verhoeven, J. D., Trans. metall. Soc. A.I.M.E. 236, 1311 (1966).

47. Schmidt, F. A., Conzemius, R. J., Carlson, 0. N. and Seve, H. J., Anal. Chem. $46810(1974)$ :

48. White, G. K., in Thermal Conductivity, edited by R. P. Tye, (Academic Press, New York, 1969), vol. 1, p: 69.

19. Jung, W. D., is Repurt 3631 (1975).

50. Corrunccini, R. J., Chem. Eng. Prog. $53 \underline{262}$ (1957).

51. Christian, J. W., Jan, J. P., Pearson, W. B. and Templeton, I. M., Proc. Roy. Soc. A 245, 213 (1958).

52. Hust, J. G. and Sparks, L. L., NB̈S Report 9771, (1970).

53. Husl, J. G. and Sparks, L. L., NBS Special Publication 260-3i, (1971).

54. Newrock, R. S. and Maxfield, B. W., Phys. Rev. BI, 1283 (1972).

55. Bass, J., Adv. Phys. 21, 431 (1972). 
56. Klemens, P. G., Aust. J. Phys. 7, 64 (1954).

57. Klemens, P. G., Aust. J. Phys. I, 70 (1954).

58. Mott, N. F., Proc. Phys. Soc. 47, 571 (1935).

59. Mott, N. F. and Wills, H. H., Proc. Roy. Soc. A 153, 699 (1936).

60. Wilson, A. H., Proc. Roy. Soc. A 167, 580 (1938).

61. Yasui, M., Hayashi, E. and Shimizu, M., J. phys. Soc. Japan 29, 1446 (1970).

62. Papaconstantopoulos, D., Anderson, J. and McCaffrey, J., Phys. Rev. BE, 1214 (1972).

63. Kemp, W. R. G., Klemens, P. G., Sreedhar, A. K. and White, G. K., Proc. Phys. Soc. A67, 728 (1954).

64. Baber, W. G. and Wills, H. H., Proc. Roy. Soc. A 158, 383 (1937).

65. Herring, C., Phys. Rev. Letters 19, 167, 684(E) (1967).

66. Hana, 1. 1. and Sondheimer, E. H., Proc. Roy. Soc. A 239, 247 (1957).

67. Bailyn, M., Phys. Rev. 112, 1587 (1958).

68. Ries, R. P. and Moore, K. B., Rev. Sci. Inst. 41, 996 (1970).

69. Bevington, R. P., Data Reduction and Error Analysis for the Physical Sciences, (McGraw Hill, New York, 1969). 


\section{ACKNOWLEDGMENTS}

It is with pleasure that 1 thank my major professor Dr. G. C. Danielson for his encouragement and guidance throughout this research. 1 also thank $P$. Sidles and $H$. Shanks for the assistance they gave me in this research. 0 . Sevde provided technical assistance for which 1 am very grateful. Dr. B. F. T. Bolker worked with me in the design of the apparatus. F. Schmidt kindly provided the samples and R. Conzemius did the SSMS analysis. I also thank M. Anderson for calibrating the germanium thermometers. 


\section{APPENDIX A: SHIELD DIFFERENTIAL TEMPERATURE CONTROLLER}

The shield-sample differential temperature controller holds the temperature of the shield gradient heater at the same temperature as the sample gradient heater. A differential thermocouple is mounted between the sample gradient heater and the shield gradient heater. This thermocouple is the temperature sensor for the temperature controller.

The thermocouple EMF is amplified by a Keithley $149 \mu \mathrm{V}$ meter. The output of the Keithley $(-10 \mathrm{~V}$ to $+10 \mathrm{~V})$ is fed into the temperature controller, Figure 21. The controller outputs current in either manual or automatic mode. When the controller is in the "Manual" mode the output current is increased or decreased by the two respective push button switches. These switches increase or decrease the output of op. amp. I by supplying either $+15 \mathrm{~V}$ or $-15 \mathrm{~V}$ to the $(-)$ input of op. amp. 1 . When the mode switch is in the "automatic" position ap. amp. I samples the output voltage of the Keithley $149 \mu \mathrm{V}$ meter and swings positive or negative with a time constant determined by the "rate" potentiometer. Op. amp. 2 amplifies the output of op. amp. I with a gain determined by the "gain" potentiometer. The output current of op, amp. 2 is amplified by the transitors which provide current for the shield heater.

In order to control temperature oscillations when the controller is turned on an "approach" control has been incorporated into the controller. The output of op. amp. I will only decrease when the Keithley $149 \mathrm{drives}$ the (+) input of op. amp. 2 negative. As the shield temperature approaches 
that of the gradient heater the output of the Keithley decreases. The voltage into the $(+)$ input of op. amp, 2 decreases, consequently its. output voltage decreases, decreasing the current supplied to the shield heater. 


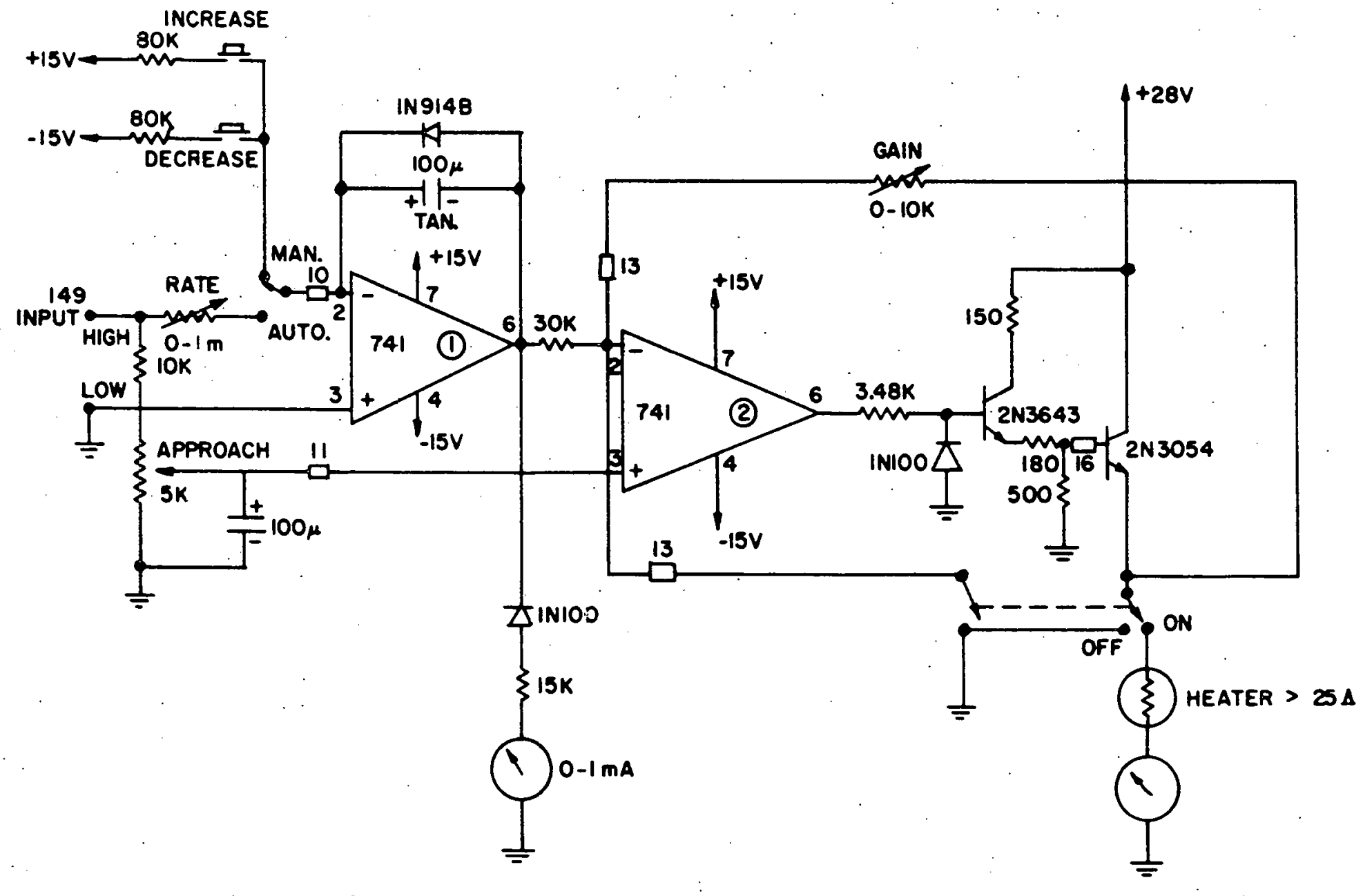

Figure 2‡. D.C. proportional temperature controller. 


\section{APPENDIX B: A.C. RESISTANCE TEMPERATURE CONTROLLER}

The a.c. temperature controller was built following a design by Ries et al. (68). The controller passes an a.c. current through a resistance thermometer which is the temperature sensor. The resistance thermometer is in series with a reference resistor. The two resistors are the two arms of four-arm a.c. bridge. The other two arms are the two primary windings of a toroidal transformer. The two primary windings of the transformer are wound in a bifilar manner such that when the two primary currents are equal, the secondary current of the transformer is zero. In the temperature controller bridge the resistance thermometer is in series with one primary of the transformer. The other primary is in series with the reference resistor. The $530 \mathrm{~Hz}$ oscillator supplies current through the resistance thermometer (sensor) and one transformer primary and through the reference resistor and second transformer primary as shown in Figure 22. The impedence of the two transformer primaries are nearly equal, hence the secondary output of the transformer is an a.c. voltage which is amplitude and phase sensitlve to the difference in impedence between the resistance thermometer and reference resistor.

The transformer was wound on a ferrite core purchased from Ferroxcube corp. 1 The primary was wound with ten bifilar turns of \#30 copper wire and the secondary was wound with 1000 turns of \#38 copper wire. The transformer was mounted in a temperature controlled copper oven that was

\footnotetext{
Ferroxcube, K3-005-01, Ferroxcube Corp. of America, Saugerties, N. Y. .
} 


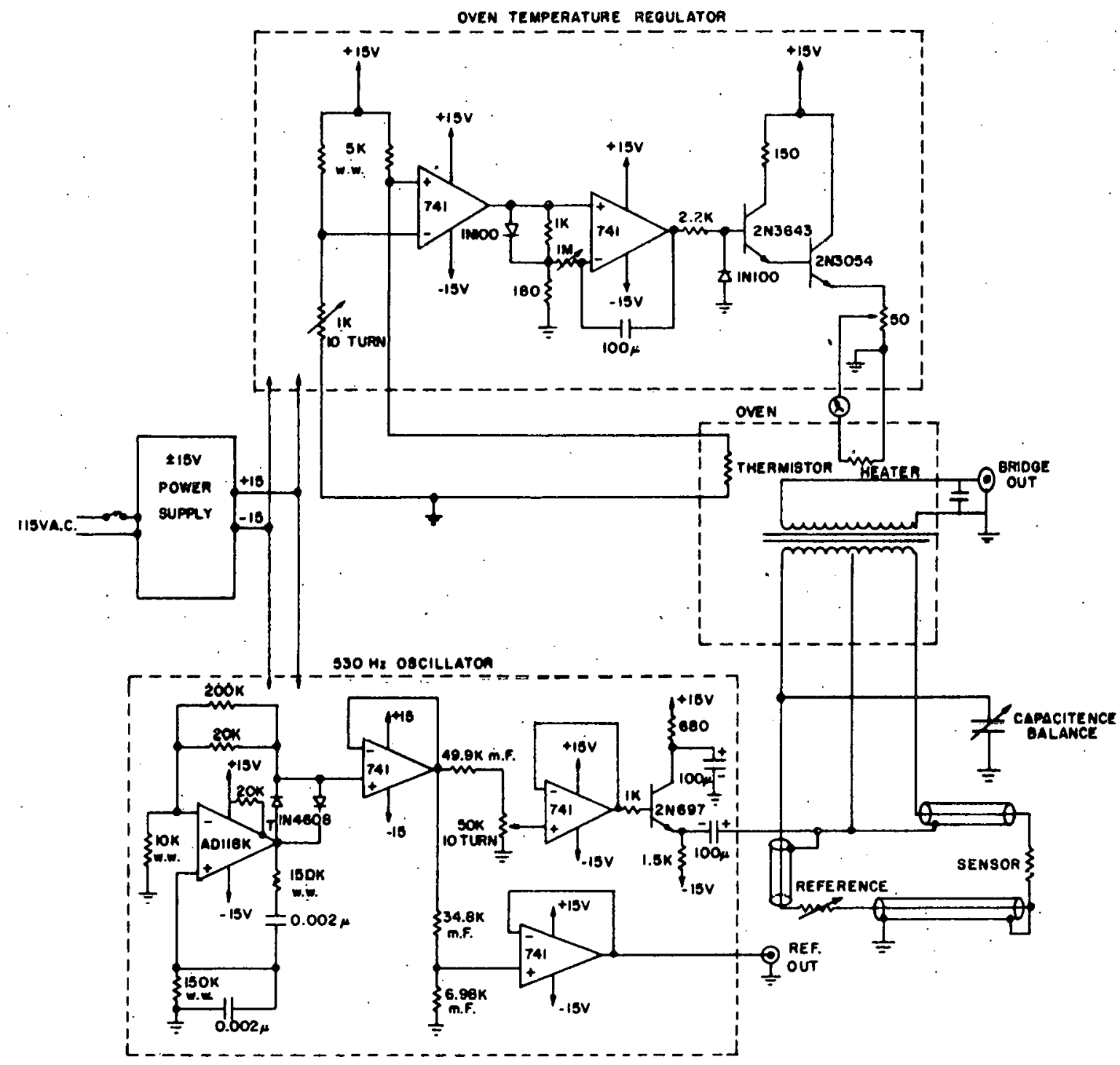

Figure 22. A.C. resistance temperature controller bridge circuit. 
electrostatically and magnetically shielded. The oven temperature was controlled by a d,c. temperature controller shown in figure 22.

The $530 \mathrm{~Hz}$ bridge oscillator shown in Figure 22 was designed by Wayne Rhinehart at Ames Laboratory.

The output of the bridge is amplified by the lock-in amplifier shown in the block diagram in Figure 23. The lock-in amplifier, which was built by the instrument group at Ames Laboratory, produces a $\pm 0.5 \mathrm{~V}$ d.c. signal which is polarity and magnitude sensitive to the phase and amplitude of the output of the bridge.

The output of the lock-in amplifier is fed to a Leeds and Northrup series 80 temperature controller. The zero to $5 \mathrm{~mA}$ output of the $L \& N$ temperature controller is amplified by the d.c. amplifier shown in Figure 24 and supplies the sample holder heater current.

The temperature controller bridge-lock-in amplifier network can detect resistance changes of the resistance thermometer $(\Delta R / R)$ of $10^{-5}$. The resistance thermometer was a $2 \mathrm{~W}, 1000 \Omega$ carbon resistor. The outer bakelite casing of the resistor was sanded off in order to improve the thermal responce time of the resistor. The resistor had a resistance of $1000 \Omega$ at $280 \mathrm{~K}, 1800 \Omega$ at $77 \mathrm{~K}$ and $40 \mathrm{~K} \Omega$ at $5 \mathrm{~K}$. Thus the temperature controller was sensitive to temperature changes of $0.05 \mathrm{mK}$ at $5 \mathrm{~K}, 1.8 \mathrm{mK}$ at $77 \mathrm{~K}$ and $12 \mathrm{mK}$ at $280 \mathrm{~K}$. 


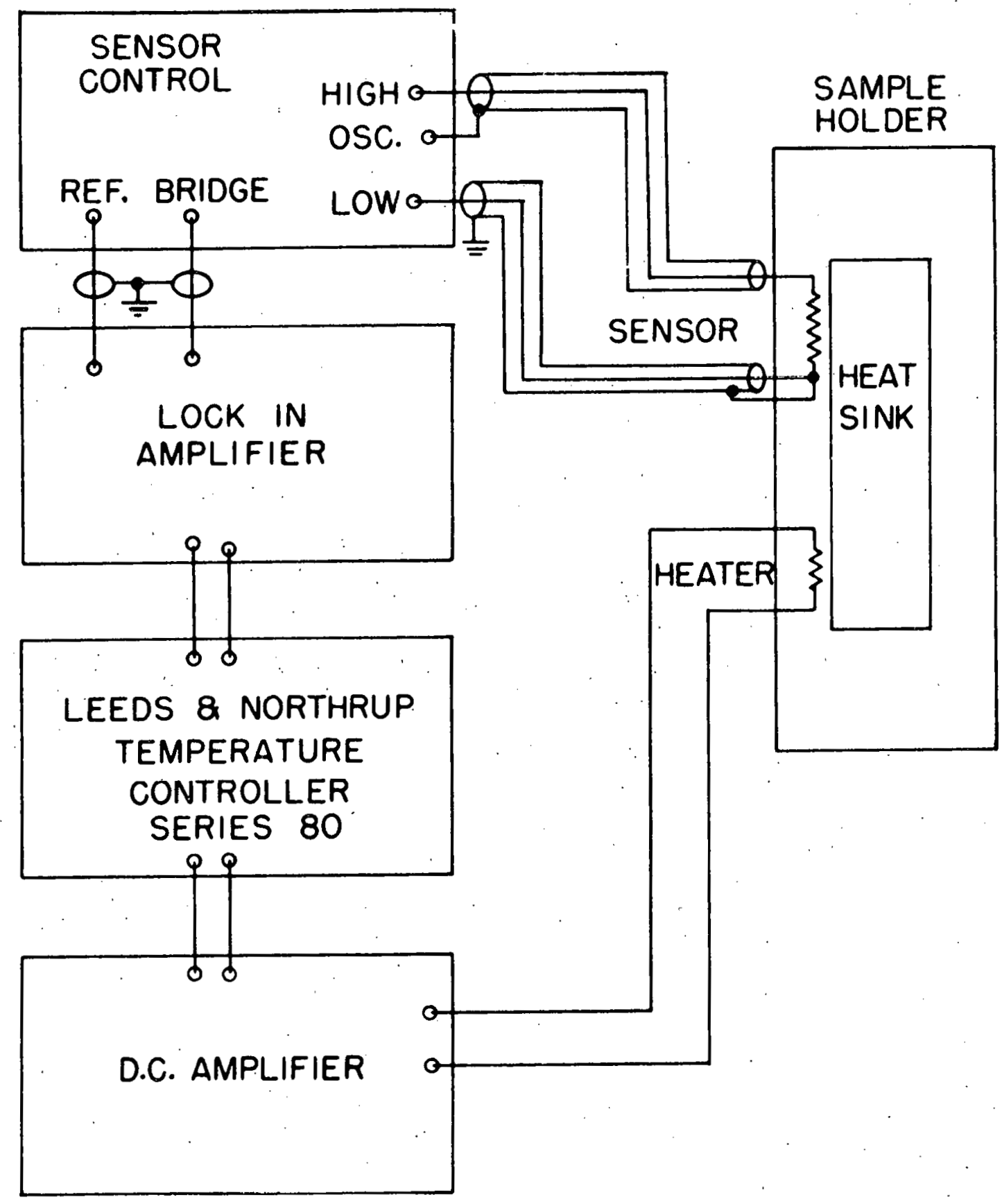

Figure 23. Block diagram of the a.c. temperature controller. 


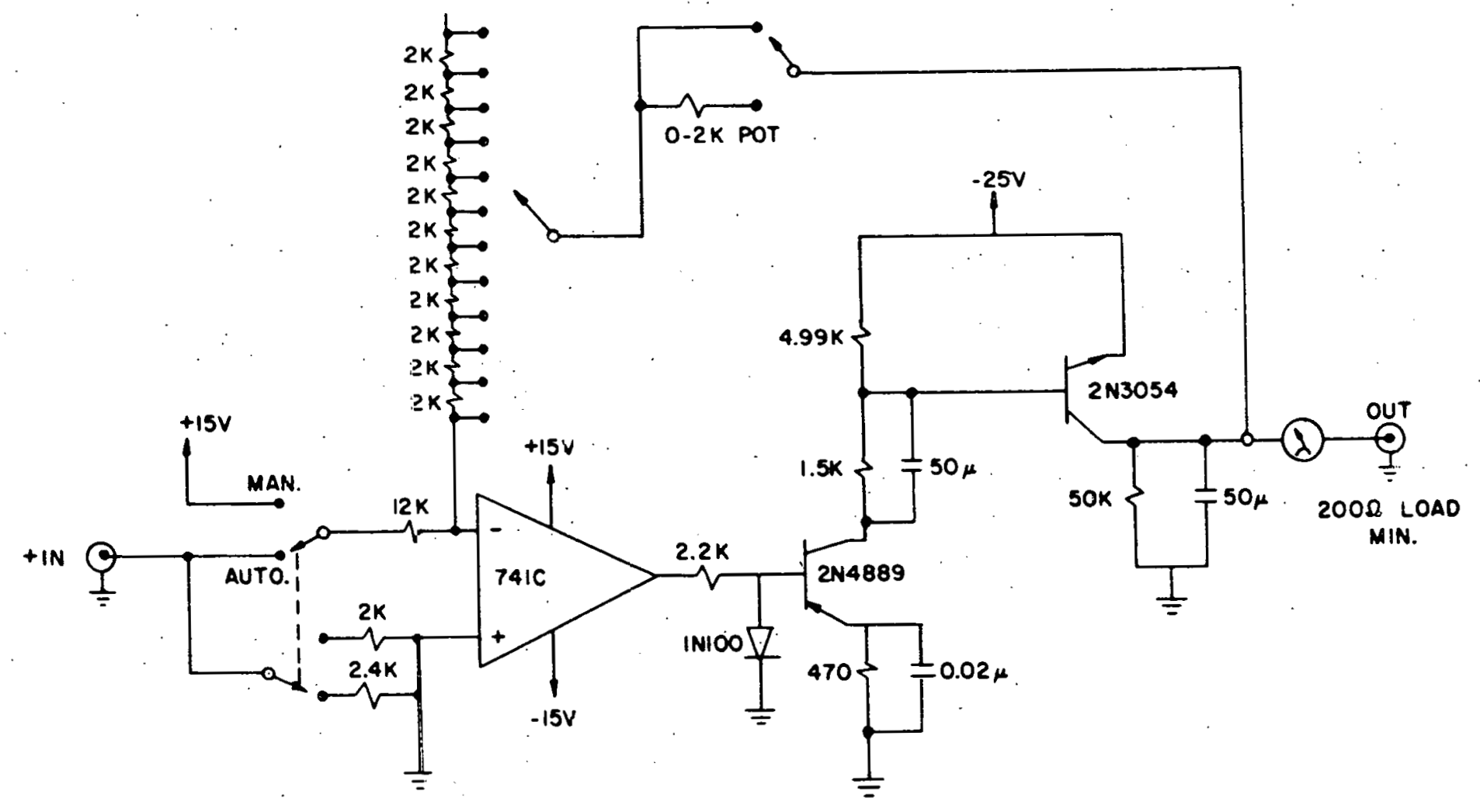

Figure 24. The d.c. amplifier circuit. 
APPENDIX C: THERMOCOUPLE CALIBRATION AND ERROR ANALYSIS

The absolute thermocouple was calibrated by measuring the bath reference temperature $T_{B}$, the thermocouple temperature $T$ and the thermocouple voltage V. 44 data points were taken for $5.3 \mathrm{~K}<\mathrm{T}<72 \mathrm{~K}$ and 34 data points were taken for $77 \mathrm{~K}<\mathrm{T}<314 \mathrm{~K}$. These data were least squares fitted to three polynomials of ninth degree over the temperature ranges: $5.3 \mathrm{~K}<\mathrm{T}<18.96 \mathrm{~K}, 13.1 \mathrm{~K}<\mathrm{T}<72 \mathrm{~K}$ and $77 \mathrm{~K}<\mathrm{T}<314 \mathrm{~K}$. The data were fitted to

$$
V=\sum_{i=1}^{9} A_{i}\left[T_{B}^{i}-T^{i}\right]
$$

The standard deviation of the three fits were:

$$
\begin{array}{lll}
5.22 \times 10^{-4} \mathrm{mV} & \text { : for } & 5.3 \mathrm{~K}<\mathrm{T}<18.9 \mathrm{~K} \\
5.57 \times 10^{-4} \mathrm{mV} & \text { for } & 13 \mathrm{~K}<\mathrm{T}<72 \mathrm{~K} \\
1.29 \times 10^{-3} \mathrm{mV} & \text { for } & 77 \mathrm{~K}<\mathrm{T}<314 \mathrm{~K}
\end{array}
$$

The uncertainty in the calculation of the coefficients $A_{i}$ is (69)

$$
\sigma_{A_{i} A_{j}}=s_{v}^{2} \varepsilon_{i j}
$$

where $\varepsilon_{i j}$ is the least squares error matrix and the variance $S_{V}^{2}$ is

$$
S_{V}^{2}=\frac{1}{N-9} \sum_{\ell=1}^{N}\left[V_{\ell}-f\left(T_{\ell}\right)\right]^{2} .
$$

$N$ is the number of data points and $f\left(T_{l}\right)$ is the polynomial defined in 
equation 86. The least squares, fitting has an uncertainty $\sigma_{F}$, where

$$
\begin{aligned}
\sigma_{F}^{2} & =\sum_{i=1}^{9} \sum_{j=1}^{9} \sigma_{A_{i} A_{j}}^{2}\left[\frac{\partial V}{\partial A_{i}}\right]\left[\frac{\partial V}{\partial A_{j}}\right] \\
& =s_{V}^{2} \sum_{i=1}^{9} \underset{j=1}{g} \varepsilon_{i j}\left[T_{B}^{i}-T^{i}\right]\left[T_{B}^{j}-T^{j}\right] .
\end{aligned}
$$

$\sigma_{F}$ is the uncertainty in calculating $V$ at temperature $T$ with equation 86.

$\sigma_{F}$ is non-zero because there is an uncertainty in measuring the data $\left[V, T ; T_{B}\right]$. Consequently there is an uncertainty in calculating the coefficients $A_{i}$.

Temperature wasmeasured with the thermocouple by measuring the bath temperature $T_{B}$ and the Seebeck voltage $V$. The bath temperature was measured with an uncertainty of $\sigma_{\Gamma_{B}}=2 \mathrm{mK}$ and the Seebeck voltage was measured with an uncertainty of $\sigma_{V}=0.5 \mu \mathrm{V}$. The temperature $T$ was calculated by iteration of equation 86 . The error in the temperature measurement is (69),

$$
\sigma_{T}^{2}=\sigma_{T_{B}}^{2}\left(\frac{\partial T}{\partial T_{B}}\right)^{2}+\sigma_{V}^{2}\left(\frac{\partial T}{\partial V}\right)^{2}+\sum_{i=1}^{9} \sum_{j=1}^{9} \sigma_{A_{i} A_{j}}^{2}\left(\frac{\partial T}{\partial A_{i}}\right)\left(\frac{\partial T}{\partial A_{j}}\right)
$$

The partial derivatives are evaluated from equation 86 ,

$$
\frac{\partial T}{\partial T_{B}}=\frac{\stackrel{9}{i}{ }_{i} A_{i} i T_{B}^{i-1}}{S(T)}=\frac{S\left(T_{B}\right)}{S(T)}
$$




$$
\begin{aligned}
& \frac{\partial T}{\partial V}=\frac{1}{S(T)} \\
& \frac{\partial T}{\partial A_{i}}=\frac{\left[T_{B}^{i}-T^{i}\right]}{S(T)}
\end{aligned}
$$

where

$$
S(T)=\sum_{i=1}^{9} A_{i} i T^{i-1}
$$

Thus the temperature error is

$$
\sigma_{T}^{2}=\frac{\sigma_{T_{B}}^{2} S^{2}\left(T_{B}\right)+\sigma_{V}^{2}+\sigma_{F}^{2}}{s^{2}(T)} .
$$

Table 5 lists the thermocouple Seebeck voltage $V$, fit uncertainty $\sigma_{F}$, Seebeck coefficient $d V / d T$ and temperature uncertainty $\sigma_{T}$ as a function of temperature over the temperature range $6 \mathrm{~K}<\mathrm{T}<280 \mathrm{~K}$. The bath temperatures were $4.2 \mathrm{~K}$ for $\mathrm{T}<80 \mathrm{~K}$ and $77.1 \mathrm{~K}$ for $\mathrm{T}>80 \mathrm{~K}$. The temperature uncertainty was large for $70 \mathrm{~K}<\mathrm{T}<80 \mathrm{~K}$ because the calibration in this temperature range was an extrapolation beyond the last data point taken at $72 \mathrm{~K}$. As seen in Table 5, the uncertainty in measuring temperature was less than $60 \mathrm{mK}$ over most of the temperature range.

The differential thermocouple was calibrated from $5.4 \mathrm{~K}$ to $79 \mathrm{~K}$ by measuring the "cold" thermometer temperature T, the temperature gradient, $\Delta T$ and the differential Seebeck EMF, $V_{\Delta T} \cdot 34$ data points were taken. 
Table 5. Uncertainty in absolute temperature measurement

\begin{tabular}{ccccc}
\hline $\begin{array}{c}\text { Temperature } \\
(\mathrm{K})\end{array}$ & $\begin{array}{c}\text { Seebeck } \\
\text { Voltage }(\mathrm{mV})\end{array}$ & $\begin{array}{c}\text { Uncertainty } \\
\text { Fit }(\mu \mathrm{V})\end{array}$ & $\begin{array}{c}\mathrm{dV} / \mathrm{dT} \\
(\mu \mathrm{V} / \mathrm{K})\end{array}$ & $\begin{array}{c}\text { Uncertainty } \\
\text { Temperature (mK) }\end{array}$ \\
\hline 6 & 0.0128 & 0.52 & 14.33 & 50 \\
10 & 0.0751 & 0.38 & 16.26 & 38 \\
13 & 0.1240 & 0.33 & 16.33 & 37 \\
14 & 0.1398 & 0.29 & 16.27 & 36 \\
20 & 0.2350 & 0.28 & 15.39 & 37 \\
30 & 0.3817 & 0.31 & 14.01 & 42 \\
40 & 0.5172 & 0.31 & 13.27 & 44 \\
60 & 0.7881 & 0.50 & 13.82 & 51 \\
70 & 0.9294 & 1.23 & 14.41 & 92 \\
76 & 1.0080 & 15.8 & 9.88 & 1605 \\
82 & 0.0670 & 0.76 & 14.44 & 63 \\
100 & 0.3500 & 0.75 & 16.36 & 55 \\
150 & 1.2104 & 0.64 & 18.23 & 45 \\
200 & 2.1538 & 0.84 & 19.47 & 50 \\
250 & 3.1583 & 0.73 & 20.46 & 43 \\
280 & 3.7784 & 1.8 & & 9 \\
\hline
\end{tabular}


The relative Seebeck coefficient of $A u-0,03 \% \mathrm{Fe}$ vs chromel $\left(V_{\Delta T} / \Delta T\right)$ was fitted to an eighth degree polynomial over two temperature ranges:

$5.4 \mathrm{~K}$ to $14.5 \mathrm{~K}$ and $14.5 \mathrm{~K}$ to $95 \mathrm{~K}$.

$$
S(T)=\frac{V_{\Delta T}}{\Delta T}=\sum_{i=0}^{8} B_{i} T^{i}
$$

The standard deviation of the fits were

$$
\begin{array}{llrl}
1.76 \times 10^{-2} \mu \mathrm{V} / \mathrm{K} & \text { for } & 5.4 \mathrm{~K}<\mathrm{T}<14.5 \mathrm{~K} \\
7.5 \times 10^{-3} \mu \mathrm{V} / \mathrm{K} & \text { for } & 14.5 \mathrm{~K}<T<79 \mathrm{~K}
\end{array}
$$

The uncertainty of the Seebeck coefficient fit for $5.4 \mathrm{~K}<\mathrm{T}<79 \mathrm{~K}$ is

$$
\underset{F}{\left(\sigma^{\prime}\right)^{2}}=\left(S_{V}^{\prime}\right)^{2} \sum_{i=0}^{8} \quad \sum_{j} \sum_{0} \cdot \varepsilon_{i j}^{i} T^{i+j} .
$$

$S_{V}^{i}$ is the varience of the differentlal thermocouple $f_{i t}$ and $\varepsilon_{i j}^{\prime}$ is the error matrix.

Above $80 \mathrm{~K}$ the Seebeck coefficient was calculated by differentiating equation 86 with respect to $T$

$$
S(T)=\frac{d V}{d T}=\sum_{i-1}^{9} \quad i A_{i} T^{i-1}
$$

The uncertainty in calculating $S(T)$ for $T>80 \mathrm{~K}$ is

$$
\left(\begin{array}{c}
\sigma_{1} \\
F
\end{array}\right)^{2}=s_{V}^{2} \sum_{i=1}^{9} \sum_{j=1}^{9} \varepsilon_{i j} i j\left[T^{i+j-2}\right]
$$


The temperature gradient was measured by measuring the "cold" thermometer probe temperature $r$ and the differential Seebeck yoltage $V_{\Delta T}$. The temperature gradient was

$$
\Delta T=\frac{V_{\Delta T}}{S(T+1 / 2 \Delta T)} .
$$

The uncertainty in the measurement of $\Delta T$ was (neglecting the uncertainty in measuring $T$ )

$$
\sigma_{\Delta T}^{2}=\Delta T^{2} \frac{\sigma_{V T}^{2}}{v^{2}{ }_{\Delta T}^{2}}+\frac{\left(\sigma_{F}^{\prime}\right)^{2}}{S\left(T+\frac{1}{1 / 2 \Delta T)}\right.}
$$

where the uncertainty in measuring $V_{\Delta T}$ was, $\sigma_{V_{\Delta T}}=0.005 \mu \mathrm{V}$.

The Seebeck coefficient, Seebeck coefficient uncertainty and temperature gradient uncertainty are listed in Table 6 for several values of temperature gradient. The uncertainty in the temperature gradient was large for temperatures near $80 \mathrm{~K}$ and $280 \mathrm{~K}$. The large uncertainty was due to the difficulty in fitting the data near the end points of tlie fit. Above $80 \mathrm{~K}$, the Seebeck coefficient was calculated from the fit of the absolute thermocouple. Consequently a systematic error may exist in the temperature gradient measurement above $80 \mathrm{~K}$ if the differential thermocouple had a different Seebeck coefficient than the absolute thermocouple. Comparison of Tables 5 and 6 show that the difference between the measured Seebeck coefficient of the differential thermocouple and the derivative of the absolute thermocouple fit at $70 \mathrm{~K}$ was $1.8 \%$. At $6 \mathrm{~K}$ 
Table 5. Uncertainty in the thermocouple Seebeck coefficient and temperature gradient measurement

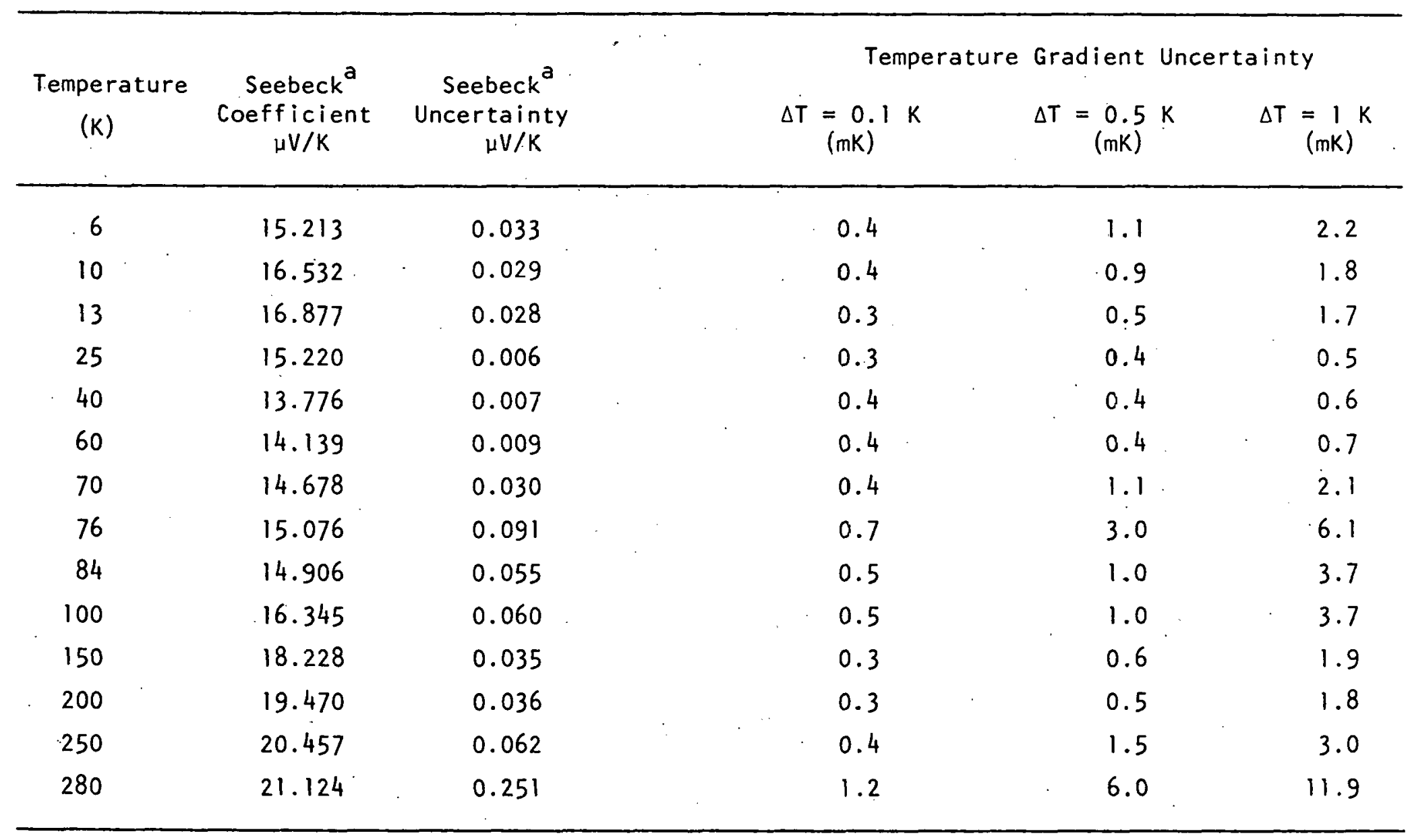

above $76 \mathrm{~K} S(T)$ was calculated from $\mathrm{dV} / \mathrm{dT}$ of the absolute thermocouple. 
the difference was as large as $5,8 \%$. Thus the Seebeck coefficient of the two thermocouples were different, However the differences decreased as the temperature was. increased, At $80 \mathrm{~K}$ it was difficult to accurately $f i t$ the absolute thermocouple such that the derivative of the fit matched the measured Seebeck coefficient data taken at lower temperatures, Consequently, as seen in Table 8 , at $84 \mathrm{~K}$ the calculated Seebeck coefficient was less than the measured Seebeck coefficient at $76 \mathrm{~K}$. Thus the actual error in the thermocouple measurement was much larger than the fitting error shown in Table 8 for $80 \mathrm{~K}<\mathrm{T}<100 \mathrm{~K}$. 